\title{
DISCLAIMER
}

\begin{abstract}
This report was prepared as an account of work sponsored by an agency of the United States Government. Neither the United States Government nor any agency thereof, nor any of their employees, makes any warranty, express or implied, or assumes any legal liability or responsibility for the accuracy, completeness, or usefulness of any information, apparatus, product, or process disclosed, or represents that its use would not infringe privately owned rights. Reference herein to any specific commercial product, process, or service by trade name, trademark, manufacturer, or otherwise does not necessarily constitute or imply its endorsement, recommendation, or favoring by the United States Government or any agency thereof. The views and opinions of authors expressed herein do not necessarily state or reflect those of the United States Government or any agency thereof.
\end{abstract}

\section{Search for Right-Handed Currents in Muon Decay}

\author{
Alexander Ezra Jodidio \\ Ph.D. Thesis \\ January 1986 \\ Lawrence Berkeley Laboratory \\ University of California \\ Berkeley, CA 94720
}

This work was supported by the Director, Office of Energy Research, Office of High Energy and Nuclear Physics, Division of High Energy Physics of the U. S. Department of Energy under contract \#DE-AC0376 SF00098. 


\section{Search for Right-Handed Currents \\ in Muon Decay}

Alexander Ezra Jodidio

Abstract

Limits are reported on charged right-handed currents, based on precise measurement of the endpoint $e^{+}$spectrum $1{ }^{\prime 2} \mu^{+}$decay. Highly polarized $\mu^{+}$from the TRIUMF "surface" muon beam were stopped in high purity metal foils and liquid He targets selected to minimize depolarization effects. Decay $\mathrm{e}^{+}$emitted within 160 mrad of the beam direction were momentum-analyzed to $0.15 \%$. Muons were stopped within either a spin-precessing transverse field (70-G or 110-G) or a spin-holding longitudinal field $(0.3-\mathrm{T}$ or $1.1-\mathrm{T})$. Data collected with the spin-precessing field were used for the momentum calibration of the spectrometer. The spin-held data were used to measure the relative $e^{+}$rate at the endpoint. An extrapolation was made to extract the endpoint rate opposite to the $\mu^{+}$spin. In terms of the standard muon decay parameters this rate is given by $\left(1-E P_{\mu} \delta / \rho\right)$ where $P_{\mu}$ is muon polarization. The result for $\xi P_{\mu} \delta / p$ was consistent with the $\mathrm{V}-\mathrm{A}$ prediction of 1 . It is quoted as a $90 \%$ confidence lower limit $\xi P_{\mu} \delta / \rho>0.9975$ since we are unable to correct for all possible sources of muon depolarization. For the model with manifest left-right symmetry and massless neutrinos the result implies $90 \%$ 
confidence limits $m\left(W_{2}\right)>432 \mathrm{GeV} / \mathrm{c}^{2}$ and $-0.050<\zeta<0.035$, where $\mathrm{W}_{2}$ is the predominantly right-handed boson and $\zeta$ is the left-right mixing arigle. With the assumption of no left-right mixing an equivalent $90 \%$ confiderce upper limit of 0.025 is obtained on the absolute value of the ratio of a possible $\mathrm{V}+\mathrm{A}$ amplitude to the dominant $\mathrm{V}-\mathrm{A}$ amplitude in muon and pion decays. Limits are also deduced on the $v_{\mu L}$ mass and helicity in $\pi^{+}$decay, non-(V-A) couplings in helicity projection form, the mass scale of composite leptons, and the branching ratio for $\mu \rightarrow e+f$ where $f$ (familon) is the neutral massless Nambu-Goldstone boson associated with flavor symmetry breaking. 
Table of Contents

Acknowledgements

I. Introduction $\cdot \quad \cdot \quad \cdot \quad \cdot \quad \cdot \quad \cdot \quad \cdot \quad \cdot \quad \cdot \quad \cdot 1$

II. Mion Decay Spectrum • • • • • • • • 6

III. Experimental Setup . . . • • . • 13

A. Overview.$\quad \cdot \quad \cdot \quad \cdot \quad \cdot \quad \cdot \quad \cdot 13$

B. The Beamline . . . . . . . . 16

C. Muon Deceleration and Thermalization . . 21

D. The Muon Polarimeter . . . . . 24

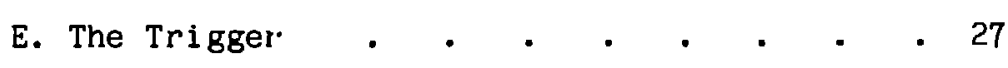

IV. Event Reconstruction and Selection . . . . 29

V. Data Analysis . • . • • • • • 33

A. The Fitting Procedure . . . . . . 34

B. Computation of $\cos \theta_{\text {ay }} \quad \cdot \quad \cdot \quad \cdot \quad \cdot \quad \cdot 39$

C. Summary of the Analysis Method • . - 44

D. Data Fitting Results - . • • • 46 
VI

Corrections • • . . . . . . . . 53

A. Correction due to Multiple Scattering and Finite Chamber Resolution . . . . 55

B. Correction due to Use of Data-Averaged $\cos \theta_{\mu} \cos \theta_{\mathrm{e}} \quad \cdot \quad \cdot \quad \cdot \quad \cdot \quad \cdot \quad \cdot \quad \cdot \quad \cdot 62$

C. Muon Depolarization in Scattering with Unpolarized Electrons . . . . . . 64

VII. Systematic Errors . . . . . . . . 67
A. $x=1$ Calibration
67
B. Momentum Acceptance
70
C. Momentum Dispersion
72
D. Reconstruction of $\cos \theta_{\mu}$ and $\cos \theta_{e} \cdot$. $\quad 73$
E. Extra Muons • • • • • • •

VIII. Discussion and Conclusions . . . . . 78

A. Limit on $\xi \mathrm{P}_{\mu} \delta / \rho$. $\quad . \quad$. $\quad . \quad$ - 78

B. Limits on Right-Handed Currents with Massless Neutrinos . . . . . • 79

C. Limits on $m\left(v_{\mu L}\right)$ and $v_{\mu L}$ Helicity

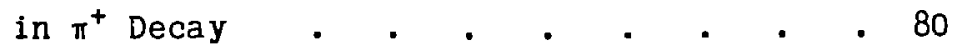

D. Restriction on the Lorentz Structure of Leptonic Charged Weak Interactions - . 80 E. Limits on Composite Leptons . . . 83 F. Limils on Familons . • . . . . 85 
G. Limits on $m\left(W_{2}\right)$ with $m\left(\nu_{\mu R}\right) \neq 0$. . . . 86

H. Concluding Remarks . . . . . 89

Appendix A. Stopping Targets . . . . . 90

Appendlx B. Table of Data Fitting Results - . 94

Appendix C. Computation of Limits on $m\left(W_{2}\right)$

with $m\left(v_{\mu R}\right) \neq 0 \quad$ - $\quad . \quad$. $\quad .97$

References . . . . . . . . . 103 
Acknowledgements

This thesis is based on the results from experiment 185 performed at the TRIUMF meson facility by a collaboration from LBL/University of California at Berkeley, Northwestern University, and TRIUMF. The success of the experiment was made possible by the efforts of all collaborators: Brian Balke, John Carr, George Gidal, Bruno Gobbi, Chris Oram, Kirk Shinsky (deceased), Herb Steiner, David Stoker, Mark Strovink (spokesman), Bob Tripp, and myself. The technical assistance of C. Covey, R. Fuzesy, F. Goozen, P. Harding, M. Morrison, and P. Robrish at LBL and the support of the TRIUMF staff contributed to the success of the experiment.

I would like to expess my sincere appreciation to my advisor, Professor H.M. Steiner, for his strong support throughout my work on this experiment.

I wish to thank the members of my thesis committee, professors H.M. Steiner, M. Strovink and S. Kaplan for making many helpful comments on this thesis. Thanks are also due to my fellow graduate student Brian Balke for many helpful suggestions on chapters VI and VII.

I would also like to thank professor Bruno Gobbi of Northwestern University for his hespitality and his assistance during the analysis of the data at Evanston in the summer of 1983.

I cannot say enough to express my gratitude to my wife, Anna Rozenshtein, for her encouragement and moral support. Without her this thesis may not have been completed. 
$I$, and I am sure all other members of the collaboration, have sorely missed the presence of Kirk Shinsky, who was tragically stricken by cancer and passed away on September 6, 1983. We deeply regret that he did not see the final results from this experiment, to which he made such a strong contribution.

The research reported here was supported in part by the U.S. Department of Energy through Contracts No. DE-AC03-76SF00098 and AC02-ER02289. 


\section{INTRODUCTION}

In the widely accepted Glashow-Weinberg-Salam (GWS) SU(2) $\mathrm{L} X \mathrm{U}(1$ ) model of weak and electromagnetic interactions ${ }^{1}$ the $V-A$ structure of weak interaction has been put in a friori to obtain agreement with experiments. Although the model agret: with all experimental results up to presently available energies, the experimental precision has not been sufficient to rule out relatively large deviations from the V-A structure. ${ }^{2}$ We have made a sensitive search for such deviations by measuring the muon decay spectrum near the end-point. Our result is particularly suited for setting stringent limits on charged right-handed, i.e. $V+A$, currents, and for constraining parameters in the left-right symmetric models.

The left-right symmetric (LRS) models, ${ }^{3}$ based on the gauge group $\mathrm{SU}(2)_{\mathrm{R}} \times \mathrm{SU}(2)_{\mathrm{L}} \times \mathrm{U}(1)$, have been intensively studied as extensions of the standard GWS model. Such modeis have a great aesthetic appeal because the Lagrangian has a $L-R$ symmetry and parity conservation is restored at moderate energies. In such models the $V-h$ nature of weak interaction at low energies is due to the spontaneous symmetry breaking mechanism, as a result of which the predominantly $r i g h t-h a n d e d$ gauge boson $W_{2}$ acquires a heavier mass than the predominantly left-handed boson $W_{1} \cdot{ }^{4}$ The mass-squared ratio or physical bosons $W_{1}$ and $W_{2}$ will be denoted by $\varepsilon=m^{2}\left(W_{1}\right) / m^{2}\left(W_{2}\right)$. The mass eigenstates $W_{1}$ and $W_{2}$ are related to $W_{L}$ and $W_{R}$ by a mixing angle $\zeta, \mathrm{W}_{1}=\mathrm{W}_{\mathrm{L}} \cos \zeta-\mathrm{W}_{\mathrm{R}} \sin \zeta, \mathrm{W}_{2}=\mathrm{W}_{\mathrm{L}} \sin \zeta+\mathrm{W}_{\mathrm{R}} \cos \zeta$.

Let us review the existing linits on charged right-handed 
currents. The limits that can be obtained from leptonic and semi-leptonic weak processes depend on the masses of the associated right-handed neutrinos. If neutrinos are Dirac fermions, $v_{L}$ and $v_{R}$ must necessarily have the same mass, since they are different helicity states of the same particle. In this case the predominantly rlght-handed boson $W_{2}$ participates in low-energy processes. Hciever, in some attractive theories neutrinos are Majorana particles. 5 Since there can be both Majorana and Dirac mass terms in the neutrino mass matrix, the physical neutrinos $v_{1}$ and $v_{2}$ may have different masses. In most models the predominantly left-handed neutrino $v_{1}$ is predicted to be very light, $m\left(v_{1}\right) \sim m_{e}^{2 / m}\left(w_{2}\right)$, while the predominantly $r i g h t-h a n d e d ~ n e u t r i n o ~ v_{2}$ is very hezvy, $m\left(v_{2}\right) \sim m\left(W_{2}\right)$, and therefore cannot be produced in low energy experiments. The physical neutrinos $\nu_{1}$ and $\nu_{2}$ are related to $\nu_{L}$ and $\nu_{R}$ by a mixing angle $\delta$, expected to be of the order of $\mathrm{m}_{\mathrm{e}} / \mathrm{m}\left(\mathrm{W}_{2}\right)$. Ignoring the very small neutrino mixing angle, purely leptoni: processes, requiring production of at least one $v_{2}$, do not set limits on right-handed currents in such theories. Semi-leptonic processes such as $\nu N$ and $\overline{\nu N}$ scattering, which do not require a production of $v_{2}$, can still set a limit on the mixing angle $\zeta$.

Hadronic weak processes set limits on right-handed currents independently of $v_{R}$ masses. In a class of models, called 'manifestly' L-R symmetric, the left-handed and right-handed Kobayashi-Maskawa quark mixing angles are assumed to be identical, and $C P$ invariance is assumed to hold. In these models the $K_{L}-K_{S}$ mass difference requires $m\left(W_{2}\right)>1.6 \mathrm{TeV},{ }^{6}$ and current algebra analysis of 
$\Delta S=1$ decays yields $\zeta 50.004, \mathrm{~m}\left(W_{2}\right)>300 \mathrm{GeV}$ for $\zeta=0 .{ }^{7}$ If left-handed and right-handed mixing angles are not 1 dentlcal (winich is a very unnatural and unaesthetic assumption), hadronic processes are consistent with $\mathrm{m}\left(\mathrm{W}_{2}\right)-300 \mathrm{GeV} .^{8}$

Another strong limit on the mixing angle $\zeta, \zeta<0.005$, has been obtained in a model-dependent analysis of semileptonic weak processes, assuming again manifest L-R symmetry. ${ }^{9}$

The contours corresponding to $90 \%$ confidence limits ${ }^{10}$ on $\varepsilon$ and $\zeta$ from the experiments in $\beta$ decay, $\mu$ decay and $v N, \overline{v N}$ scattering, are shown in Fig. I.1. The allowed regions contain the origin $\varepsilon=\zeta=0$, which is the V-A limit. Manifest L-R symmetry has been assumed. The contours from $\mu$ and $\beta$ decay experiments have been plotted with the assumption that the right-handed neutrinos are sufficiently light not to affect the kinematics. The bold ellipse in Fig. I.1 is the combined result from the analysis of muon decay spectrum at the end-point, opposite the $\mu^{+}$spin, presented in this thesis, and from the $\mu$ SR analysis published previously.11,12 The other muon-decay contours are derived from the measurement of the polarization parameter $\xi P_{\mu}$ (dotted curve, Ref. 13) and the Michel parameter $p$ (solid curve, Ref. 14). Nuclear B decay contours are derived from the Gamow-Teller B polarization (dot-dashed curve, Ref. 15); the comparison of Gamow-Teller and Fermi B polarizations (long-dashed curves, Ref. 16); and the ${ }^{19^{9}} \mathrm{Ne}$ asymmetry $\mathrm{A}(0)$ and ft ratio, with the assumption of conserved vector current (short-dashed curves, Ref. 17). The limits from the $y$ distributions in $v N, \overline{v N}$ scattering (double Iines, Ref. 18) are valid irrespective of $v_{\mathrm{R}}$ mass. 


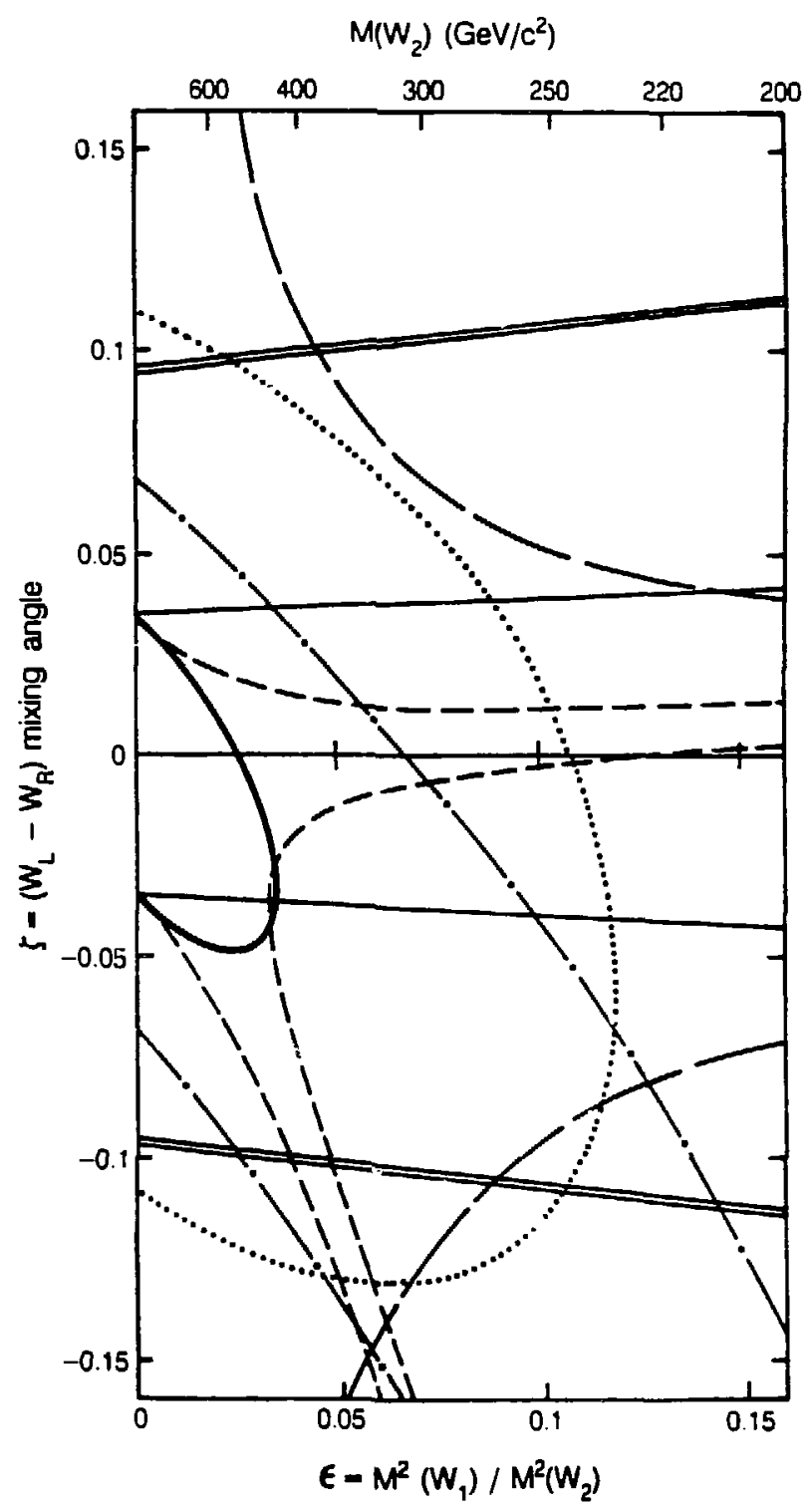

XBL $8512 \cdot 11762$

FIG. I.1. Experimental 90\%-confidence limits on the mass-squared ratio $\varepsilon$ and mixirg angle $\zeta$ for the gauge bosons $W_{1}$ and $W_{2}$. The allowed regions are those which include $\varepsilon=\zeta=0$. The sources of the limits are described in the text. 
The rest of this thesis is organized as follows. In section II we discuss the properties of the muon decay spectrum and present the basic idea in the data analysis. The beamline and experimental apparatus are discussed in section III. Event reconstruction and selection are considered in section IV. Data analysis and data fitting results are presented in section $V$. Corrections and systematic errors are discussed ir, sections VI and VII. The conclusions from the experimental result are drawn in section VIII. 


\section{MUON DECAY SPECTRUM}

In the discussion of the muon decay spectrum we will assume that neutrinos are sufficiently light not to affect the kinematics. We will return to the question of massive neutrinos in section VIII.

The muon differential decay rate for an interaction mediated by a heavy vector boson $W$ differs from the decay rate computed with the corresponding four-fermion contact interaction Hamiltonian by terms ${ }^{19}$ of order $\left(m_{\mu} / M_{W}\right)^{2}$. These terms are $-10^{-6}$ for $M_{W}=80 \mathrm{GeV} / \mathrm{c}^{2}$ and are negligible at the present level of experimental precision. Consequently we will use the expression for the muon decay spectrum computed for a four-fermion contact interaction.

If radiative corrections are ignored, the muon differential decay rate, ${ }^{20}$ integrated over $\mathrm{e}^{+}$spin directions, is given by

$$
\begin{aligned}
\frac{d^{2} \Gamma}{x^{2} d x d(\cos \theta)}- & {\left[(3-2 x)+\left(\frac{4}{3} \rho-1\right)(4 x-3)+12 \frac{m_{e}}{m_{\mu}} \frac{x-1}{x} n\right] } \\
& -\left[(2 x-1)+\left(\frac{4}{3} \delta-1\right)(4 x-3)\right] \xi P_{\mu} \cos \theta .
\end{aligned}
$$

Here $x$ is the standard reduced energy variable $x=E_{e} / E_{\max }$, where $E_{\max }=\left(m_{e}{ }^{2}+m_{\mu}{ }^{2}\right) /\left(2 m_{\mu}\right)=52.83 \mathrm{MeV}$ is the maximum energy; $m_{e}$ and $m_{\mu}$ are the particle masses. The effects of finite positron mass are neglected in the above formula but not in the analysis. $\pi^{-\theta}$ is the angle between positron momentum and muon polarization vector $P_{\mu}$ in the $\mu^{+}$rest frame. The four muon decay parameters $\rho, n, \xi, \delta$ have been previously measured, and their world average values 21 prior to our experiment are given in Table II.1. The values these parameters take in the L-R symmetric model are also listed in Table II. 1 to 
TABLE II.1. Values of the muon decay parameters $\rho, \eta, \xi, \delta$ in the $V-A$ model and the manifestly left-right symmetric (LRS) model with massless neutrinos. Their world-average experimental values ${ }^{21}$ prior to our experiment are also listed. The values in the LRS model are given to the lowest order in the mass-squared ratio $\varepsilon$ and mixing angle $\zeta$ for the gauge bosons $W_{1}$ and $W_{2}$.

\begin{tabular}{|c|c|c|c|}
\hline $\begin{array}{c}\text { Decay } \\
\text { Parameter }\end{array}$ & $\begin{array}{c}V-A \\
\text { Value }\end{array}$ & $\begin{array}{l}\text { Value in } \\
\text { in the LRS model }\end{array}$ & Experimental Value \\
\hline$\rho$ & $\frac{3}{4}$ & $\frac{3}{4}\left(1-2 \zeta^{2}\right)$ & $0.7517 \pm 0.0026$ \\
\hline$n$ & 0 & 0 & $0.06 \pm 0.15$ \\
\hline $\begin{array}{l}\xi \\
P_{\mu}^{*}\end{array}$ & $\begin{array}{l}1 \\
1\end{array}$ & $\left.\begin{array}{l}1-2 \varepsilon^{2}-2 \zeta^{2} \\
1-2(\varepsilon+\zeta)^{2}\end{array}\right\}$ & $\xi P_{\mu}: 0.972 \pm 0.014$ \\
\hline$\delta$ & $\frac{3}{4}$ & $\frac{3}{4}$ & $0.7551 \pm 0.0085$ \\
\hline
\end{tabular}

* $P_{\mu}$ is the muon longitudinal polarization from $\pi^{+}$decay at rest. 
Specification of $\theta_{l}, \theta_{F}, \phi_{l}$, and $\phi_{F}$ for the two-layer design permits one to seek values of $\theta_{2}, \theta_{3}, \theta_{4}$, and $\phi_{2}$ such that harmonic components of orders $m=3,5,7$, and 9 simultaneously vanish. The requisite conditions for this design then become (neglecting, for simplicity, the contributions from image fields):

$\frac{\sin m \theta_{1}-\sin m \theta_{2}+\sin m \theta_{3}-\sin m \theta_{4}+\sin m \theta_{F}}{R_{A}^{m-1}}$
$+\frac{\sin m \phi_{1}-\sin m \phi_{2}+\sin m \phi_{F}}{R_{B}^{m-1}}=0$

for $m=3,5,7$, and 9 . Solutions are conveniently sought computationally by again employing the IMSL Routine ZSYSTM (as in the VAX program listed by V.O. Brady as SUPER.FOR;28).

\section{Results}

(i) Sinqle-Layer, Two-Block Design:

With $\theta_{F}$ assigned successively various values $\geqslant 45^{\circ}$, the corresponding values for $\theta_{1}$ and $\theta_{2}$ which simultaneously depress harmonic components of orders $m=3$ and $m=5$ are as shown in Table I and on Fig. 1 . It is noted that for $\theta_{F}=45^{\circ}$ the widths of the individual blocks vanish $\left(\theta_{1} \rightarrow 0\right.$ and $\left.\theta_{2} \rightarrow \theta_{F}\right)$. For values of $\theta_{F}$ in the neighborhood of $64^{\circ}$ the requisite wedge thickness $\theta_{2}-\theta_{1}$ is seen to pass through a minimum, and for a slightly larger value $\left(\theta_{F} \cong 67.275^{\circ}\right)$ the harmonic component of order $m=7$ is found to vanish. 


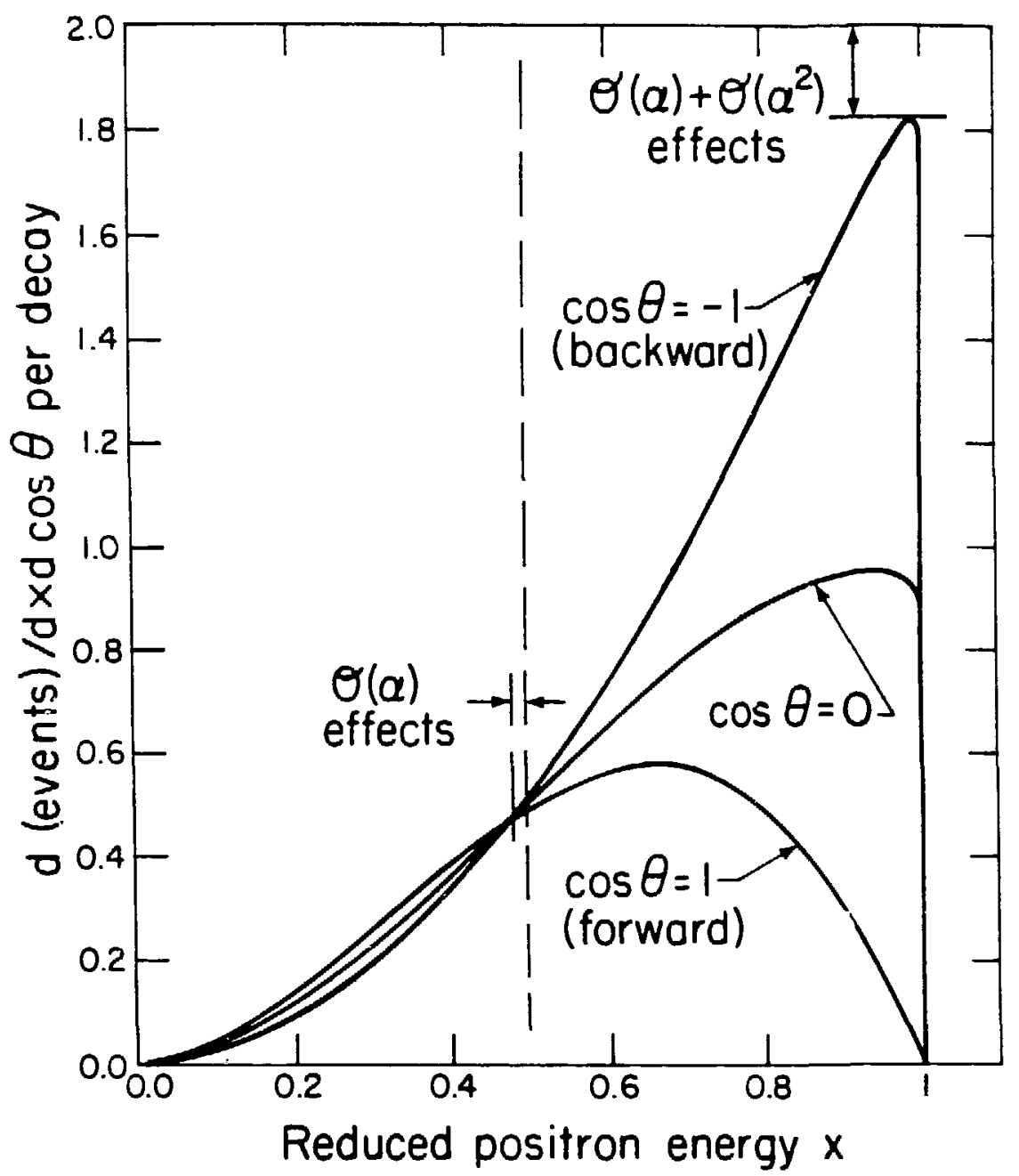

XBL $8512 \cdot 11756$

FIG. II.1. The V-A radiatively corrected muon decay spectrum plotted for $\cos \theta=+1,0$ and -1 . The effects of radiative corrections are also indicated. 
small admixture of $V+A$, scalar, tensor or pseudoscalar currents, then the positron spectrum near the endpoint, for $\cos \theta=1$, can be expressed as a sum of two pure $V-A$ spectra: one with $P_{\mu}=\cos \theta=1$ and another with $P_{\mu} \cos \theta=0$. An intuitive explanation is that near the end-point a positron spectrum at a particular value of $\cos \theta$ is given essentially by a sum of a linear function characteristic of the $P_{\mu} \cos \theta=1$ V-A spectrum, which vanishes at $x=1$, and a step function, represented by the unpolarized, $P_{\mu} \cos \theta=0, V-A$ spectrum. The relative size of the step or the rate at the endpoint is $1-\xi \mathrm{P}_{\mu} \cos \theta \delta / \rho$. Measurement of the rate as a function of $\cos \theta$ allows us to extract $\xi \mathrm{P}_{\mu} \delta / \rho$.

In order to simplify the algebra it will te convenient to use

$$
\begin{aligned}
& \tilde{\rho}=1-\frac{4}{3} \rho \\
& \tilde{\delta}=1-\frac{4}{3} \delta
\end{aligned}
$$

with $\tilde{\rho}=\tilde{\delta}=0$ for a pure $V-A$ interaction. The decay spectra will be denoted by

$$
\begin{aligned}
S\left(x, P_{\mu} \cos \theta\right)- & \text { spectrum for an arbitrary weak coupling } \\
& \text { (i.e. arbitrary values of } \xi, \rho, \delta) \text { and a } \\
& \text { particular value of } P_{\mu} \cos \theta \\
S_{V-A}(x, 1) \quad- & V-A \text { spectrum at } P_{\mu} \cos \theta=1 \text { with the same normal- } \\
& \text { ization as } S\left(x, P_{\mu} \cos \theta\right) \\
S_{V-A}(x, 0) \quad- & V-A \text { spectrum at } P_{\mu} \cos \theta=0 \text { with the same normal- } \\
& \text { ization as } S\left(x, P_{\mu} \cos \theta\right) .
\end{aligned}
$$

Let us ignore for the moment the radiative corrections. Since near the endpoint the $\eta$ term in (2.1) is negligible and can be ignored, there is an exact relationship

$$
S\left(x, P_{\mu} \cos \theta\right)=q(\theta) S_{V-A}(x, 1)+r(\theta) S_{V-A}(x, 0)
$$


where

$$
q(\theta)=\xi P_{\mu} \cos \theta-\frac{1}{2} \tilde{\delta} \xi P_{\mu} \cos \theta+\frac{1}{2} \tilde{\rho}
$$

and $r(\theta)$ is the relative rate at the end-point

$$
r(\theta)=\left(1-\xi P_{\mu} \cos \theta\right)+\tilde{\delta} \xi P_{\mu} \cos \theta-\tilde{\rho}
$$

Equations (2.3) and (2.4) can be rewritten in a simpler form using the smallness of $\tilde{\rho}$ and $\tilde{\delta}(\sim .0 i)$, and the fact that $\xi \mathrm{P}_{\mu} \cos \theta$ is approximately 1. To first order in $\tilde{\rho}, \tilde{\delta}, r(\theta)$ and $q(\theta)$ take the simple forms

$$
r(\theta)=i-\xi P_{\mu} \frac{\delta}{p} \cos \theta
$$

and

$$
\mathrm{q}(\theta)=1-\mathrm{r}(\theta)=\xi \mathrm{P}_{\mu}{ }_{\rho}^{\delta} \cos \theta
$$

When radiative correctluns are inclured, the exact relation (2.2) becomes only an approximation, and if the data were fitted according to (2.2), with $q(\theta)=1-r(\theta)$, then $r(\theta)$ would only approximately correspond to (2.5). The high precision of this measurement has required a detailed study of the effect of the radiative corrections. For the fitting procedure to be described in section $v$, It was checked, that for a spectrum given by a combination of only $\mathrm{V}+\mathrm{A}$ and $\mathrm{V}-\mathrm{A}$ effective couplings, the radiative corrections had a very small effect on the value found for $r(\theta)$. Hence, within the experimental orrors, $E P_{\mu} \delta / \rho$ could be extracted from $r(\theta)$ as in (2.5) without introcucing an additional systematic error. If our result is to be used for satting limits on the presence of other effective weak couplings, it must be verified that when all couplings are taken into account, the radiative corrections do not introduce an additional 
systematic error.

The highly polarized muons in our experiment were obtained by means of a "surface" muon beam, ${ }^{25}$ derived from pions decaying at rest near a surface of the production target. Right-handed currents would reduce muon polarization in pion decay to $P_{\mu}=1-2(\varepsilon+\zeta)^{2}$ (assuming manifest left-right symmetry). When this effect is included, our final result is given in terms of $\varepsilon$ and $\zeta$ by

$$
\xi P_{\mu} \delta / \rho=1-2\left(2 \varepsilon^{2}+2 \varepsilon \zeta+\zeta^{2}\right)
$$

Since we are unable to correct for all possible sources of muon depolarization, our result can be interpreted only as a lower limit on $\xi P_{\mu} \delta / \rho$. 


\section{EXPERIMENTAL METHOD}

\section{III.A. Overview}

The experiment was made possible by the nearly complete polarization of the "surface" muon beam, derived from pions decaying at rest near the surface of the production target. The "surface" muons were transported in vacuum by the $M 13$ beamline at the TRIUMF cyclotron to a stopping target in the muon polarimeter, shown schematically in Fig. III.1. High-purity metal foils (Ag, Al, Au, $\mathrm{Cu}$ ) and $\mathrm{liquid}$ He were selected as stopping targets, since in these materials muonium $\left(\mu^{+}-\mathrm{e}^{-}\right.$atom $)$formation, leading to muon depolarization, is strongly suppressed.

The target region was immersed either in a strong longitudinal 'spin-holding' fleld (0.3-T or 1.1-T) aligned with the nominal beam direction, or a vertical (70-G or 110-G) spin-precessing field. The longitudinal field quenched muon depolarization in muonium via the Paschen-Back effect. The data collected with the longitudinal fleld were used to measure the rate at the spectrum endpoint. The data collected with the spin-precessing field, after the appropriate cuts on muon decay time, were equivalent to the unpolarized, i.e. $P_{\mu}=0$, data. In this analysis these data were used for the momentum calibration of the speatrometer.

The angular acceptance for positrons was significantly increased by the downstream portion of the solenold, which served as a $0.5 \mathrm{~T}-\mathrm{m}$ solenoidal field lens. The solenold lens fucused the decay positrons 


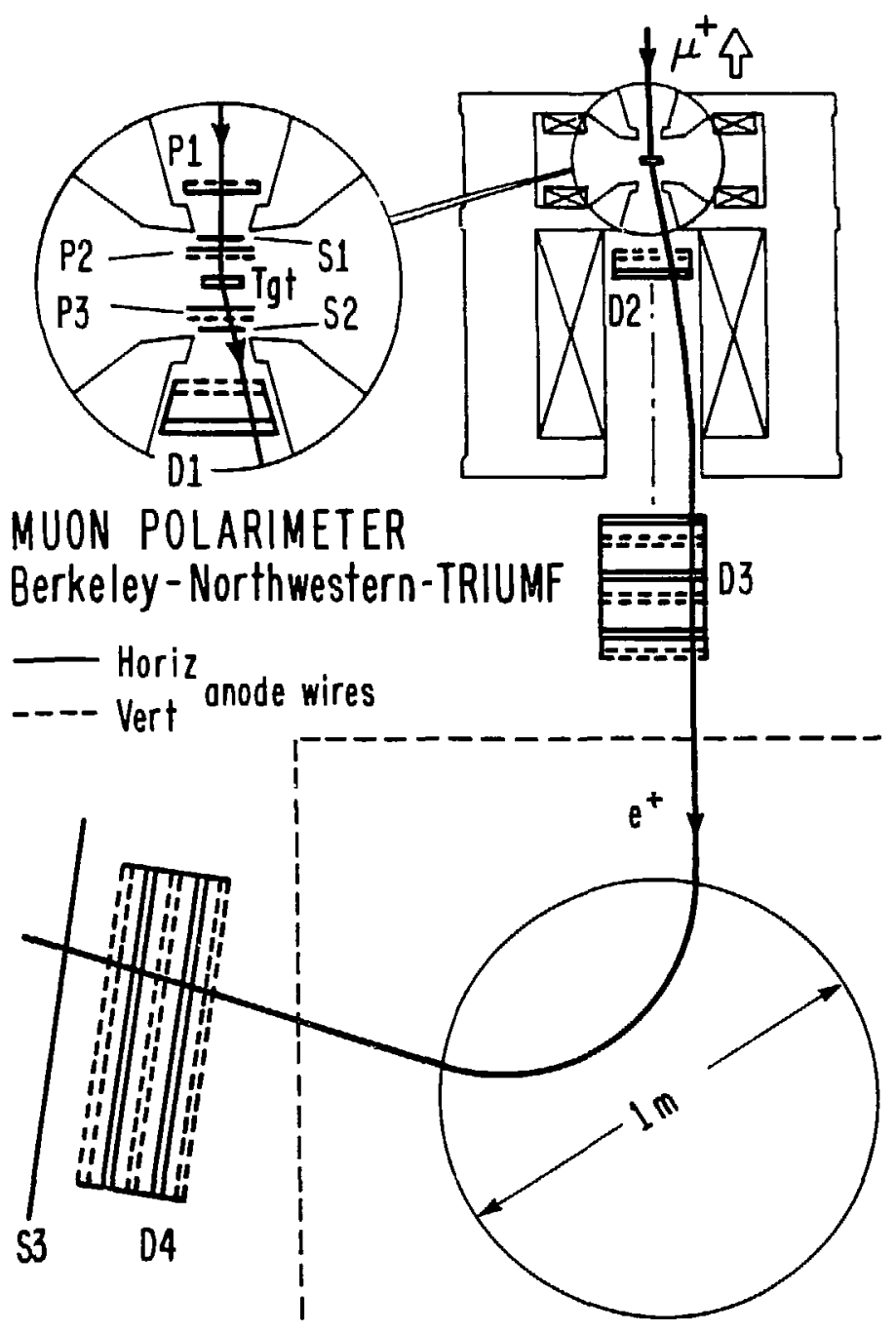

XBL 834-147

FIG. III.1. Plan view of muon polarimeter. P1-P3 are proportional chambers; S1-S3 are scintillators; D1-D4 are driftchambers. The veto scintillators $V 1$ and $V 2$ surrounding $S 1$ and S2 respectively are not shown. Muons entering the solenoid are stopped in the target ( $T$ gt). Decay $e^{+}$emitted near the beam direction are focused by the solenold onto the spectrometer. 
onto the dipole magnet spectrometer. The septum between the target and solenoid bore made the focal length of the solenoid lens nearly independent from the choice of target field orientation.

The horizontally-focusing spectrometer was chosen in order to achieve a high momentum resolution and to maintain a large angular acceptance for positrons in combination with the solenoid. The volume between the spectrometer conjugate focal planes was evacuated. The vacuum windows were positioned close to the focal planes to minimize the adverse effects of Coulomb scattering on momentum resolution.

Particle trajectories in the target region (see inset of Fig. III.1) were measured by the proportional chambers P1 and P2 for muons, and by the proportional for positrons. Positron trajectories outside the spectrometer focal planes were measured by the driftchambers D3 and D4. The scintillation counters S1, S2 and S3 provided trigger signals.

The data were accumulated in three running periods (to be denoted by Run 1 , etc.) at the TRIUMF cyclotron during 1982-84. The experimental conditions were essentially the same for all three running periods, except for minor differences mentioned below and in Appendix A. The longitudinal field in the stopping target region was 1.1-T for Runs 1 and 2, and $0.3-\mathrm{T}$ and $1.1-\mathrm{T}$ for Run 3. A total of $1.8 \cdot 10^{7}\left(1.4 \cdot 10^{7}\right)$ triggers were collected in the spin-holding (spin-precessing) mode on 130 (170) computer tapes (1600 BPI). Under the optimal conditions these data would have been accumulated in -20 continuos days of running time. In each of the three running periods 
the data were also collected in many special runs to be described in section IV. The data collected in these special runs were used for the momentum calibration of the spectrometer.

The "surface" muon beam, muon polarimeter and the trigger will be described in greater detail below. We will also briefly review the process of muon deceleration to thermal energies and the suppression of muon depolarization in muonium by the longitudinal field.

\section{III.B. The Beamline}

The experiment utilized the $M 13$ beamline ${ }^{26}$ at the TRIUMF meson facility. M13 is a low energy (20-130 MeV/c) muon and pion channel, which views a $2 \mathrm{~mm}$ or a $10 \mathrm{~mm}$ thick carbon production target at $135^{\circ}$ with respect to the primary proton beam (see Fig. III.2). The particles are transporied in vacuum through two $60^{\circ}$-bending dipoles, with momentum-selecting slits at the two foci allowing a momentum bite $\Delta p / p$ as low as $0.5 \%$. The beamline was tuned to deliver about $15,000 \mu^{+}$per second in a $1 \%$ momentum interval at a typical proton beam current of $100 \mu \mathrm{A}$. The particles were focused in a narrow $0.3 \mathrm{msr}$ cone on a $-1 \mathrm{~cm}$ radius spot at the muon stopping target.

Positive particle fluxes in the beamline, obtained in beam tuning studies of Ref. 26, are shown in Fig. III.3. Just below $29.8 \mathrm{MeV} / \mathrm{c}$ the muon flux is dominated by "surface" muons, produced by pions decaying at rest near the surface of the production target. In the experiment the beamline was tuned to $29.5 \mathrm{MeV} / \mathrm{c}$, just below the surface muon edge. At this beamline setting the surface muons 


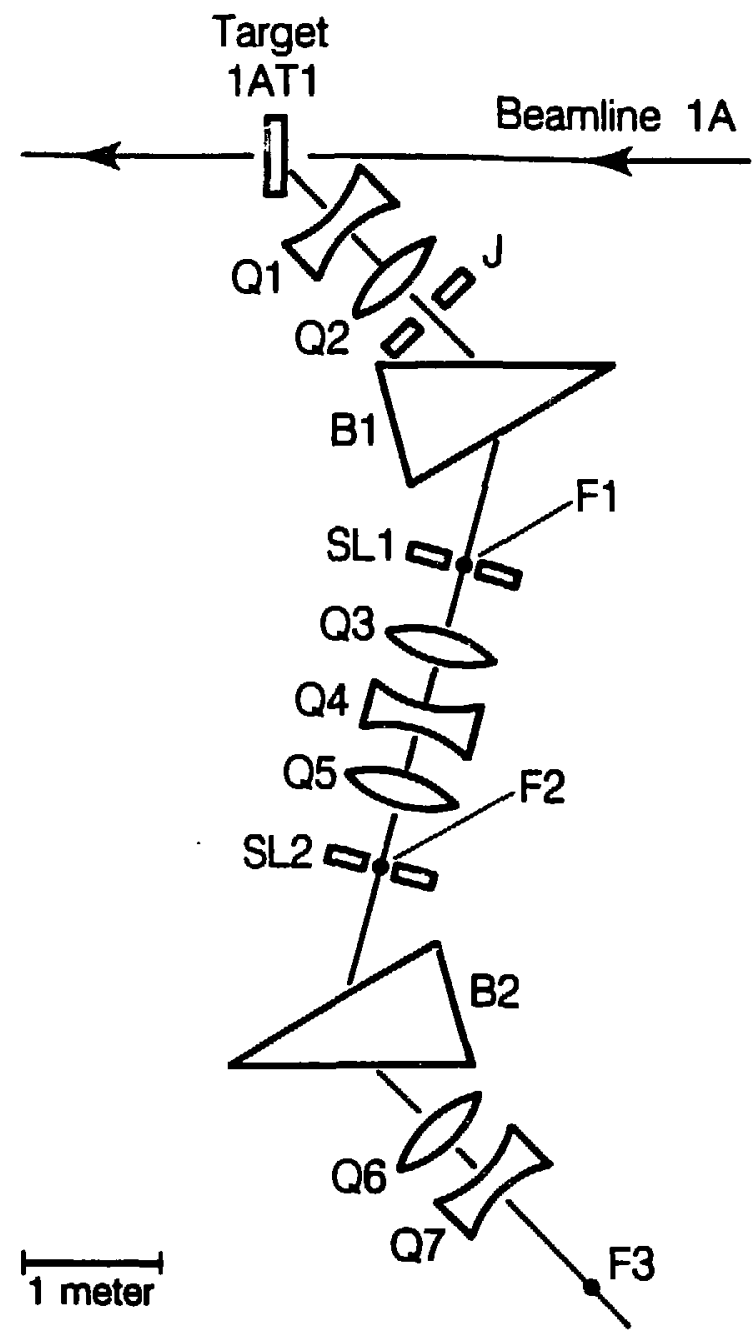

XBL 858-11662

FIG. III.2. The M13 beamline at TRIUMF. $B 1$ and $B 2$ are dipoles; Q1-Q7 are quadrapoles; F1-F3 are foci; the slits SL1 and SL2, and the jaws $\mathrm{J}$ have both horizontal and vertical components. 


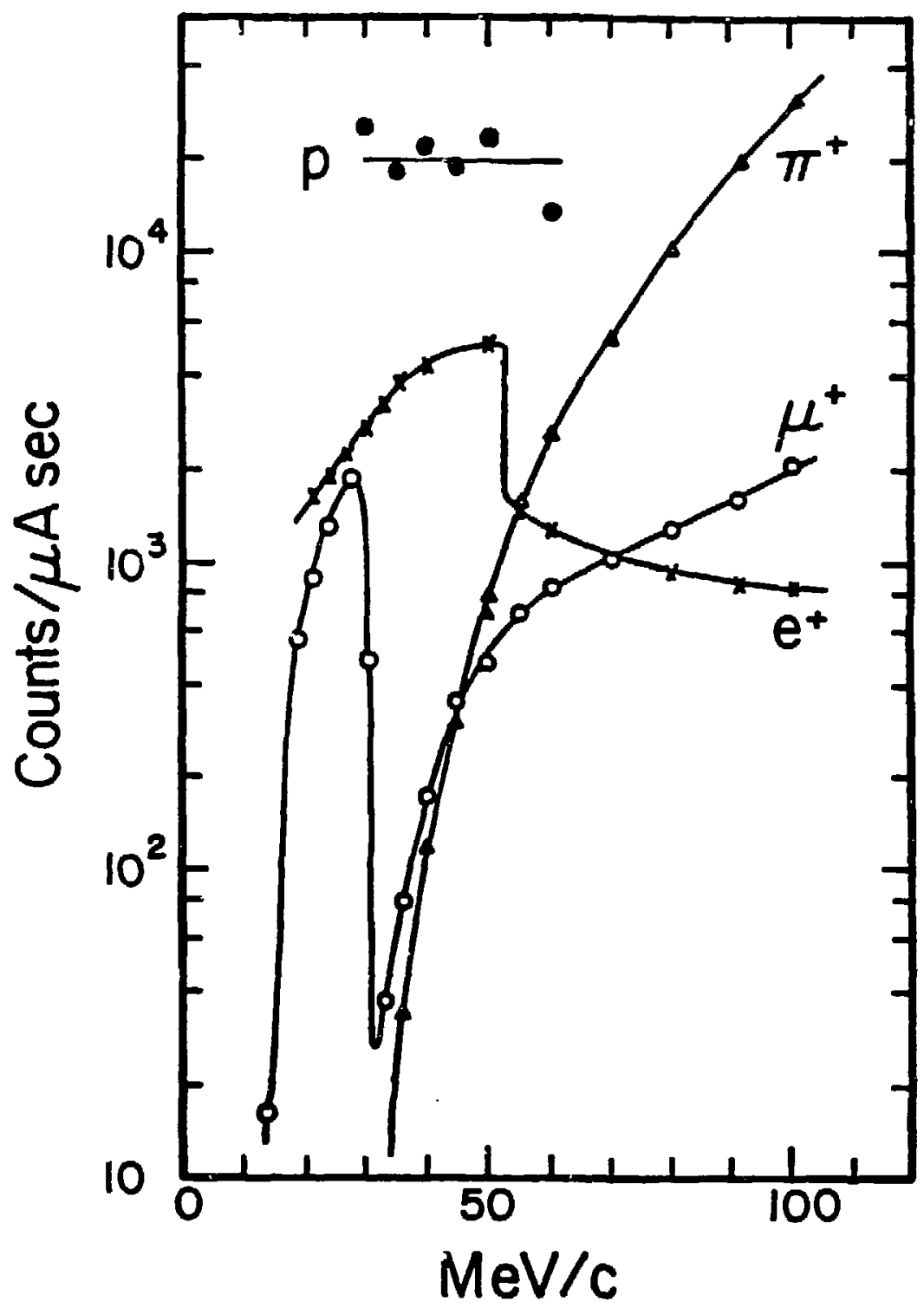

FIG. III.3. Positive particle fluxes versus beamline momentum settings in the M13 beamline at TRIUMF (taken from Ref. 26). The data was collected with all slits and jaws in the beamline fully open. 
constitute over $98 \%$ of the muon flux. In the absence of right-handed currents and scattering they are expected to be fully polarized. The remaining $1.5 \%$ component of the muon flux at this beamline setting sonsjists of "cloud" muons, produced by pions decaying in flight. The cloud muons are not highly polarized, and hence were removed from the event sample.

The cut to eliminate cloud muons was made possible by the pulsed structure of the primary proton beam. At TRIUMF protons bombard the primary target at $43 \mathrm{~ns}$ interval.s. The cloud muons must be produced in close proximity to the production target, i.e. promptly within few ns of the 2-5 ns beam bursts, if they are to be transported by the M13 beamline. Since the production of cloud muons is restricted to a small time interval in the $43 \mathrm{~ns}$ cycle, they could be eliminated from the event sample by a cut on particle arrival time at the stopping target with respect to the cyclotron $\mathrm{RF}$ signal.

The rate of particle arrival at the stopping target with respect to the cyclotron RF signal is shown in Fig. III.4 for the beamline settings of $29.5 \mathrm{MeV} / \mathrm{c}$ and $30.5 \mathrm{MeV} / \mathrm{c}$. For the beamline setting of 29.5 MeV/c (Fig. III.4a), the arrival rate is dominated by the surface muons, which results in an exponential time distribution corresponding to the pion lifetime. For the beamline tuning of 30.5 MeV/c (Fig. III.4b), there are two isolated peaks corresponding to the cloud muons and pions. The cloud muons and pions were eliminated from the event sample by a timing cut, shown by the shaded regions in Fig. III. 4.

Aside from muons and pions, the other particles in the beamline 

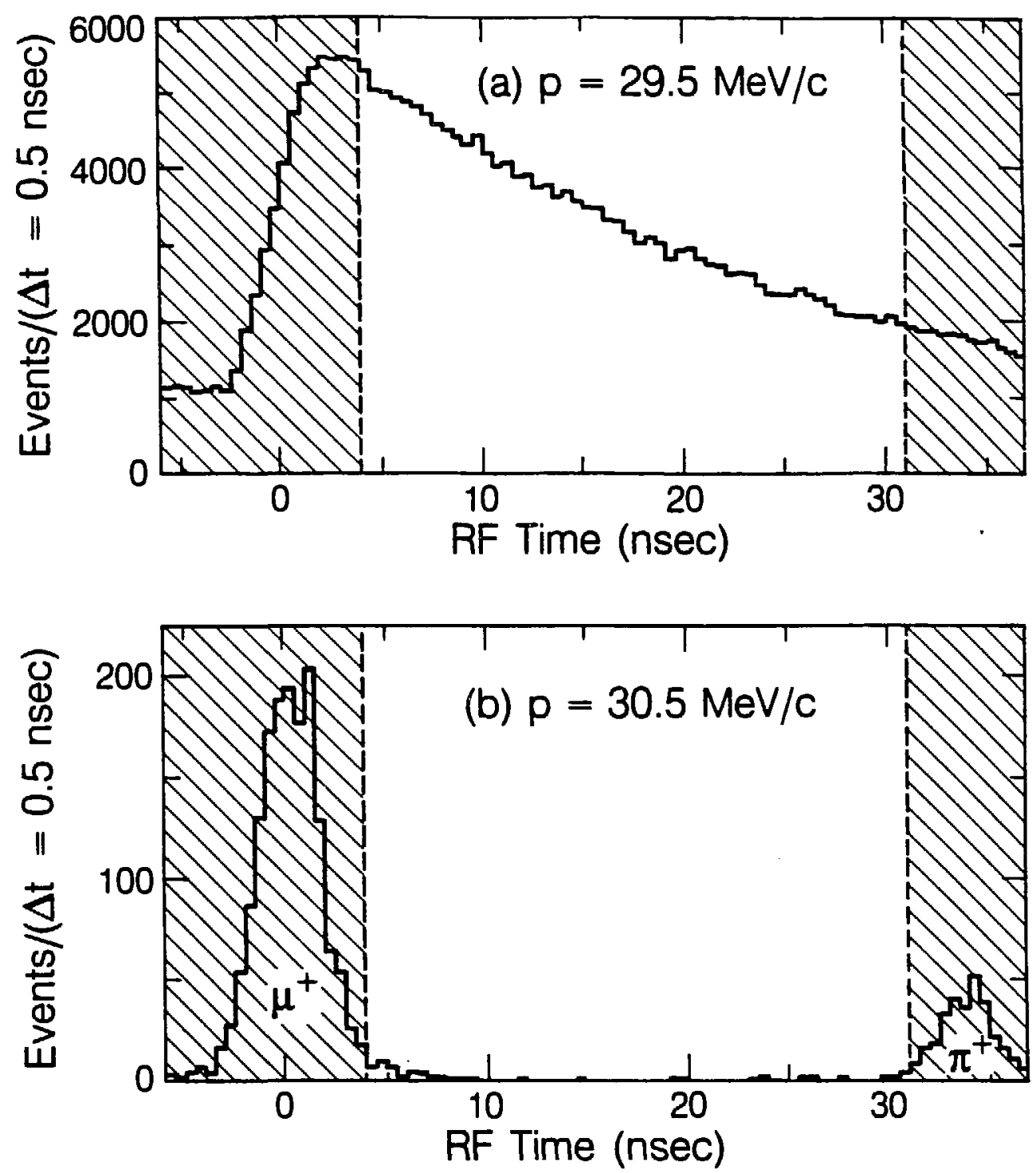

XBL 858-11664

FIG. III.4. Beam particle arrival times at the stopping target with respect to the cyclotron $\mathrm{RF}$ cycle at (a) $29.5 \mathrm{MeV} / \mathrm{c}$ and (b) $30.5 \mathrm{MeV} / \mathrm{c}$. The shaded regions, containing almost all of the cloud $\mu^{+}$and prompt $\pi^{+}$contaminations, were rejected. 
are protons and positrons. Protons stopped in the beaml ine vacuum window and did not reach the detectors. Positrons passed through the stopping targets and therefore were rejected by the trigger requirement.

\section{III.C. Muon Deceleration and Thermalization}

Before describing the muon slowing down process, let us briefly review the suppression of muon depolarization in muonium by the longitudinal magnetic field aligned with the nominal beam direction. The magnetic field direction will define the axis of quantization. The energy eigenstates of muonium will be expressed in terms of a variable $y=|B| /(1585 G)$, and a "natural" basis $\left|m_{\mu} m_{e}\right\rangle$ :

$$
\begin{aligned}
& \mid++> \\
& s|+->+c|^{-+>} \\
& c|+->-s|^{-+>} \\
& \mid-->
\end{aligned}
$$

where the compact notation $\mid+\rightarrow$ means $\left|m_{\mu}=+1 / 2, m_{e}=-1 / 2\right\rangle$, etc., and

$$
c=(1 / \sqrt{2})\left[1+y\left(1+y^{2}\right)^{-\frac{1}{2}}\right]^{\frac{1}{2}} \quad s=(1 / \sqrt{2})\left[1-y\left(1+y^{2}\right)^{-\frac{1}{2}}\right]^{\frac{1}{2}} .
$$

For simplicity, suppose that all muons arrive with a spin parallel to the field. Then half of the muonium ensemble is formed in the state $|++\rangle$ and half in the state $|+-\rangle$. The state $|++\rangle$ is stationary, and hence the muon polarization of half of the ensemble is preserved. The polarization for the other half of the ensemble oscillates between

$$
P_{\mu}=\left(c^{2}-s^{2}\right)^{2}-4 s^{2} c^{2}=\left(y^{2}-1\right)\left(y^{2}+1\right)^{-1}
$$


and $P_{\mu}=1$ with a frequency $\omega=2 \cdot 3\left(1+y^{2}\right)^{\frac{1}{2}} \mathrm{GHz}$. The net polarization is thus $P_{\mu}=(1 / 2)\left(2 y^{2}+1\right)\left(y^{2}+i\right)^{-1}$, and therefore the magnetic field reduces muon depolarization in muonium to $(1 / 2)\left(1+y^{2}\right)^{-1}$.

Muons reached the stojping target with a kinetic energy of $-3.3 \mathrm{MeV}$, after traversing $50 \mathrm{mg} / \mathrm{cm}^{2}$ of material (mostly windows, wires and gas) upstream of the target. The main energy-loss processes, as $\mu^{+}$slows down to thermal velocities, depend on the $\mu^{+}$ kinetic energy. ${ }^{27}$ For kinetic energies $E>2-3 \mathrm{keV}$ the energy loss is due to scattering with electrons, and $\mu^{+}$are partially depolarized through spin exchange with the unpolarized electrons of the medium. ${ }^{28}$ A correction to the result for $\xi P_{\mu} \delta / \rho$ due to such depolarization will be computed in section VI.C. At $E=2-3 \mathrm{keV}$ the $\mu^{+}$ velocity is comparable to that of the valence electrons of the medium, and muons begin to capture and lose electrons rapidly, forming a succession of short-ilved muonium $\left(\mu^{+} e^{-}\right)$states. Again, energy is lost in collisions with electrons. Below $E \approx 200 \mathrm{eV}$ the energy loss is due to collisions of a stable muonium atom with atoms and molecules. The time spent by the decelerating $\mu^{+}$in muonium states $\left(-10^{-12} \mathrm{sec}\right)$ is too short for the hyperfine transitions to cause any appreciable depolarization.

The state in which $\mu^{+}$is finally thermalized depends on the medium. In many non-metals muons are thermalized as muonium. In metals, however, the $\mu^{+}$are thermalized in a quasi-free state, because the high conduction electron concentration effectively screens the $\mu^{+}$from interactions with individual electrons. For this 
reason most of our data was collected with high-purity $(\geq 99.99 \%)$ metal foils $(\mathrm{Ag}, \mathrm{Al}, \mathrm{Au}, \mathrm{Cu})$ as stopping targets (target thicknesses are tabulated in Appendix A). A small amount of data was collected with liquid He, where muonium is disfavored in the final state due to a large difference between the ionization potential of He $(24.6 \mathrm{eV})$ and muonium $(13 . \overline{\mathrm{eV}})$. The energetically favored final state in liquid He (in which the $\mu^{+}$polarization is preserved) is the molecular ion $\mathrm{He}^{+}$with binding energies ${ }^{29}$ of $1.9 \mathrm{eV}$ for the ground state and $1.2 \mathrm{eV}$ for the first vibrational state. (We were therefore surprised to $f$ ind in our experiment that liquid He is $12 \%$ depolarizing without the strong longitudinal field. ${ }^{12}$ A possible explanation is that if muons are thermalized as zuonium, they survive in this form for a considerable time because of the improbability of encountering a $\mathrm{He}^{+}$ ion with which to recombine as $\mathrm{He}^{+}+\mu^{+} \mathrm{e}^{-}+\mathrm{He}^{+}$. )

Although the strong longltudinal field is sufficient to quench $\mu^{+}$ depolarization in muonium, it cannot 'hold' the spins of quasi-free muons in metal targets. The energy difference between states where the muon spin is parallel or anti-parallel to the $1.1-\mathrm{T}\left(0.30^{-T}\right)$ field is only $\Delta E=6.2 \times 10^{-7} \mathrm{eV}\left(1.7 \times 10^{-7} \mathrm{eV}\right)$, while the room temperature thermal energy is $\mathrm{kT}=2.6 \times 10^{-2} \mathrm{eV}$. Relaxation of the muon spins toward the equilibrium situation, where the number of spins parallel or anti-parallel to the applied field are almost equal, requires the presence of oscillating magnetic fields with frequency $\omega=9.4 \times 10^{8} \mathrm{~s}^{-1}$ for $1.1-\mathrm{T}$ longitudinal field $\left(2.6 \times 10^{8} \mathrm{~s}^{-1}\right.$ for $0.30-T$ ). Such fields may be provided by the nuclear magnetic dipole moments. The stopped muon polarization would then decay 
exponentially towards thermal equilibrium with the characteristic spin-lattice relaxation time $T_{1}$. In section $V$ we will look for possible evidence of spin relaxation by comparing the results for $\xi P_{\mu} \delta / p$ from different time intervals.

III.D. The Muon Polarimeter

The muon polarimeter is shown schematically in Fig. III.1. Muons traversed about $50 \mathrm{mg} / \mathrm{cm}^{2}$ of material before being stopped in metal foil or liquid He targets, placed on the axis between the first two coils of the solenoid magnet. The solenold magnet served two purposes. The front portion produced a $0.30-\mathrm{T}$ or $1.1-\mathrm{T}$ longitudinal spin-holding field aligned with the nominal beam direction. The strong longltudinal field quenched muon depolarization in muonium. The back portion, with a bore of 11 - cm radius, served as a $0.5 \mathrm{~T}-\mathrm{m}$ solenoidal field lins, focusing the decay positrons onto the dipole magnet spectroreter. The iron configuration in the stopping target region was designed to produce nearly axial field. The cosine of an angle between the field and the solenoid axis was $>0.99999$ in the fiducial target area.

The experiment was run in two modes, corresponding to two different fleld orientations in the target region. In the spin-holding mode a strong longitudinal field was applied to suppress muon depolarization. The spin-held data were used to measure the decay rate at the endpoint. In the spin-precessing mode, the longitudinal field was nulled to within $\pm 2 \mathrm{G}$, and a $70-G$ or $110-G$ 
vertical field, transverse to the beam direction, was applied. The vertical field was prodused by an additional water-cooled $\mathrm{Cu}$ coil, consisting of four horizontal sections transverse to the beam direction. In the transverse field of 70 (110) $G$ the muon spins precessed about the vertical axis at a frequency of $0.95(1.5) \mathrm{MHz}$. After appropriate cuts on muon decay time, the spin-precessed data is equivalent to the unpolarized, 1.e. $P_{\mu}=0$, data, and hence it is used here for the momentum calibration of the spectrometer.

The residual longitudinal field in the target region was nulled to within \pm 2 G by means of a small reverse current applied to the upstream solenoid coils. The null condition was indicated by the maximal ratio of events to stopped $\mu^{+}$in $R$ un 1 , and by field measurements in Runs 2 and 3.

The primary design goal of the positron spectrometer was to achieve a high momentum resolution, while maintaining an acceptance of $\geq 250 \mathrm{msr}$ in combination with the target solenoid. In order to minimize the adverse effects of multiple Coulomb scattering on momentum resolution, a horizontally focusing spectrometer was chosen, which bent the $x=1 \mathrm{e}^{+}$trajectories by $98^{\circ}$. The $37 \mathrm{in}$. diameter dipole magnet with approximate cylindrical symmetry (originally used by Sagane et al. ${ }^{30}$ ) provided nearly symmetric point-to-point focusing for particles originating in a focal plane at a distance of -1 from the center of the magnet. The volume between the conjugate focal planes was evacuated and the vacuum windows were positioned close to the focal planes.

The combined solenoid lens-spectrometer magnet system accepted 
particles over a $\pm 15 \%$ momentum range ard decay angles relative to the solenoid axis of $\cos \theta>0.95$. In the final analysis we use events only from a sub-portion of this phase space.

The spectrometer magnet was powered continuously, and the NMR-monitored central field of $0.32-T$ drifted by less than one gauss over a period of several weeks. The absolute momentum calibration of the spectrometer was obtained from the position of the $x=1$ edge in the spin-precessed data, and subsequently this calibration was used to fit the spin-held data. To minimize the effect of a drifting spectrometer field and other possible drifts on the $x$ scale calibration, the target field was switched approximately hourly between the spin-holding and spin-precessing orientations. The residual longitudinal field was nulled each time the change was made to the spin-precessing mode.

Particle trajectories in the target region (see inset of Fig. III.1) were measured by the proportional chambers P1 and P2 for the incoming muons, and by the proportional chamber P3 and driftchambers D1 and D2 for the outgoing poșitrons. The scintillation counters S1 (0.005 in. thick), just upstream of P1, and S2 ( $0.010 \mathrm{in}$. thick), just downstream of P3, provided the trigger signals. The thickness of the scintillation counter S1 was kept to a mimimum to minimize the chance of a muon stopping upstream of the target. The veto scintillation counters V1 and V2 (0.125 in. thick), just in front of $\mathrm{S} 1$ and behind $\mathrm{S} 2$ respectively, had 1.5 in. diameter aperture-defining holes around the solenoid axis. All scintillation counters were viewed from left and right by photomultipliers. 
Positron track coordinates outside the spectrometer focal planes were measured by low mass driftchambers D3 and D4. A scintillation counter 53 was placed behind the chamber D4 to provide trigger signals.

The proportional chambers and driftchambers were run on methane-8\% methylal gas mixture, selected to minimize Coulomb scattering. Chamber resolutions and efficlencies will be discussed in section IV on event reconstruction.

\section{III.E. The Trigger}

The trigger logic was based on fast signals from the scintillation counters and the cathode planes of the proportional chambers. A trigger required the signature of a particle stopping in the target, followed, in delayed coincidence of $0.1-10 \mu \mathrm{s}$, by a positron, registered by detectors downstream. With detector signals denoted by their symbols in Fig. III.1, the trigger requirement was $\mathrm{P} 1 \cdot \overline{\mathrm{V} 1} \cdot \mathrm{S} 1 \cdot \mathrm{P} 2 \cdot \overline{\mathrm{P} 3} \cdot \overline{\mathrm{S} 2} \cdot \overline{\mathrm{V} 2}$ in delayed coincidence with $\mathrm{P} 3 \cdot \mathrm{S} 2 \cdot \mathrm{S} 3 \cdot \overline{\mathrm{V} 1} \cdot \overline{\mathrm{V} 2}$. The scintillation counter S1 had a greater amount of material in the muon path than did the proportional chamber $\mathrm{P} 2$, and consequently it was placed upstream of P2 to minimize the probability of a muon stopping in the last detector element required for the muon part of the trigger. The high voltage on the scintillation counter S1 was set to make it efficient for muons, but not for the beam positrons, which deposited 8 times less energy.

Events were tagged if a particle was detected upstream of the 
stopping target within $0.3-10 \mu s$ after a muon scop. Such events had a high probability for the decay positron being produced by an extra muon in the target, rather than the muon causing the trigger. These events were rejected in analysis if the extra particle was detected before the decay positron.

Beam positrons, which constitute $-60 \%$ of the $M 13$ beam flux for the beamline setting of $29.5 \mathrm{MeV} / \mathrm{c}$, passed through the stopping targets and apparatus, and were stopped in lead shielding next to the low momentum side of the vacuum tank in the spectrometer magnet. The beam positrons did not satisfy the particle stop requirement, and only an insignificant fraction of them reached the $\mathrm{s} 3$ counter to mimic decay positrons. The beam momentum of $29.5 \mathrm{MeV} / \mathrm{c}$ was much lower than the minimum positron momentum of $49 \mathrm{MeV} / \mathrm{c}$ for events used in the data analysis. Hence, beam positrons did not constitute a background. 


\section{EVENT RECONSTRUCTION AND SELECTION}

Track reconstruction was relatively simple since there was only one incoming and one outgoing track, and all wire chamber planes were over $90 \%$ efficient with little noise. However, special attention had to be paid to the reconstruction of curved tracks in the solenoid magnet. The curved tracks were fitted using the first-order optics approximation for the cylindrically symmetric fields, as described below.

Initially all $e^{+}$track segments were fitted with straight lines. Positron track segments were found separately in the horizontal and vertical projections in the 3 groups of wire chamber planes (refer to Fig. III.1): P3, D1, D2 (5 vertical and 5 horizontal wire planes), D3 ( 6 and 6 ), and D4 ( 6 and 4 ). All possible hit combinations were considered, and a track with a minimum chi-square was selected among those that had hits in the maximum number of planes. A combination of hits was considered to be a track if the total $x^{2}$ was below a maximum value corresponding to a hit accuracy of $-1 \mathrm{~mm}$ in D3 and D4 and $-2 \mathrm{~mm}$ in P3 through D2.

In $99 \%$ of the triggers tracks were found in all six segments. 13\% of the triggers were rejected to avold ambiguities due to high or low hit multiplicities associated with the $\mu^{+}$or $e^{+}$track. To achieve greater accuracy in the $\cos \theta$ measurement positron tracks were required to have unambiguous hits in both the horizontal and vertical planes of $\mathrm{P3}$, and at least 3 hits were required in each projection of the $\mathrm{e}^{+}$track inside the solenoid. $95 \%$ of events in the final sample 
had the $e^{+}$track portion inside the solenoid recorded by at least 9 wire planes.

Muon tracks were accepted if they were unambiguous, or if there were no more than two hits in one and only one of the P2 planes. In the latter case ( $5 \%$ of the final sample) we selected the track which in extrapolation to the stopping target was closest to the decay vertex indicated by the $e^{+}$track extrapolation.

Track segments in the solenoid were then refitted in both projections simultaneously in the first-order optics approximation for cylindrically symmetric fields.31 In this approximation particle trajectories through the solenoid are determined from initial conditions by means of transfer matrices, computed from magnetic rigidity and field values on the solenoid axis. Curved tracks could therefore be fitted without detailed step-by-step computation of orbits in the magnetic field. Incoming and outgoing tracks were extrapolated to the stopping target, so that the track angles could be determined at the decay vertex. It was found in the Monte-Carlo simulation based on propagating the particles through the magnetic field in small steps that, in the absence of scattering and chamber resolution effects, $\cos \theta$ was determined extremely accurately using the first-order optics approximation (see section VII.D).

The non-linear time-distance relationships in the driftchambers were obtained separately for each plane of every chamber. All chamber planes were aligned in the transverse direction; the residual means were less than $50_{\mu}$. In the initial approximation the time-distance relationships were found by integrating the flux ard 
assuming uniform cell illumination. They were then dynamically fine-tuned by making small adjustments based on track residuals. The procedure converged after 1500 events. Curved tracks were fitted in the first-order optics approximation. Residuals of $0<500 \mu$ were achieved in D1 and D2, with the exception of two planes in D1 where o was $=700 \mu$. Residuals in D3 and D4 had $\sigma<250 \mu$.

All proportional chambers had $2 \mathrm{~mm}$ wire spacings, with up to 3 consecutive wires considered as a single acceptable hit. Events with 4 or more consecutive wires hit were rejected.

Positron tracks were required to link in the following extrapolations: inside the solenoid magnet, both in radial and azimuthal positions; in vertical slope and vertical position inside the spectrometer magnet; and in impact parameter with respect to the central axis of the approximately cylindrically symmetric field of the spectrometer. Muon and positron extrapolations to the decay vertex at the stopping target were required to match within $4 \mathrm{~mm}$ radius. Track linkage requirements resulted in a $30 \%$ loss of events. Events near the edges of geometrical acceptance were cut to avoid particles that were scattered back into the apparatus (20\% event loss). Other small fiducial cuts were made to insure event unambiguity.

The horizontal focusing property of the spectrometer allowed the $\mathrm{e}^{+}$momentum to be reconstructed from the sum of horizontal coordinates at the two conjugate focal planes. This sum was empirically corrected with momentum-dependent first or second-order terms in three variables: mean vertical deviation from the median 
plane of the spectrometer, impact parameter with respect to spectrometer axis, and vertical position at the spectrometer exit. Inttially, the corrections were obtained from special calibration runs, when beamline momentum bite was reduced to $0.5 \% \Delta p / p$, and straight-through beams of positrons, in 3 to $5 \%$ steps in momentum, passed through the apparatus. The corrections were then $f$ ine-tuned by requiring the edge position in unpolarized, i.e. spin-precessed, data to be identical for every portion of spectrometer phase-space, partitioned in the above mentioned three variables. For calibration purposes, the spin-precessed data were collected at several values of spectrometer current, varying by -15 to $+5 \%$ from the optimum in 3 to 5\% steps. Momentum resolution achieved was approximately Gaussian, with $0=0.13 \%$.

Monentum dispersion of $1.056 \pm 0.008 \%$ was obtained from the straight-through calibration runs taken with spectrometer currents varying from 42 to $100 \%$ of the optimal current in $f$ ive 15 to $20 \%$ steps, and also from the edge positions found in runs at different settings of spectrometer current. The uncertainty in dispersion is based on agreement between five calibration curves, obtained at different times during the three data-taking runs. 


\section{v. DATA ANALYSIS}

The analysis exploited the fact that near the endpoint the muon decay spectrum can be expressed as a linear combination of two pure V-A spectra, one with $P_{\mu} \cos \theta=0$, and another with $P_{\mu}=\cos \theta=1$. This is discussed in section II. The relevant equations are (2.2), (2.5) and (2.6). The result for $\xi \mathrm{P}_{\mu} \delta / \rho$ was extracted from the rate at the spectrum endpoint $r(\theta)$, given by equation (2.5).

Fitting was performed with a double-precision version of the MINUIT program, and was based on minimizing the maximum-likelihood Poisson statistics $\chi^{2}$, defined by

$$
x^{2}=2\left[\left[e_{i}-o_{i}+o_{i} \ln \left(o_{i} / e_{i}\right)\right]\right.
$$

where $o_{i}$ and $e_{i}$ are the observed and expected number of events respectively for the $i^{\text {th }}$ bin.

The data were fitted separately in $f i v e \cos \theta_{e}$ bins as explained below. For each $\cos \theta_{\mathrm{e}}$ bin the fitting proceeded in two stages. First, the spin-precessed data were fitted to calibrate the spectrometer and determine its resolution and acceptance functions. The position of the $x=1$ edge in the spin-precessed data determined the location of the $x=1$ point on the reconstructed momentum scale; the edge sharpness determined the spectrometer momentum resolution. In the second stage, the spin-held data were fitted using the parameters found in the fit of the spin-precessed data. We now consider the fitting procedure in detail. 


\section{V.A. The Fitting Procedure}

Here we describe the fitting performed for each of the five $\cos \theta_{e}$ bins.

For the spin-precessed data, cuts on muon decay time were adjusted to obtain an equivalent of the unpolarized, i.e. $P_{\mu}=0$, data sample. The resulting unpolarized spectrum, binned in $x$ bins 0.001 wide (see Fig. V.1), was fitted from $x=0.93$ to $x=1.01$ as

$$
N_{1} S_{V-A}(x, 0) A(x)
$$

where $N_{1}$ is a free normalization, and $S_{V-A}(x, 0)$ is the pure $V-A$ decay spectrum with $P_{\mu} \cos \theta=0$. $A(x)$ is a quadratic acceptance function given by

$$
A(x)=1+B_{1}(1-x)+B_{2}(1-x)^{2}
$$

where the linear and quadratic coefficients $B_{1}$ and $B_{2}$ are also free parameters.

If $\bar{\rho}[i . e .1-(4 / 3) \rho]$ is non-zero, the unpolarized spectrum does not match the $V-A$ unpolarized spectrum $S_{V-A}(x, 0)$, and the acceptance function (5.2) will absorb the difference in spectral shapes. As a result, the linear coefficient $B_{1}$ in equation (5.2) gets a systematic shift of $+6 \tilde{\rho}$, and a quadratic coefficient $B_{2}$ a shift of $\left(6 B_{1}-12\right) \tilde{\rho}$. The world-average value of $\vec{p}$ is $-.0023 \pm .0035$. Fortunately, an error in $B_{1}\left(B_{2}\right)$ propagates into an error in $\xi P_{\mu} \delta / \rho$ with a factor of $10^{-3}$ $\left(1.5 \cdot 10^{-5}\right)$, and hence this effect can be ignored.

The fitting function (5.1) was smeared to reflect spectrometer resolution and positron energy-loss straggling in the target (the amount of target material in positron path varied from 23 to 


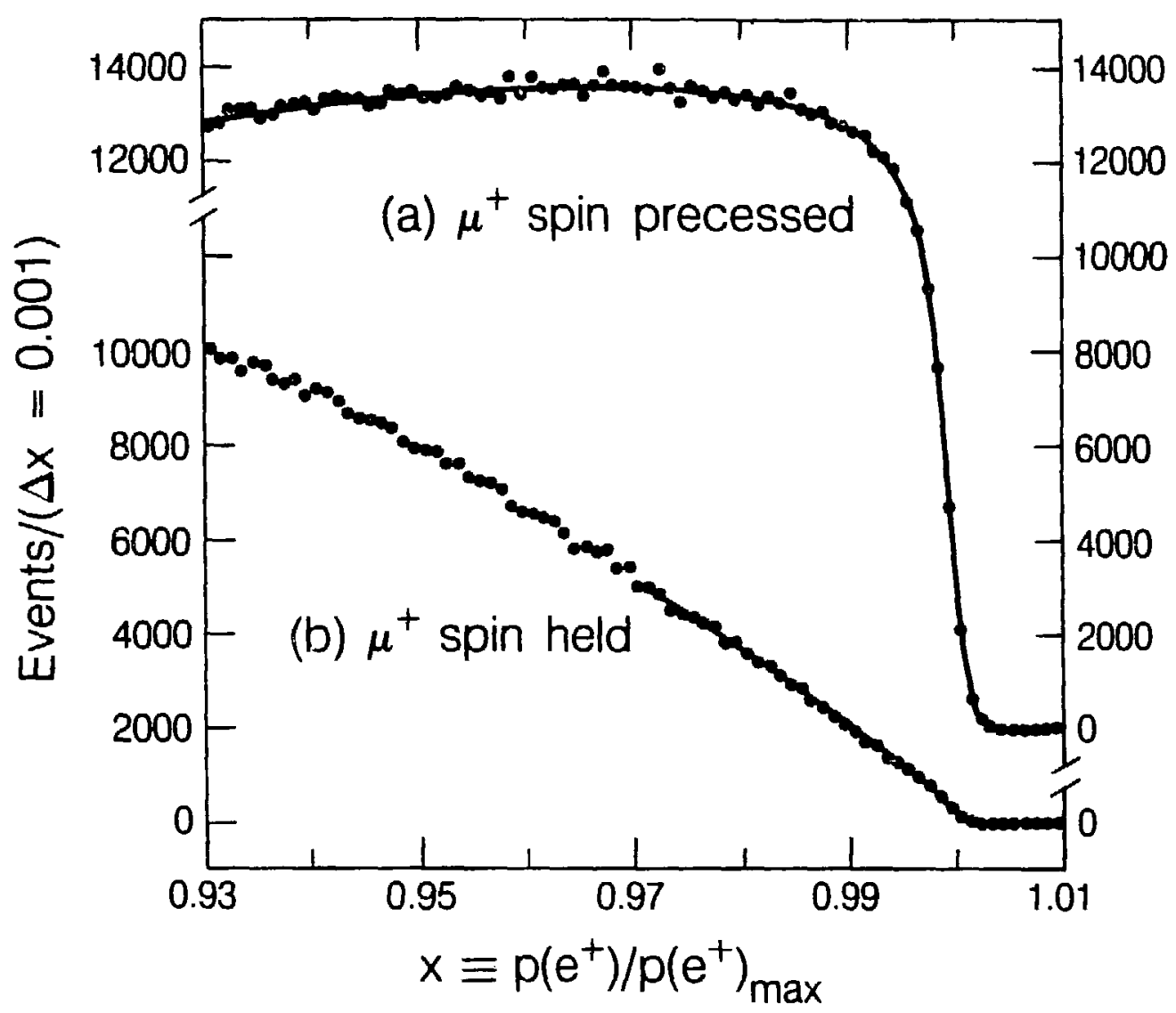

XBL 8512.11759

FIG. V.1. Measured positron spectra for (a) spin-precessing mode and (b) spin-holding mode. $x$ is the reduced positron momentum. The solid curves show the fitted $x$ ranges $(x>.93$ for the spin-precessed and $x>.97$ for the spin-held data). The edge in (a) corresponds to a resolution with gaussian part of <.15\% rms. The spectra shown contain the data from all targets. Fits were performed for each target separately in $5 \cos \theta_{e}$ bins, as described in the text. 
$170 \mathrm{mg} / \mathrm{cm}^{2}$; see Appendix A) and in $=200 \mathrm{mg} / \mathrm{cm}^{2}$ of material downstream up to the spectrometer vacuum tank window.

The spectrometer (i.e. $x$ ) resolution was parametrized by a normalized sum of three gaussians with standard deviations $a, \sqrt{3} 0$ and 30. The $\sqrt{30}$ and 30 gaussians were introduced in order to obtain a better $f$ it to the tails of the resolution in $x$, and they usually had small coefficients in the normalized sum. The resolution o was typically $0.13 \%$. The result for $\xi P_{\mu} \delta / \rho$ was relatively insensitive to the number of free parameters in the resolution function, changing by less than $10^{-4}$ when a single gaussian was used rather than all three.

There were a total of 7 free parameters in the fit of the spin-precessed data: the normalization $\mathrm{N}_{1}$, the linear and quadratic coefficients $B_{1}$ and $B_{2}$ in the acceptance function, the 3 resolution parameters, and the position of the $x=1$ point on the reconstructed momentum scale.

Despite the large momentum acceptance of the apparatus, the spin-precessed data were fitted only from $x=0.93$ and the spin-held data only from $x=0.97$. A short extension of the $x$ range below $x=0.93$ for the spin-held data had nc effect on the result for $\xi P_{\mu} \delta / \rho$. A significant extension would have required more free parameters to fit acceptance, and would not have led to a decrease in the statistical error on $\xi_{P_{\mu}} \delta / p$. The situation was similar for the spin-held data. Here the extension of the $x$ range below 0.97 would have required an introduction of a free parameter to represent a possible difference in acceptance between the spin-precessed and spin-held data sets. Since the acceptance parameters correlated with $r(\theta)$, the rate at the 
endpoint, the statistical error on $\xi P_{\mu} \delta / \rho$ would have increased. Furthermore, the systematic error due to possible differences in $x$ acceptance between the two data sets increased quadratically with the $x$ range for the fit of spin-held data. The range $x \geq 0.97$ for the spin-held data was optimal for the smallest combined (1.e. statistical and systematic) error.

The spin-held data, also shown in Fig. V.1, were fitted using the values for the $x$ acceptance, $x$ resolution and $x=1$ calibration point found for the spin-precessed data. In the second stage of fitting the two data sets were fitted simultaneously to allow the uncertainties in the $x=1$ calibration and $x$ acceptance to contribute ( $\sim 3 \%$ of the error) to the statistical error on $\xi P_{\mu} \delta / \rho$. Since the resolution in $x$ was determined primarily by the sharpness of the $x=1$ edge in the spin-precessed data, the resolution parameters in the combined fit were fixed to the values found when the spin-precessed data were fitted alone. Fixing the resolution parameters shortened the overall computer time required by a factor of 3 . It was checked that fixing the resolution parameters in the combined fit did not affect either the result or the errors on $\xi_{2} P_{\mu} \delta / \rho$.

In the combined fit the spin-precessed data were fitted to the same function (5.1) as before, except that the resolution parameters were fixed. The spin-held data, also binned in $\mathrm{x}$ bins 0.001 wide (see Fig. V.1), was fitted according to equation (2.2), from $x=0.97$ to $x=1.01$, as

$$
N_{2}\left[(1-r(\theta)) S_{V-A}(x, 1)+r(\theta) S_{V-A}(x, 0)\right] A(x)
$$

where $\mathrm{N}_{2}$ is a free normalization, $\mathrm{S}_{V-A}(x, 1)$ and $\mathrm{S}_{V-A}(x, 0)$ are the 
pure V-A spectra at $P_{\mu} \cos \theta=1$ and $P_{\mu} \cos \theta=0$ respectively, $A(x)$ is the quadratic acceptance function (5.2), and the free parameter $r(\theta)$ is the relative rate at the endpoint. The function (5.3) was smeared in the same manner as for the spin-precessed data to reflect the spectrometer resolution and positron energy-loss straggling.

There were a total of 6 free parameters in the combined fit. They were the $x=1$ calibration point, $\mathrm{N}_{1}, \mathrm{~B}_{1}, \mathrm{~B}_{2}$, all determines primarily by the spin-precessed data, and $\mathrm{N}_{2}, r(\theta)$, determined by the spin-held data.

The result for $\xi P_{\mu} \delta / \rho$ was extracted from $r(\theta)$, which, according to the equation $(2.5)$, is $J$ ust $1-\left(\xi P_{\mu} \delta / \rho\right) \cos \theta$. Since the data were fitted in subsets in which the variation in $\cos \theta$ was much greater than the error on $r(\theta)$, and since any error in $\cos \theta$ propagates into the same error in $\xi \mathrm{P}_{\mu} \delta / \rho$, we must consider the question of what value should be assigned to $\cos \theta$ under such circumstances. We rewrite $r(\theta)$ as

$$
r\left(\theta_{\mathrm{av}}\right)=1-\xi \mathrm{P}_{\mu}{ }_{\mathrm{p}}^{\delta} \cos \theta_{\mathrm{av}} \text {. }
$$

The complication is that $\cos \theta_{a v}$ is not just $\cos \theta$ averaged over the data, as will be explained in section V.B.

Let us mention here the main point of that section: $\cos \theta_{a v}$ was computed from $\cos \theta_{\mu} \cos \theta_{e}$ averaged over the spin-held data. Here $\theta_{\mu}$ and $\theta_{\mathrm{e}}$ are the polar angles with respect to the solenold axis for muons and positrons respectively. Since muon spins precessed about the solenoid axis very rapidly (40 Mhz for 0.3-T longitudinal field and $150 \mathrm{Mhz}$ for $1.1-\mathrm{T}$ ) compared to the muon lifetime $\tau_{\mu}=2.2 \mathrm{~ns}$, one 
may think of $\cos \theta_{\mu} \cos \theta_{e}$ as $\cos \theta$ averaged over one spin-precession period $\left(T<<\tau_{\mu}\right):$

$$
\cos \theta_{\mu} \cos \theta_{e}=-(1 / T) \int_{0}^{T} \hat{s}_{\mu}(t) \cdot \hat{p}_{e} d t
$$

where $\hat{s}_{\mu}$ and $\hat{p}_{e}$ are the unit vectors corresponding to the muon spin and positron momentum respectively. Two small corrections had to be added to the data-averaged $\cos \theta_{\mu} \cos \theta_{e}$. They will be discussed in section VI. The reader who does not wish to follow a rather involved discussion in section V.B may now proceed directly to section V.C summarizing the analysis method.

\section{V.B. Computation of $\cos \theta_{\mathrm{av}}$}

Recall that in the expression for the muon decay spectrum $\cos \theta$ was defined as $-\hat{s}_{\mu} \cdot \hat{p}_{e}$, where $\hat{s}_{\mu}$ and $\hat{p}_{e}$ are the unit vectors corresponding to the directions of muon spin and positron momentum at the $\mu^{+}$decay vertex. At the $\pi^{+}$decay vertex the muon spin is parallel or anti-parallel to the muon momentum (massless neutrinos have been assumed; $\mu^{+}$helicity is always -1 for $V-A$ ). For the the moment let us ignore the effects of scattering, finite chamber resolution and finite accuracy of track extrapolation. The corrections to $\cos \theta_{a v}$ due to these factors will be considered in section VI. Therefore, we can assume that the spin direction of muon when it enters a stopping target is given by the extrapolation of the measured muon track, and similarly that the direction of the positron momentum at the decay vertex is given by the extrapolation of the 
measured positron track.

Let $\theta_{\mu}, \psi_{\mu}$ and $\theta_{e}, \psi_{e}$ be the polar and azimuthal angles with respect to the longitudinal field axis for $-\hat{s}_{\mu}$ (the minus sign is put in for convenience) and $\hat{p}_{e}$ respectively. Since muon spins precessed very rapidly in the strong longitudinal field, $\psi_{\mu}$ would have been difficult to measure. However, it was not necessary to measure $\psi_{\mu}$, for it will be shown that it is sufficient to know just $\theta_{\mu}$.

To distinguish the spin direction $\hat{s}_{\mu}$ at the $\mu^{+}$decay vertex from the spin direction when a muon enters the stopping target, let us denote the former by $\hat{s}_{\mu, v}$ and the latter by $\hat{s}_{\mu \text {, ent - We will Ignore }}$ muon scattering in the stopping target, so that $\theta_{\mu, v}$ is identical to $\theta_{\mu, \text { ent }}$, and both will be denoted by $\theta_{\mu}$.

Our goal is to compute the positron spectrum for the spin-held data in terms of the theoretical decay rate, the angular and $\mathbf{x}$ acceptance functions, and the angular distribution of incoming muons. We will now show that for muons reaching a stopping target, the angular distribution of muon spins at the decay vertex, i.e. the $\hat{s}_{\mu, v}$ distribution, is independent of the azimuthal angle $\psi_{\mu, v}$.

Let $Q\left(\theta_{\mu}, \psi_{\mu}\right.$, ent $)$ describe the angular distribution of $\hat{s}_{\mu \text {, ent }}$ for muons reaching a stopping target, after all software cuts relevant to muons have been applied. Muon spins precess about the longitudinal field axis very rapidly (40 Mhz for $0.3-\mathrm{T}$ and $150 \mathrm{Mhz}$ for $1.1-\mathrm{T}$ ) compared to the muon lifetime $\tau_{\mu}=2.2 \mu \mathrm{s}$. This implies that for a muon, arriving with the spin direction given by $\left(\theta_{\mu}, \psi_{\mu}\right.$, ent $)$, the probability of muon spin direction at the decay vertex being $\left(\theta_{\mu}, \psi_{\mu, v}\right)$ is independent of $\psi_{\mu, v}$. Since the $\hat{s}_{\mu, v}$ angular 
distribution, for the muons reaching a stopping target, is independent of $\psi_{\mu, v}$, it can be written as

$$
R\left(\theta_{\mu}\right)=(1 / 2 \pi) \int_{0}^{2 \pi} Q\left(\theta_{\mu}, \psi_{\mu, \text { ent }}\right) d \psi_{\mu, \text { ent }} .
$$

It is important to distinguish the $\hat{s}_{\mu, v}$ distribution for the muons reaching a stopping target from the $\hat{s}_{\mu, v}$ distribution for events in the spin-held data. Although the former distribution is independent of $\psi_{\mu, v}$, the latter distribution does in fact depend on $\psi_{\mu, v}$ if the angular acceptance for positrons is $\psi_{e}$ dependent. It is the former distribution, represented by $R\left(\theta_{\mu}\right)$, which will be needed for the computations below.

The muon decay spectrum may be written as

$$
S(x, \cos \theta)=a(x)+b(x) \cos \theta \text {. }
$$

For the spin-held data in one of the $\cos \theta_{e}$ bins, let $A\left(\theta_{e}, \psi_{e}, x\right)$ denote the total acceptance function for positrons, including the selection process bias (1.e. $\cos \theta_{e}$ must be in a certain range), the acceptance of the apparatus and software cuts. Ignoring statistical fluctuations, the positron spectrum $W(x)$ for the fitted subset of the spin-held data is given by

$$
W(x)-\int[a(x)+b(x) \cos \theta] A\left(\theta_{e}, \psi_{e}, x\right) R\left(\theta_{\mu}\right) d \omega_{e} d \omega_{\mu}
$$

where $d w$ denotes the integration over the angular phase space, i.e. $d \omega_{e}=d\left(\cos \theta_{e}\right) d \psi_{e}$, and $\cos \theta=\cos \theta_{\mu} \cos \theta_{e}+\sin \theta_{\mu} \sin \theta_{e} \cos \psi_{\mu} \cos \psi_{e}+\sin \theta_{\mu} \sin \theta_{e} \sin \psi_{\mu} \sin \psi_{e}$. From now on $\psi_{\mu}$ always denotes $\psi_{\mu}, v$.

Let us make an assumption that $\mathbf{x}$ acceptance is independent of $\cos \theta_{e}$. In practice this was not true, and we will return to this 
issue shortly. Under this assumption, $\mathrm{A}\left(\theta_{\mathrm{e}}, \psi_{\mathrm{e}}, \mathrm{x}\right)$ can be written as a product of two acceptance functions

$$
A\left(\theta_{e}, \psi_{e}, x\right)=A\left(\theta_{e}, \psi_{e}\right) A(x) .
$$

If the spectrum $W(x)$ is now fitted according to $(5.3), \cos \theta$ av is defined by

$$
\begin{aligned}
& N\left[a(x)+b(x) \cos \theta_{a v}\right] A(x)= \\
& \quad \int[a(x)+b(x) \cos \theta] A\left(\theta_{e}, \psi_{e}\right) A(x) R\left(\theta_{\mu}\right) d \omega_{e} d \omega_{\mu}
\end{aligned}
$$

where $\mathrm{N}$ is the normalization. Hence

$$
\cos \theta_{\mathrm{av}}=\frac{\int A\left(\theta_{e}, \psi_{e}\right) R\left(\theta_{\mu}\right) \cos \theta d \omega_{e} d \omega_{\mu}}{\int A\left(\theta_{e}, \psi_{e}\right) R\left(\theta_{\mu}\right) d \omega_{e} d \omega_{\mu}} .
$$

Note that $\cos \theta_{a v}$ is not $\cos \theta$ averaged over the data, which is given by

$$
\langle\cos \theta\rangle=\frac{\int \cos \theta[a(x)+b(x) \cos \theta] A\left(\theta_{e}, \psi_{e}\right) A(x) R\left(\theta_{\mu}\right) d \omega_{e} d \omega_{\mu} d x}{\int[a(x)+b(x) \cos \theta] A\left(\theta_{e}, \psi_{e}\right) A(x) R\left(\theta_{\mu}\right) d \omega_{e} d \omega_{\mu} d x} .
$$

Since $R\left(\theta_{\mu}\right)$ is independent of $\psi_{\mu}$, the $\psi_{\mu}$-dependent terms in $\cos \theta$ vanish in (5.8) after integration over $\psi_{\mu}$, and hence in (5.8) $\cos \theta$ can be replaced by $\cos \theta_{\mu} \cos \theta_{e}$

$$
\cos \theta_{a v}=\frac{\int \cos \theta_{\mu} \cos \theta_{e} A\left(\theta_{e}, \psi_{e}\right) R\left(\theta_{\mu}\right) d \omega_{e} d \omega_{\mu}}{\int A\left(\theta_{e}, \psi_{e}\right) R\left(\theta_{\mu}\right) d \omega_{e} d \omega_{\mu}} .
$$

As mentioned above, one may think of $\cos \theta_{\mu} \cos \theta_{e}$ as $\cos \theta$ averaged over one spin precession period $\left(T<<\tau_{\mu}\right)$ :

$$
\cos \theta_{\mu} \cos \theta_{e}=-(1 / T) \int_{0}^{T} \hat{s}_{\mu}(t) \cdot \hat{p}_{e} d t
$$

Since $A\left(\theta_{e}, \psi_{e}\right)$ was not known a priori, in the analysis $\cos \theta_{a v}$ was computed from $\cos \theta_{\mu} \cos \theta_{e}$ averaged over the spin-held data 


$$
\begin{aligned}
& <\cos \theta_{\mu} \cos \theta_{e}>= \\
& =\frac{\int \cos \theta_{\mu} \cos \theta_{e}[a(x)+b(x) \cos \theta] A\left(\theta_{e}, \psi_{e}\right) A(x) R\left(\theta_{\mu}\right) d \omega_{e} d \omega_{\mu} d x}{\int[a(x)+b(x) \cos \theta] A\left(\theta_{e}, \psi_{e}\right) A(x) R\left(\theta_{\mu}\right) d \omega_{e} d \omega_{\mu} d x} .
\end{aligned}
$$

Two corrections had to be added to $\left\langle\cos \theta_{\mu} \cos \theta_{e}\right\rangle$. One correction is due to the difference between $\left\langle\cos \theta_{\mu} \cos \theta_{e}\right\rangle \operatorname{computed}$ from the measured tracks, and $\left\langle\cos \theta_{\mu} \cos \theta_{e}\right\rangle$ if it were computed from the true values of $\theta_{\mu}$ and $\theta_{e}$. This difference is due to scattering and finite chamber resolution. Since positron scattering depended on the target, this correction was target-dependent. The second correction compensated for the difference between $\left\langle\cos \theta_{e} \cos \theta_{\mu}\right\rangle$, given by (5.11), and the acceptance-weighted average $\cos \theta_{e} \cos \theta_{\mu}$, defined by (5.10). The second correction was target-independent. The computation of these corrections is described in section VI.

Let us now return to the issue of $\cos \theta_{e}$-dependent $x$ acceptance. If $x$ acceptance depends on $\cos \theta_{e}, \cos \theta_{a v}$ is not rigorously defined, since the equality (5.7) can no longer be written. However, if the spectrum $W(x)$ is fitted as in (5.3), with $\cos \theta_{a v}$ computed from $\left\langle\cos \theta_{\mu} \cos \theta_{e}\right\rangle$ and the corrections mentioned above, then the systematic error in $r\left(\theta_{a v}\right)$ will be negligibly small provided that the variation In $x$ acceptance is small for the range of $\cos \theta_{e}$ in the data.

Therefore, in order to minimize the systematic error in $r\left(\theta_{a v}\right)$ due to $\cos \theta_{e}$-dependent $x$ acceptance, the data were fitted in $f$ ive $\cos \theta_{e}$ bins 0.005 wide, $0.9755 \cos \theta_{e} \leq 1$. If the five $\cos \theta_{e}$ bins were fitted together, the systematic error in $r\left(\theta_{a v}\right)$ due to $\cos \theta_{e}$-dependent acceptance would have been $\pm 1 \cdot 10^{-4}$. When each $\cos \theta_{e}$ bin was fitted separately, the systematic error in $r\left(\theta_{a v}\right)$ was smaller than $\pm 0.2 \cdot 10^{-4}$ 
for each $\cos \theta_{e}$ bin. Events with $\cos \theta_{e}<0.975$ were dropped from analysis since they had low statistical power.

The result for $\xi P_{\mu} \delta / \rho$ was obtained when the five values of $r\left(\theta_{a v}\right)=1-\left(\xi P_{\mu} \delta / \rho\right) \cos \theta_{a v}$, taken with asymmetric errors, were used in a fixed slope extrapolation to $\cos \theta_{a v}=1$. It was checked that the result from fitting the $f$ ive $\cos \theta_{e}$ bins separately was consistent with the result obtained when all $\cos \theta_{e}$ bins were fitted together.

\section{V.C. Summary of the Analysis Method}

The data were fitted separately in $f$ ive $\cos \theta_{e}$ bins 0.005 wide, $0.975 \leq \cos \theta_{e} \leq 1$. For each $\cos \theta_{e}$ bin the fitting was performed in two stages. First, the spin-precessed data were fitted from $x=0.93$ according to (5.1). Then the resolution parameters were fixed, and the spin-held data and the spin-precessed data were fitted simultaneously, from $x=0.93$ and $x=0.97$ respect 1 vely, to determine the relative rate at the endpoint for the spin-neld data, given by $r\left(\theta_{\mathrm{av}}\right)$. The spin-held data were fitted according to (5.3) and the spin-precessed data according to (5.1). For each $\cos \theta_{e}$ bin, $\cos \theta_{a v}$ was computed from $\cos \theta_{\mu} \cos \theta_{e}$ averaged over the spin-held data, to which there were added two small corrections. Finally, the five values of $r\left(\theta_{a v}\right)=1-\left(\xi P_{\mu} \delta / \rho\right) \cos \theta_{a v}$ were used in a fixed slope extrapolation to $\cos \theta_{a v}=1$, which yielded $\xi P_{\mu} \delta / \rho$.

The analysis method was checked in two ways.

First, the positron spectra were evaluated numerically in the five $\cos \theta_{e}$ bins for various values of the muon decay parameters $\rho, \delta$, 
$\xi$ and various acceptance functions $A\left(\theta_{e}, \psi_{e}\right)$ and $A(x)$. The incoming muon distribution was taken to be the measured distribution which will be discussed in section VI.B. The spectra were processed through the analysis program and the results for $r\left(\theta_{a v}\right)$ were compared with the prediction $\left[1-\left(\xi P_{\mu} \delta / p\right) \cos \theta_{a v}\right]$, where $\cos \theta_{a v}$ is defined by (5.10). It was verified that for $\tilde{\rho}=\tilde{\delta}=0 \quad[\tilde{\rho}=1-(4 / 3) \rho, \tilde{\delta}=1-(4 / 3) \delta]$ there was always an exact agreement between $r\left(\theta_{a v}\right)$ and the prediction [statistical fluctuations were not introduced in the spectra]. For non-zero $\tilde{\rho}$ and $\tilde{\delta}$ the agreement was to first order in $\tilde{\rho}$ and $\tilde{\delta}$, as expected (see section II).

Second, the same analysis was applied to the data generated in the full Monte-Carlo simulation of the polarimeter. Events were generated assuming $\xi P_{\mu} \delta / \rho=1$. Particles were moved in small steps from the vacuum window to the stopping target for $\mu^{+}$, and from the stopping target to the last wire plane in the driftchamber $D 4$ for $\mathrm{e}^{+}$. Scattering and energy loss were applied at each step. The data from the simulation were processed through the same event reconstruction and selection algorithm as the experimental data. After the two corrections (total of -0.00023 ) to $\cos \theta_{a v}$ the result for $\xi P_{\mu} \delta / \rho$ was $0.99992 \pm 0.00038$, in good agreement with the expected value of 1 . 


\section{V.D. Data Fitting Results}

All the data were used in the analysis, with the exception of runs which had some known deficiency (e.g. too low a voltage on the driftchamber wire planes). The results for $\xi \mathrm{P}_{\mu} \delta / \rho$ for all targets in the three data-taking runs (to be denoted by Run 1, etc.) are compiled in Appendix B. They are plotted in Fig. V.2, where only statistical errors are shown. The systematic errors will be considered in sections VI and VII. The results incorporate the three corrections to be discussed in section VI. One of the corrections was target-dependent, and its valie for each target is also listed in Appendix B.

It was discovered during Run 2 that the thinner of the two $\mathrm{Cu}$ targets was not sufficiently thick to stop all muons. The amount of material beyond the mean muon stopping range is estimated to be only 2.5 straggling lengths for this target. The fitted $\xi P_{\mu} \delta / \rho$ for this target is significantly lower $(3.00)$ than the combined result from all other targets (see Table V.1), and also lower than the combined result from the thicker $\mathrm{Cu}$ targets $(4.60)$. Since $-0.6 \%$ of the muons which leaked out of the target could have been depolarized in air or In the mylar window of the proportional chamber P3, we have chosen not to include the result for this target in the conputation of the combined result for $\xi P_{\mu} \delta / \rho$.

The values of $\xi \mathrm{P}_{\mu} \delta / \rho$ for each metal, liquid He, and all targets together are tabulated in Table V.1 (the thinner Cu target in Run 2 is excluded; only statistical errors are listed). Let us mention 


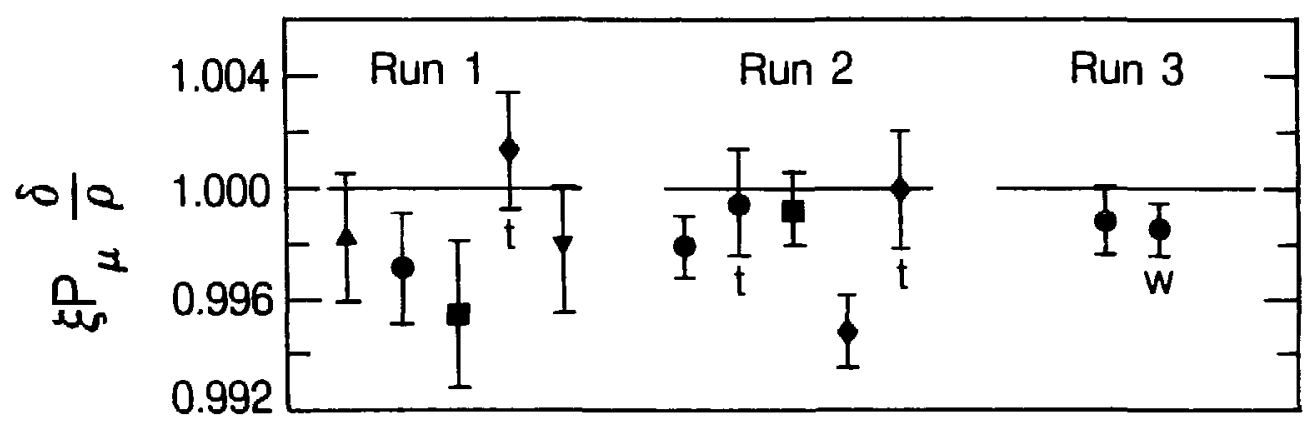

XBL 8512-11755

FIG. V.2. Results for $\xi \mathrm{P}_{\mu} \delta / \rho$ for each target in the three data-taking runs (denoted by Run 1, etc.). Only statistical errors are shown. The targets are Ag (triangles), Al (circles), $\mathrm{Au}$ (squares), Cu (diamonds) and He (inverted triangles). The spin-holding field was $0.3 \mathrm{~T}$ for the target marked by " $\mathrm{W}$ " and $1.1 \mathrm{~T}$ for the other targets. The thicker targets are marked by "t". Target thicknesses are compiled in Appendix A. 
TABLE V.1. Results for $E P_{\mu} \delta / p$ for each metal, liquid He and all targets combined. Only statistical errors are shown.

\begin{tabular}{|c|c|c|}
\hline Material & \multicolumn{2}{|c|}{$\xi P_{\mu} \delta / \rho$} \\
\hline $\mathrm{Ag}$ & 0.9983 & $\begin{array}{l}+0.0022 \\
-0.0023\end{array}$ \\
\hline Al & 0.9985 & $\begin{array}{l}+0.00056 \\
-0.00056\end{array}$ \\
\hline $\mathrm{Au}$ & 0.9986 & $\begin{array}{l}+0.0011 \\
-0.0011\end{array}$ \\
\hline $\mathrm{Cu}$ & 1.0007 & $\begin{array}{l}+0.0015 \\
-0.0015\end{array}$ \\
\hline $\mathrm{He}$ & 0.9979 & $\begin{array}{l}+0.0022 \\
-0.0023\end{array}$ \\
\hline All targets & 0.9986 & $\begin{array}{l}+0.00046 \\
-0.00046\end{array}$ \\
\hline
\end{tabular}


here that in the analysis of the spin-precessed data using the muon spin rotation ( $\mu S R$ ) technique 22 He was found to be $12 \%$ depolarizing. The depolarization was most likely due to muonium formation, and if so, the suppression of muon depolazation in muonium by the $1.1-\mathrm{T}$ longltudinal field would explain the difference between our and $\mu S R$ results. A possible reason for the muonium formation in liquid He was mentioned in section III.C.

The combined results from all targets were computed for the five $\cos \theta_{e}$ bins. In Fig. V. 3 they are plotted as

$$
\xi \mathrm{P}_{\mu}{ }_{\rho}^{\delta} \cos \theta_{\mathrm{av}}=1-\mathrm{r}\left(\theta_{\mathrm{av}}\right)
$$

versus $\cos \theta_{a v}$. They incorporate the corrections to be discussed in section VI. Again, only statistical errors are shown. The fitted straight line in Fig. V.3 indicates the fixed slope extrapolation to $\cos \theta_{\mathrm{av}}=1$

The results for $\xi \mathrm{P}_{\mu} \delta / \rho$ were also evaluated in four muon decay time ranges for the three netal targets ( $\mathrm{Al}, \mathrm{Cu}$ and $\mathrm{Au}$ ) constituting 93\% of the data (here the data from the thinner $\mathrm{Cu}$ target in Run 2 were included). The time ranges were $(0.1-0.85)$ us, $(0.85-2.1) \mu s,(2.1-5.1) \mu s$ and $(5.1-10) \mu s$ after the muon arrival at the stopping target. As mentioned in section III.C, the stopped muon polarization is expected to decay exponentially with characteristic spin-lattice relaxation time constant $T_{1}$ toward the equilibrium situation, where the numbers of muon spins parallel or anti-parallel to the field are almost equal. If $\mathrm{T}_{1}$ is much greater than $10 \mu \mathrm{s}$, the results for $\xi \mathrm{P}_{\mu} \delta / \rho$ from the the four time ranges will have a linear dependence on the quantity 


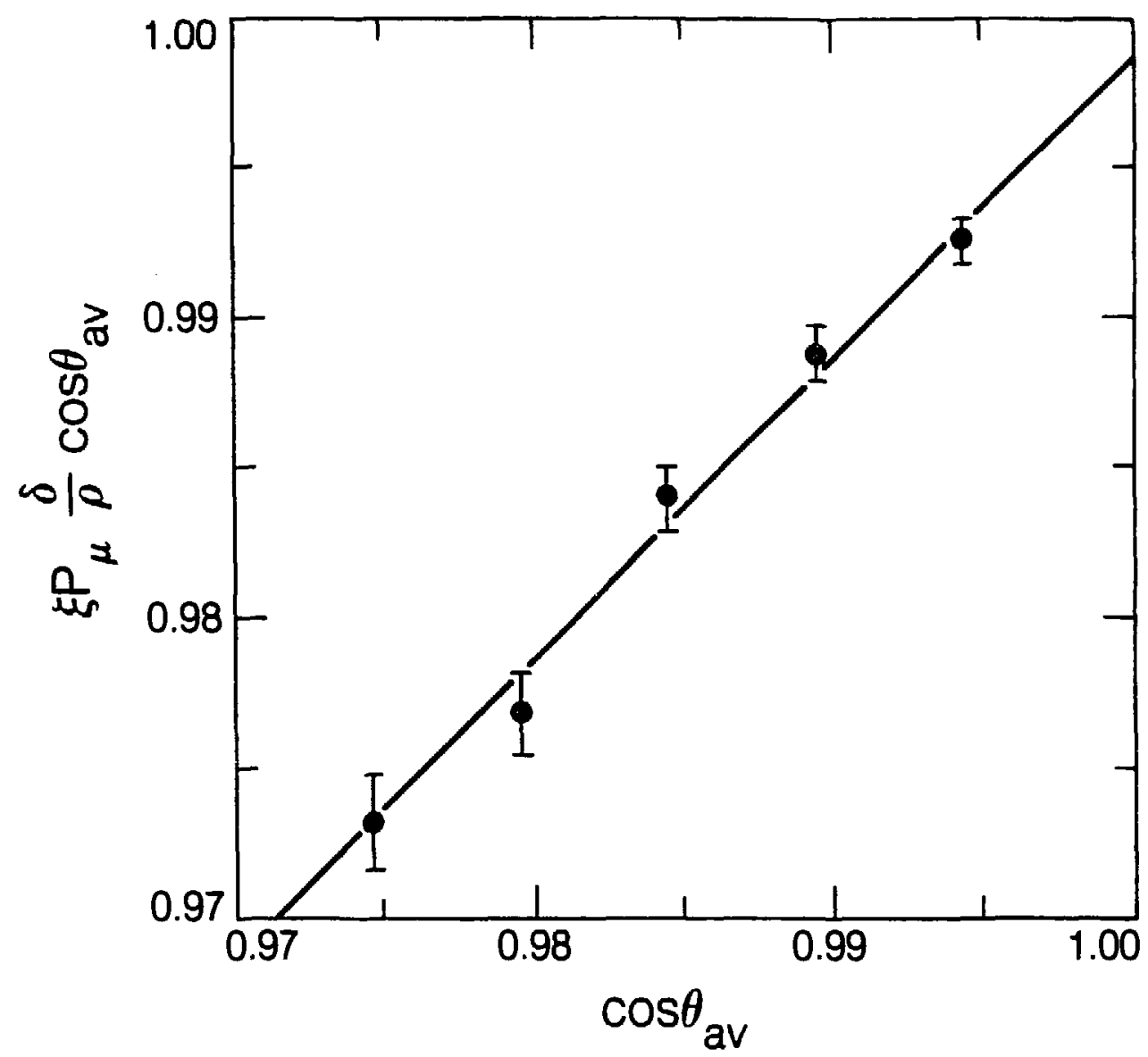

XBL 8512-11758

FIG. V.3. Results for $\xi P_{\mu} \frac{\delta}{\rho} \cos \theta_{a v}$ from the five $\cos \theta_{e}$ bins plotted versus $\cos \theta_{\mathrm{av}}$. Only statistical errors are shown. The corrections, discussed in section VI, have been incorporated. The fitted straight line indicates the fixed slope extrapolation to $\cos \theta_{\mathrm{av}}=1$ 


$$
t=t_{1}+\tau_{\mu}-\left(t_{2}-t_{1}\right)\left\{\exp \left[\left(t_{2}-t_{1}\right) / \tau_{\mu}\right]-1\right\}^{-1}
$$

where $t_{1}$ and $t_{2}$ are the start and end of a time range.

As can be seen in Fig. V. 4 only the results for Al are suggestive of a time-dependent spin relaxation. If the oscillating magnetic fields causing the spin relaxation are produced by the nuclear magnetic dipole moments, the relaxation times for $\mathrm{Al}, \mathrm{Cu}$ and $\mathrm{Au}$ should correlate with their respective dipole moments of $3.6,2.3$ and 0.1 nuclear magnetons. The fitted slope of $(-4.3 \pm 3.4) \cdot 10^{-4} \mu \mathrm{s}^{-1}$ for Al corresponds to $T_{1}$ time of $4.6 \mathrm{~ms}$, which would imply that a correction of $(+9.9 \pm 7.8) \cdot 10^{-4}$ should be added to the result for $\xi \mathrm{P}_{\mu} \delta / \rho$ for $\mathrm{Al}$. Contrary to the expectation based on the result for $\mathrm{Al}$, the best $\mathrm{fit}$ slope for $\mathrm{Cu}$ is actually positive $\left[(+4.5 \pm 4.8) \cdot 10^{-4} \mu \mathrm{s}\right]$. Since our result will be used to set a lower limit on $E_{2} P_{\mu} \delta / \rho$, we have chosen a conservative approach and decided not to apply a correction due to a possible spin relaxation.

Conclusions from the result for $\xi_{\mu} \mathrm{P}_{\mu} \delta / \rho$ will be drawn in section VIII, after we consider the corrections and systematic errors in the next two sections. 


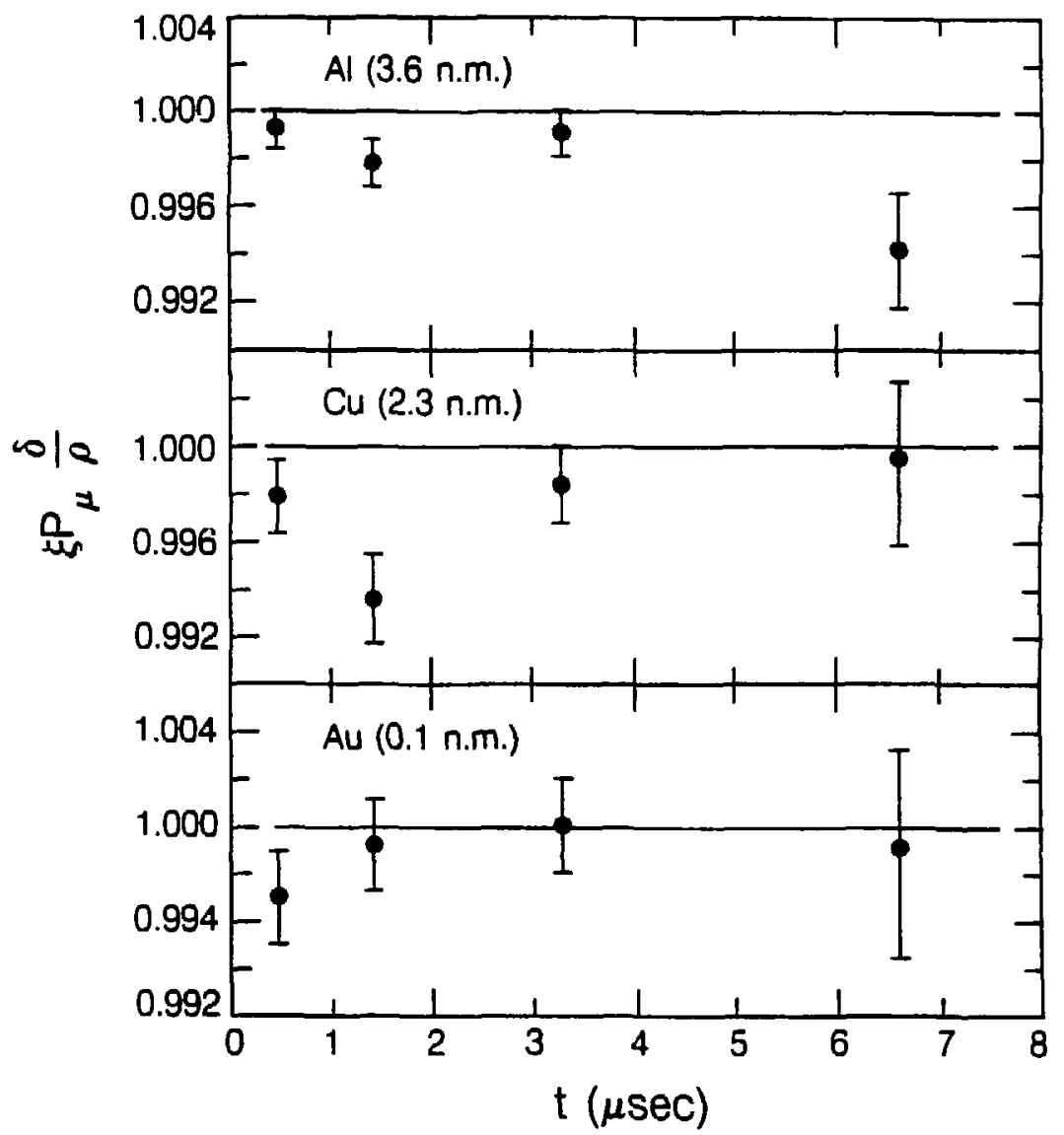

XBL 8512-11757

FIG. V.4. The results for $\xi P_{\mu} \delta / \rho$ evaluated in four muon decay time ranges for the three metal targets, $\mathrm{Al}, \mathrm{Cu}$ and $\mathrm{Au}$, constituting 93\% of the data. Only statistical errors are shown. The results are plotted versus $t$, given by equation (5.12). The target material nuclear magnetic moment in units of nuclear magnetons (n.m.) is Indicated. Only the results for $A l$ are suggestive of a time-dependent spin relaxation. 


\section{CORRECTIONS}

This chapter describes the computation of three corrections. Two corrections were applied to $\cos \theta_{a v}$, computed from data-averaged $\cos \theta_{\mu} \cos \theta_{e}$. Another correction was applied to the result for $\xi \mathrm{P}_{\mu} \delta / \rho$ due to muon depolarization during the stopping process in scattering with unpolarized electrons. The reasons for these corrections have been mentioned in section V.B. We will continue to use the notation introduced in that section.

Let us briefly repeat the reasons for the corrections to $\cos \theta_{\mathrm{av}}$. In the expression (5.4) for the rate at the endpoint $\cos \theta_{a v}$ is defined by $(5.10)$. However, in the analysis $\cos \theta_{a v}$ had to be computed from $\left\langle\cos \theta_{\mu} \cos \theta_{e}\right\rangle$, i.e. $\cos \theta_{\mu} \cos \theta_{e}$ averaged over the spin-held data. Two corrections had to be added to $\left\langle\cos \theta_{\mu} \cos \theta_{e}\right\rangle$. The first correction was due to the difference between $\left\langle\cos \theta_{\mu} \cos \theta_{e}\right\rangle$ computed from the measured tracks, and $\left\langle\cos \theta_{\mu} \cos \theta_{e}\right\rangle$ if it were computed from the true values of $\theta_{\mu}$ and $\theta_{e}$. This correction was mostly due to multiple scattering, although finite chamber resolution also contributed. Since positron scattering depended on the target, this correction was target dependent. The second correction was due to the difference between $\left\langle\cos \theta_{\mu} \cos \theta_{e}\right\rangle$, given by (5.11), and $\cos \theta_{a v}$ defined by $(5.10)$.

The values of the three corrections and the uncertainties in them are listed in Table VI.1. It should be noted that adding a correction to $\cos \theta_{a v}$, i.e. to $\left\langle\cos \theta_{1 \mu} \cos \theta_{e}\right\rangle$, was equivalent to reducing $\xi P_{\mu} \delta / \rho$ by the same amount because $\left\langle\cos \theta_{\mu} \cos \theta_{e}\right\rangle$ was nearly 1 . 
TABLE VI.1. Values of corrections to $\cos \theta_{a v}$ and $\xi P_{\mu} \delta / \rho$ and estimated possible systematic errors in corrections. Adding a correction to $\cos \theta_{a v}$ is equivalent to reducing $\xi P_{\mu} \delta / \rho$ by the same amount.

Correction to $\xi P_{\mu} \delta / \rho$ due to muon depolarization in scattering with $+0.0007<0.0001$ unpolarized electrons Correction (averaged over all targets) to $\cos \theta_{a v}$ due to Coulomb scattering $-0.0001$ $\pm 0.0003$ and finite chamber resolution

Correction to $\cos \theta_{a v}$ due to use of data averaged $\cos \theta_{\mu} \cos \theta_{e}$ 
The possible systematic errors in two of the three corrections listed in Table VI. 1 are estimated to be small compared to $1 \cdot 10^{-4}$.

The three corrections will be considered below in this order: first, the correction to $\cos \theta_{a v}$ due to multiple scattering and finite chamber resolution; second, the correction to $\cos \theta_{\mathrm{av}}$ due to use of (5.11), rather than $(5.10)$; finally, the correction to $\xi P_{\mu} \delta / \rho$ due to muon depolarization in scattering with unpolarized electrons.

VI.A. Correction due to Multiple Scattering and Finite Chamber Resolution

Let us denote $\left\langle\cos \theta_{\mu} \cos \theta_{e}\right\rangle$ computed from the measured tracks by $\left\langle\cos \theta_{\text {meas }}\right\rangle$, and $\left\langle\cos \theta_{\mu} \cos \theta_{e}\right\rangle$ if it were computed from the true values of $\theta_{\mu}$ and $\theta_{e}$ by $\left\langle\cos \theta_{\text {true }}\right\rangle$. The unit vector given by the extrapolation of the measured muon track to the stopping target will be denoted by $\hat{p}_{\mu \text {, meas }}$, and $\theta_{\mu \text {, meas }}$ will denote the corresponding polar angle with respect to the solenoid axis. $\theta_{\mathrm{u}, \text { true }}$ will denote the true polar angle of $-\hat{s}_{\mu}$ at the decay vertex.

Muon depolorization due to scattering with unpolarized electrons, which is cons.. ed in section VI.C, will be ignored. Coulomb scattering is relativisticaliy helicity conserving and non-relativistically spin-conserving. The non-relativistic limit applies to the $\mu^{+}$, which initially have $\beta=0.27$ (when a muon with helicity \pm 1 scatters by an angle $\theta$, muon polarization with respect to the initial direction changes by $\left.=\theta^{2} \beta^{4} / 8\right)$. It can therefore be assumed that the spin polar angle $\theta_{\mu}$, when a muon enters the stopping 


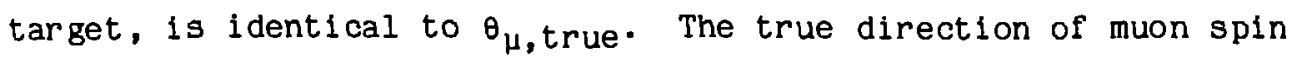
when the muon enters the stopping target will be denoted by $\hat{s}_{\mu, e n t}$. Similarly, let $\hat{\mathrm{p}}_{\mathrm{e}}$, true denote the direction of $\mathrm{e}^{+}$momentum at the decay vertex, and $\theta_{e}$,true the corresponding polar angle. Let the unit vector given by the extrapolation of the measured positron track to the stopping target be denoted by $\hat{\mathrm{p}}_{\mathbf{e}}$, meas, and $\theta_{\mathrm{e}}$, meas be the corresponding polar angle.

We seek the correction $\left\langle\cos \theta_{\text {true }}\right\rangle-\left\langle\cos \theta_{\text {meas }}\right\rangle$. Since $\cos \theta_{\mu}$ and $\cos \theta_{e}$ are both very close to 1 ,

$$
\cos \theta_{\mu} \cos \theta_{e}=\cos \theta_{\mu}+\cos \theta_{e}-1 \text {, }
$$

and hence

$$
\begin{aligned}
\left\langle\cos \theta_{\text {true }}\right\rangle-\left\langle\cos \theta_{\text {meas }}\right\rangle= & {\left[\left\langle\cos \theta_{\mu, \text { true }}\right\rangle-\left\langle\cos \theta_{\mu, \text { meas }}\right\rangle\right]+} \\
& {\left[\left\langle\cos \theta_{e}, \text { true }{ }^{\rangle-\left\langle\cos \theta_{e},\right. \text { meas }}\right\rangle\right] . }
\end{aligned}
$$

Let us consider qualitatively various contributions to the muon average in (6.1), i.e. to the first term on the right-hand side. Similar arguments will apply for positrons. There are several sources of misalignment between $\hat{s}_{\mu \text {, ent }}$ and $\hat{p}_{\mu \text {, meas }}$. Before the muon enters the vacuum window separating the beamline from the muon polarimeter (it is located just in front of the proportional chamber P1; see Fig. III.1), $\hat{s}_{\mu}$ and $\hat{p}_{\mu}$ are misaligned due to scattering in the production target. Muon scattering in the material $\left(-50 \mathrm{mg} / \mathrm{cm}^{2}\right)$ upstream of the stopping target contributes further to the misaligmment. Although scattering of the muons is assumed to be spin-conserving, the muon spin direction does change between the vacuum window and the stopping target dif to spin precession in the longitudinal field. 
In order to estimate the effects of muon scattering in the production target and in the material upstream of the stopping target, let us ignore the magnetic fleld in the stopping target region. It can therefore be assumed that muon spin direction does not change when a muon travels from the vacuum window to the stopping target. Let us denote the true direction of the muon momentum before the muon enters the $P 1$ vacuum window by $\hat{p}_{i}$ (subscript $i$ stands for 'initial'). Let $\theta_{i}$ be the corresponding polar angle. The muon average in (6.1) can be rewritten as

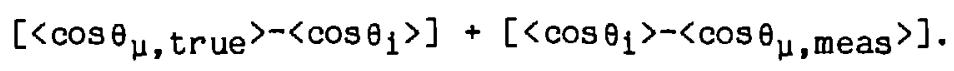

Without a loss of generality, we may assume that at the $\pi^{+}$decay vertex $\hat{s}_{\mu}$ is always anti-parallel to $\hat{p}_{\mu}$. Let us consider the first term in (6.2). The misalignment between $\hat{s}_{\mu \text {, ent (which determines }}$ $\cos \theta_{\mu}$,true since the magnetic field is ignored) and $\hat{p}_{i}$ is due to scattering in the production target. For simplicity, let the scattering angle $\phi_{1}$ in the production target be the same for all muons. The angle $\phi_{1}$ between $\hat{s}_{\mu}$ and $\hat{p}_{\mu}$ is preserved by the magnetic field in the beamline, where muons are transported in vacuum. At the vacuum window $\hat{s}_{\mu}$ is randomly misaligned with respect to $\hat{p}_{i}$ by an angle $\phi_{1}$. By a simple computation

$$
\left\langle\cos \theta_{\mu, \text { true }}\right\rangle=\left\langle\cos \theta_{i}\right\rangle \cos \phi_{1},
$$

and since $\phi_{1}$ is small $(-30 \mathrm{mrad})$ and $\cos \theta_{i}=1$,

$$
\left[\left\langle\cos \theta_{\mu, \text { true }}\right\rangle-\left\langle\cos \theta_{1}\right\rangle\right]=-\phi_{1} 2 / 2 \text {. }
$$

Let us now consider the second term in (6.2). The misalignment between $\hat{p}_{i}$ and $\hat{p}_{\mu, \text { meas }}$ is mostly due to scattering between the vacuum window and the proportional chamber P2, although finite chamber 
resolution and finite accuracy of track extrapolation also contribute. To a good approximation, only material upstream of the mid-point between $P 1$ and $P 2$ contributes to the misalignment $\left(5 \cdot 10^{-4}\right.$ radiation lengths). The acceptance effects and sof tware cuts preferentially reject events with the largest $\mu^{+}$scattering angles between the vacuum window and $P 2$, which leads to a decrease $\left(-6 \cdot 10^{-4}\right)$ in the correction. We will return to these effects below, when we consider the systematic errors in the correction, but for the moment suppose that these effects can be 1 gnored. Again, let the angle $\phi_{2}$ between $\hat{p}_{i}$ and $\hat{p}_{\mu}$, meas be the same for all muons. The unit vectors $\hat{p}_{\mu, \text { meas }}$ are randomly misaligned with respect to $p_{i}$ by an angle $\phi_{2}$. Hence

$$
\left\langle\cos \theta_{\mu, \text { meas }}\right\rangle=\left\langle\cos \theta_{1}\right\rangle \cos \phi_{2}
$$

by the same computation as in $(6.3)$, and

$$
\left[\left\langle\cos \theta_{i}\right\rangle-\left\langle\cos \theta_{\mu, \text { meas }}\right\rangle\right]=\phi_{2} 2 / 2 \text {. }
$$

Therefore the correction for $\mu^{+}$scattering in the material near the stopping target, given by (6.5), has an opposite sign from the correction for $\mu^{+}$scattering in the production target, given by (6.4). Since the correction (6.1) depends on the geometrical acceptance of the apparatus, on cuts in analysis, on angular distribution of the incoming muons, etc., it is not possible to compute it precisely by an analytical computation. Furthermore, the positron average in (6.1) depends on the target so that the correction is also target-dependent. The corrections were therefore computed, to a statistical error of less than $\pm 0.5 \cdot 10^{-4}$ for each stopping target, in a full Monte-Carlo simulation of the polarimeter described in section 
V.C. The scattering model used in the simulation is based on the theory of Nigam, Sundaresan and Wu. ${ }^{32}$ The data generated in the simulation were processed through the same event reconstruction and selection algorithm as the experimental data, and the correction (6.1) was computed from Monte-Carlo events which passed all cuts. The correction varied by less than $2.5 \cdot 10^{-4}$ from $\cos \theta_{e}=0.975$ to $\cos \theta_{e}=1$ for all targets. Hence, the correction was assumed to be the same for all $\cos \theta_{e}$ bins, since it was found that the error in $\xi P_{\mu} \delta / \rho$ due to this assumption was less than $\pm 0.5 \cdot 10^{-4}$. The corrections for all targets are tabulated in Appendix $B$. They include an upward adjustment of $2 \cdot 10^{-4}$ discussed below.

Let us now consider the possible systematic errors in the correction (6.1). The accuracy of the scattering model, assumed co be $\pm 10 \%$ in the scattering angle magnitude, leads to an error of $<1 \cdot 10^{-4}$, but conservatively it is taken to be $\pm 1 \cdot 10^{-4}$. As can be seen from equations (6.4) and (6.5), the value of the correction should vary quadratically with the scattering angle magnitude if acceptance effects can be ignored. This quadratic dependence approximately holds in practice. The error due to the uncertainty in the scattering angle magnitude is relatively small because the correction for muon scattering in the production target has an opposite sign from the correction due to scattering near the stopping target: the muon part of the correction (6.1) is $0.1 \cdot 10^{-4}$ (total), compared to the contribution of scattering in the production target of $-4 \cdot 10^{-4}$. The positron part of the correction, averaged over the stopping targets, is $-1 \cdot 10^{-4}$. 
The correction (6.1) depends on the fraction of events with large scattering angles remaining in the final sample. The number of such events in the final sample is reduced because of software cuts requiring $\cos \theta_{\mu} \geq 0.99$ and a radius at the stopping target $\leq 1.8 \mathrm{~cm}$. Also, such events have a larger beam spot at the stopping target, and consequently there is a smaller probability for a positron to be accepted by the apparatus. The correction (6.1) therefore depends on the distribution of incoming $\mu^{+}$angles, and to a smaller extent on the beam spot at the target.

The true muon distribution in angles and position at the P1 vacuum window is not known since the measured distribution includes the effects of scattering. Let us explain how the measured distribution was obtained. In the experiment, when the data was collected in the spin-precessing mode (i.e. with $70-G$ or $110-G$ vertical field), approximately every thirtieth event was taken with a ' $\mu$-stop' trigger, requiring only a signature of a particle stopping in the target. The angular distribution of the measured muon tracks in such events was used in the Monte-Carlo simulation.

Since essentially all muons at the $P 1$ vacuum window are accepted by the apparatus, it is possible to estimate the true $\mu^{+}$distribution that would yield the measured distribution. When the estimated true $\mu^{+}$distribution was used to compute the correction, the muon part of the correction was $=2 \cdot 10^{-4}$ greater then it was with the measured distribution. Therefore, all corrections computed with the measured distribution were adjusted upwards by $2 \cdot 10^{-4}$, and a possible systematic error of $\pm 2 \cdot 10^{-4}$ was assigned to the correction (6.1) due 
to the uncertainty in the incoming $\mu^{+}$distribution.

Finally, the correction (6.1) depends on the amount of scattering in the production target. The mean thickness of material in the production target traversed by muons is determined by the mean beam momentum, estinated to be $29.2 .5 \mathrm{MeV} / \mathrm{c}$. The $\mu^{+}$momentum from $\pi^{+}$decay at rest is $29.79 \mathrm{MeV} / \mathrm{c}$, which implies that the mean energy lnss in the graphite production target was $0.0092 \mathrm{MeV}$, corresponding to a mean thickness traversed by muons of $6.6 \mathrm{mg} / \mathrm{cm}^{2}$. An uncertainty in the mean $\mu^{+}$beam momentum of $\pm 0.2 \mathrm{MeV} / \mathrm{c}$ implies an uncertainty of $3.8 \mathrm{mg} / \mathrm{cm}^{2}$ in the mean thickness traversed by muons, which leads to a possible systematic error in the correction of $\pm 2 \cdot 10^{-4}$.

When these three systematic errors are added in quadrature, the combined possible syctematic error in the correction ( 6.1 ) becomes $\pm 3 \cdot 10^{-4}$. The correction averaged over all targets was $-1 \cdot 10^{-4}$. 
VI.B. Correction due to Use of Data-Averaged $\cos \theta_{\mu} \cos \theta_{e}$

We seek a correction due to the difference between $\cos \theta_{a y}$ defined by $(5.10)$, and the data-averaged $\cos \theta_{\mu} \cos \theta_{e}$ given by (5.11). The latter will be denoted by $\left\langle\cos \theta_{\mu} \cos \theta_{e}\right\rangle, j$ ust as ${ }^{2} \equiv$ fore. The correction required is given by

$$
\cos \theta_{\mathrm{av}}-\left\langle\cos \theta_{\mu} \cos \theta_{\mathrm{e}}\right\rangle
$$

Here it is assumed that $\left\langle\cos \theta_{\mu} \cos \theta_{e}\right\rangle$ has been computed from the true values of $\theta_{\mu}$ and $\theta_{e}$.

In section V.B it was shown that when statistical fluctuations are ignored, the positron spectrum for the fitted subset of the spin-held data is given by

$$
W(x)-\int\left[a(x)+b(x) \xi P_{\mu} \cos \theta\right] A\left(\theta_{e}, \psi_{e}\right) A(x) R\left(\theta_{\mu}\right) d \omega_{e} d \omega_{\mu}
$$

where $a(x)$ and $b(x)$ are the angle-dependent and angle-independent parts of the muon decay rate; $A\left(\theta_{e}, \psi_{e}\right)$ is the angular acceptance function for positrons, assumed to be independent of the $x$ acceptance function $A(x) ; R\left(\theta_{\mu}\right)$ denotes the $\theta_{\mu}$ distribution of muon spins at the decay vertex for muons reaching the stopping target (the distribution is isotropic in $\psi_{\mu}$ ); $\mathrm{d} \omega$ denotes the integration over the angular phase space, 1.e. $d \omega_{e}=d\left(\cos \theta_{e}\right) d \psi_{e}$; and

$$
\cos \theta=\cos \theta_{\mu} \cos \theta_{e}+\sin \theta_{\mu} \sin \theta_{e} \cos \psi_{\mu} \cos \psi_{e}+\sin \theta_{\mu} \sin \theta_{e} \sin \psi_{\mu} \sin \psi_{e} \text {. }
$$

The two terms in the expression for $\cos \theta$ which contain $\psi_{\mu}$, vanish in (6.7) after integration. Aside from $\cos \theta$, only $A\left(\theta_{e}, \psi_{e}\right)$ in (6.7) depends on $\psi_{e}$. Hence, $W(x)$ can be rewritten in terms of the angular acceptance function which depends only on $\theta_{e}$ :

$$
A\left(\theta_{e}\right)=\int A\left(\theta_{e}, \psi_{e}\right) d \psi_{e} \text {. }
$$


This fact will be useful for the discussion below.

The correction (6.6) depends on the range of $\cos \theta_{e}$ in the fitted data set, and also on the minimum $x$ in the fit of the spin-held data. For $\cos \theta_{e}$ bins 0.005 wide and $x_{\min }=0.97$, the correction was small, only $+1 \cdot 10^{-4}$, and to an accuracy of $\pm 0.1 \cdot 10^{-4}$ it was the same for all $\cos \theta_{e}$ bins. The correction was computed by evaluating the spectrum $W(x)$ numerically, using the estimated true $R\left(\theta_{\mu}\right)$ distribution (see section VI.A), and assuming that $A(x)$ and $A\left(\theta_{e}, \psi_{e}\right)$ were constant.

It was checked that the correction changed negligibly for realistic $A(x)$ and $A\left(\theta_{e}\right)$. The $x$ acceptance varied the most in the smallest $\cos \theta_{e}$ bin (i.e. $0.975 \leq \cos \theta_{e}<0.98$ bin). When the spin-precessed data were combined from all targets and fitted according to (5.1), with $A(x)$ given by $(5.2)$, it was found that the $x$ acceptance in this $\cos \theta_{e}$ bin fell by $17 \%$ at $x=.93$ and by $1.5 \%$ at $x=1$, relative to the acceptance maximum at $x=0.985$. The correction (6.6) changed by less than $0.1 \cdot 10^{-4}$ when this $A(x)$ was used in the computation of $W(x)$.

The angular acceptance $A\left(\theta_{e}\right)$ for the spin-held data should be similar to $A\left(\theta_{e}\right)$ for the spin-precessed data. The latter is given by the number of events as a function of $\cos \theta_{e}$, since after timing cuts the spin-precessed data is equivalent to the unpolarized, i.e. $P_{\mu}=0$, data, and the angular distribution of decay positrons for $P_{\mu}=0$ data is isotropic. The angular acceptance at $\cos \theta_{e}=0.975 \mathrm{fell}$ by $-20 \%$ relative to $\cos \theta_{e}=1$. The correction (6.6) changed by less than $0.1 \cdot 10^{-4}$ for a $10 \%$ variation in $A\left(\theta_{e}\right)$ in a single $\cos \theta_{e}$ bin. 
Since $A\left(\theta_{e}\right)$ depends on $x$, one may define $\cos \theta_{a v}$ using the angular acceptance function for the center of the fitted $\cos \theta_{e}$ bin. If such acceptance function is denoted by $A_{c}\left(\theta_{e}\right)$, then

$$
\cos \theta_{a v}=\frac{\int \cos \theta_{\mu} \cos \theta_{e} A_{c}\left(\theta_{e}\right) R\left(\theta_{\mu}\right) d\left(\cos \theta_{e}\right) d \omega_{\mu}}{\int A_{c}\left(\theta_{e}\right) R\left(\theta_{\mu}\right) d\left(\cos \theta_{e}\right) d \omega_{\mu}} .
$$

With this definition of $\cos \theta_{a v}$ the correction (6.6) is still accurate to $\pm 0.1 \cdot 10^{-4}$ for a realistic variation of $A\left(\theta_{e}\right)$ with $x$.

VI.C. Muon Depolarization in Scattering with Unpolarized Electrons

Let us denote by the unit vector $\hat{o}_{\mu}$ the direction the muon spin would have if scattering with electrons were absent. Muon polarization with respect to $\hat{o}_{\mu}$ is reduced due to spin-exchange effects in scattering with unpolarized electrons. The polarization with respect to $\hat{o}_{\mu}$ would have been preserved in the absence of scattering. For simplicity, let us assume that at the $\pi^{+}$decay vertex the muon spin is anti-parallel to $\hat{p}_{\mu}$, as it is for a pure $V-A$ interaction. Let the solenold axis define the z-direction. Muon depolarization with respect to $\hat{o}_{\mu}$ in scattering with unpolarized electrons during the stopping process will be denoted by $\Delta P$. We will continue to assume that Coulomb scattering from nuclei is spin-conserving. The expectation value of the muon spin $\hat{s}_{\mu}$ with respect to $\hat{o}_{\mu}$ is

$$
\left\langle\hat{s}_{\mu}\right\rangle_{\mu}=(\hbar / 2)(1-\Delta P),
$$

Which implies that the expectation value with respect to the z-axis is 


$$
\left\langle\hat{s}_{\mu}\right\rangle_{z}=(1-\Delta P\rangle\left\langle\hat{o}_{\mu}\right\rangle_{z} \text {. }
$$

In section VI.A the correction (6.1), due to the difference between $\left\langle\cos \theta_{\mu} \cos \theta_{e}\right\rangle$ computed from the measured tracks and $\left\langle\cos \theta_{\mu} \cos \theta_{e}\right\rangle$ computed from the true values of $\theta_{\mu}$ and $\theta_{e}$, has been found assuming that $\cos \theta_{\mu}$, true is given by the $z$-component of $\hat{o}_{\mu}$, rather than by $\left\langle\hat{s}_{\mu}\right\rangle_{z}$. Hence, after the correction (6.1) has been added to $\left\langle\cos \theta_{\mu} \cos \theta_{e}\right\rangle$, the resulting value must in addition be multiplied by $(1-\Delta P)$. Since $\Delta P$ is only $7 \cdot 10^{-4}$, as will be shown below, and $\left\langle\cos \theta_{\mu} \cos \theta_{e}\right\rangle$ is $>0.97$, an upward correction equal to $\Delta \mathrm{P}$ has been made directly to the result for $\xi P_{\mu} \delta / \rho$.

We now consider the computation of $\Delta \mathrm{P}$. It was shown by Ford and Mullin ${ }^{2 \theta}$ that for a non-relativistic $\mu^{+}$which scatters with unpolarized $e^{-}$through a center of mass angle $\theta$, the probability that the final $\mu^{+}$spin direction is parallel $(\varepsilon=1)$ or anti-parallel $(\varepsilon=-1)$ to the initial spin is

$$
Q(\varepsilon, \theta)=\frac{1+\varepsilon}{2}-\varepsilon \frac{m^{2}}{\mu^{2}} \beta^{4}\left[\sin ^{2}(\theta / 2)-\sin ^{4}(\theta / 2)+\sin ^{6}(\theta / 2)\right]
$$

where $\beta$ is the muon velocity in the lab frame and $m=m_{e}$ and $\mu=m_{\mu}$. If the muons are initially fully polarized, the polarization along the direction $\hat{o}_{\mu}$ after one scatter is

$$
\left.P_{\mu}=1-2 \frac{m^{2}}{\mu^{2}} \beta^{4}\left[\sin ^{2}(\theta / 2)-\sin ^{4}(\theta / 2)+\sin ^{6}(\theta / 2)\right]\right\}
$$

and similarly, if the polarization is initially $P_{\mu, i}$, the polarization $P_{\mu, f}$ after one additional scatter is 


$$
P_{\mu, f}=P_{\mu, 1}\left\{1-2 \frac{m^{2}}{\mu^{2}} \beta^{4}\left[\sin ^{2}(\theta / 2)-\sin ^{4}(\theta / 2)+\sin ^{6}(\theta / 2)\right]\right\} .
$$

The corresponding fractional energy loss is

$$
w=\frac{m}{\mu} \beta^{2} \sin ^{2}(\theta / 2)
$$

One can write a differential equation for the depolarization as a furiction of energy. For the energy loss $d E=w E$, where $E$ is the muon kinetic energy $E=(\gamma-1) \mu$, the rate of depolarization is

$$
\frac{d P_{\mu}}{d E}=P_{\mu}(E)\left\{2 \frac{m}{\mu^{2}} \frac{B^{2}}{(\gamma-1)}\left[1-\sin ^{2}(\theta / 2)+\sin ^{4}(\theta / 2)\right]\right\} .
$$

The non-relativistic cross-section is Inversely proportional to $\sin ^{4}(\theta / 2)$, and hence the $\sin (\theta / 2)$ terms in $(6.7)$ can be 1 gnored. The polarization $P_{\mu}(E)$ will be $>.999$, and can be set equal to unity on the right-hand side. Surface muons, coming from $\pi^{+}$decay at rest, Initially have kinetic energy $E_{0}=4.1 \mathrm{MeV}$. Since muon energy loss for E>3 KeV is almost entirely due to scattering with electrons (see section III.C), $\Delta \mathrm{P}$ is given by

$$
\begin{aligned}
\Delta P=\int_{3 \mathrm{keV}}^{E_{0}} 2 \frac{\mathrm{m}}{\mu^{2}} & \frac{\beta^{2}}{(\gamma-1)} d E=2 \frac{\mathrm{m}}{\mu^{2}} \int_{0}^{E_{0}}\left[2-3 \frac{E}{\mu}\right] \mathrm{dE}= \\
& =4 \frac{\mathrm{mE} 0}{\mu^{2}}\left(1-\frac{3}{4} \frac{E_{0}}{\mu}\right)=7 \cdot 3 \cdot 10^{-4} .
\end{aligned}
$$

It should be noted that $\Delta P$ is relativily insensitive to the lower limit in the integration. The uncertainty in this correction is therefore estimated to be small compared to $10^{-4}$.

The values of all corrections and the uncertainties in them have been listed in Table VI. 1. 


\section{SYSTEMATIC ERRORS}

The possible sources of systematic errors $\geq 1 \cdot 10^{-4}$ are summarized in Table VII.1. The systematic error in the scattering correction has been discussed in section VI.A. The sources of the remaining systematic errors will be considered one at a time below. The systematic errors listed in Table VII.1 should in principle be uncorrelated; they add in quadrature to $\pm 7.5 \cdot 10^{-4}$.

At the end of this section we will also consider the systematic error due to events with decay positrons produced by an extra muon in the stopping target, rather than the muon causing the trigger. This error will be shown to be small compared to $1 \cdot 10^{-4}$.

\section{VII.A. $\quad x=1$ Calibration}

Recall that the $x=1$ calibration was determined from the $x=1$ edge position in the spin-precessed data (see section V.A). The position of the $x=1$ point on the reconstructed momentum scale was a free parameter in the first stage of fitting, when the spin-precessed data were fitted alone, and in the second stage, when the spin-held and spin-precessed data were fitted simultaneously. The position of $x=1$ was assumed to be identical for the two data sets. However, the $x=1$ calibration points may difter because of the strong longitudinal field in the target region for the spin-held data, which provided extra bending for positron trajectories. Hence, for the same $x$, positron trajectories in the two data sets had a different 
TABLE VII.1. The possible sources of systematic error $\geq 1 \cdot 10^{-4}$ and their estimated contributions.

Difference in $x=1$ calibration $( \pm .00035)$ and

$x$ acceptance $( \pm .0002)$ between the spin-held and spin-precessed data $\pm 0.00055$

Spectrometer momentum dispersion $\pm 0.0001$

Uncertainty in the longitudinal position of wire planes for chambers near the target $\pm 0.00025$

Accuracy of $\cos \theta_{\mu}$ and $\cos \theta_{e}$ reconstruction using first-order optics approximation $\pm 0.00025$

Total 10 possible error $\pm 0.00075$ 
distribution in the spectrometer magnet, and there could be a small difference in $x=1$ calibration points due to imperfections in the momentum reconstruction algorithm. Let us denote by $x_{1, s-h}$ the position of $x=1$ for the spin-held data. Similarly, let $\mathrm{r}_{1, \mathrm{~s}-\mathrm{p}}$ denote the position of $x=1$ for the spin-precessed data.

The possible difference in $\mathrm{x}=1$ calibration

$$
\Delta \mathrm{x}_{1}=\mathrm{x}_{1, \mathrm{~s}-\mathrm{r}}-\mathrm{x}_{1, \mathrm{~s}-\mathrm{p}}
$$

was estimated by fitting the data. We have chosen not to rely on the results from the Monte-Carlo simulation, since the simulation did not use the measured spectrometer field. The field in the simulation was computed assuming a cylindrical symmetry and hence it was only approximate.

The position of $x=1$ for the spin-precessed data, i.e. $x_{1, s-p}$, was already known from the first stage of fitting. The position of $x=1$ for the spin-held data, i.e. $x_{1, s-h}$, was determined by fitting the spin-held data according to (5.3), just as before, but leaving the position of $x=1$ as a free parameter. In order to increase the statistical power of the spin-held data to determine the position of $x=1$, the cut to eliminate cloud muons (described in section III.B) was not applied.

The mean $\Delta x_{1}$ averaged over all $f i v e \cos \theta_{e}$ bins and all targets was found to be $(0.17 \pm 0.45) \cdot 10^{-4}\left(x^{2}=36\right.$ for 54 degrees of freedom $)$. The slope in $\Delta x_{1}$ versus $\cos \theta_{e}$ was consistent with zero $(0.0010 \pm 0.0066)$, and hence $\Delta x_{1}$ was assumed to be the same for all $\cos \theta_{e}$ bins. It was found empirically that for a non-zero $\Delta x_{1}$ the error in $\xi P_{\mu} \delta / \rho$ was $8 \Delta x_{1}$. This error was determined by shifting the 
spin-held data in $x$ and observing the resulting change in $\xi P_{\mu} \delta / \rho$. Since our result will be used to set a lower limit on $\xi P_{\mu} \delta / \rho$, a conservative approach was chosen and an upwards correction to $\xi P_{\mu} \delta / \rho$ due to a positive central value of $\Delta x_{1}$ was not made. Hence, just a systematic error of $\pm 3 \cdot 5 \cdot 10^{-4}$ was assigned to $\xi P_{\mu} \delta / \rho$.

\section{VII.B. Momentum acceptance}

The acceptance in reduced positron momentum $x$, which was assumed to be the same for the spin-held and spin-precessed data, ri:?y also differ because of the strong longitudinal field in the target region. Let us introduce a function $D(x)$ to describe the possible difference in $x$ acceptance between the two data sets. Since the normalization was a free parameter in the fit, it may be assumed that $D(1)=1$. The lowest order, 1.e. linear, term in $D(x)$ may be estimated by extending the minimum $x$ for the spin-held data from $x=0.97$ down to $x=0.93$, and adding $D(x)$ to the fitting function for the spin-held data (5.3). The fitting function for the spin-held data then becomes

$$
N_{2}\left\{\left[1-r\left(\theta_{a v}\right)\right] S_{V-A}(x, 1)+r\left(\theta_{a v}\right) S_{V-A}(x, 0)\right\} A(x) D(x)
$$

where $N_{2}$ is a free normalization; $S_{V-A}(x, 1)$ and $S_{V-A}(x, 0)$ are the pure $V-A$ spectra at $P_{\mu} \cos \theta=1$ and $P_{\mu} \cos \theta=0$ respectively; $r\left(\theta_{a v}\right)$ is the relative rate at the end-point; $A(x)$ is the quadratic acceptance function (5.2); and $D(x)$ describes the difference in acceptance between the spin-held and spin-precessed data:

$$
D(x)=1+D_{1}(x-1)
$$

where $D_{1}$ is a free parameter. 
The mean $D_{1}$, averaged over the $f i v e \cos \theta_{e}$ bins and all targets, was found to be $0.14 \pm 0.18\left(x^{2}=44\right.$ for 54 degrees of freedom $)$. The slope in $D_{1}$ versus $\cos \theta_{e}$ was consistent with zero $(7 \pm 27)$, and hence $D_{\uparrow}$ was assumed to be the same for all $\cos \theta_{e}$ bins. It was empirically determined that when $D(x)$ was used in the fit of the spin-held data, the result for $E_{\mu} P_{\mu} \delta / \rho$ changed by $0.001 \cdot D_{1}$ relative to its value with $D_{1}=0$. The rate of change in $\xi_{\mu} \delta / \rho$ with $D_{1}$ varied quadratically with $\left(1-x_{\min }\right)$, where $x_{\min }$ is the minimum $x$ in the fit of the spin-held data. The $x_{\min }$ of 0.97 was optimal for the smallest combined (statistical and systematic) error.

A conservative approach was chosen here, $j$ ust as for the $x=1$ calibration error, and an upward correction to $\xi \mathrm{P}_{\mu} \delta / \rho$ due to a positive central value of $D_{1}$ was not made. A systematic error of $\pm 2 \cdot 10^{-4}$ was assigned to $\xi P_{\mu} \delta / \rho$ due to a possible difference in $x$ acceptance between the spin-held and spin-precessed data.

Since the possible differences in the $x=1$ calibration and $x$ acceptance are both due to the longitudinal field in the target region, these two errors were combined linearly in the computation of the total systematic error. 


\section{VII.C. Momentum Dispersion}

The spectrometer momentum dispersion was measured to be $(1.056 \pm 0.008) \% / \mathrm{cm}$ (see section IV). Let us estimate the change in $\xi \mathrm{P}_{\mu} \delta / \rho$ if the dispersion is decreased, for example. A decrease in the dispersion means that the reconstructed reduced positron momentum $x$ is shifted closer to $x=1$. Hence one may think of the change in dispersion in terms of a factor $\lambda(\lambda<1)$ by which the reconstructed momentum scale is compressed towards $x=1$.

The spin-held data is given approximately by a linear function vanishing at $x=1$ plus a constant. It can be therefore easily shown that when the $x$ scale is compressed by a factor $\lambda$, the normalization parameter $\mathrm{N}_{2}$ in the fitting function for the spin-held data (equation 5.3) increases by a factor of $=1 / \lambda^{2}$. Near $x=1$ the number of events per $\mathrm{x}$ bin increases by a factor of $1 / \lambda$. Since this number is proportional to $\mathrm{N}_{2} r\left(\theta_{a v}\right)$, where $r\left(\theta_{a v}\right)$ is the relative rate at the end-point, $r\left(\theta_{a v}\right)$ decreases by a factor of $\lambda$, as was verified in the analysis. Recall that $\xi_{\mu} \mathrm{P}_{\mu} \delta / \rho$ was extracted from $r\left(\theta_{a v}\right)=1-\left(\xi P_{\mu} \delta / \rho\right) \cos \theta_{a v}$. The mean $r\left(\theta_{a v}\right)$ was 0.015 , and hence a possible systematic error of $\pm 1 \cdot 10^{-4}$ was assigned to $\xi \mathrm{P}_{\mu} \delta / \rho$ due to an uncertainty in the spectrometer momentum dispersion. 
VII.D. Reconstruction of $\cos \theta_{\mu}$ and $\cos \theta_{e}$

There are two more sources of possible systematic errors in the reconstruction of $\cos \theta_{\mu}$ and $\cos \theta_{e}$, in addition to the systematic error in the scattering correction to $\left\langle\cos E_{\mu} \cos \theta_{e}\right\rangle$, which was discussed in section VI.A. Here we will first consider the systematic errors due to the uncertainties in the longitudinal positions of the wire planes in the proportional chambers near the target. Then we will consider a systematic error due to fitting curved tracks in the first-order optics approximation for cylindrically symmetric fields, described in section IV.

Let us denote the difference between $\left\langle\cos \theta_{\mu}\right\rangle$ (i.e. the data-averaged $\cos \theta_{\mu}$ ) if it were computed from the true angles and $\left\langle\cos \theta_{\mu}\right\rangle$ computed from the measured angles by $\Delta \cos \theta_{\mu} \cdot \operatorname{similarly}$, $\Delta \cos \theta_{e}$ will denote the difference for positrons. An uncertainty of $\pm 2 \mathrm{~mm}$ in the longitudinal distance between the proportional chambers P1 and P2 (see Fig. III.1) leads to a systematic error of $\pm 2 \cdot 10^{-4}$ in $\Delta \cos \theta_{\mu}$, and consequently in $\xi \mathrm{P}_{\mu} \delta / \mathrm{\rho}$. An urcertainty of $\pm 2 \mathrm{~mm}$ in the longitudinal position of the proportional chamber $\mathrm{P} 3$ with respect to the drift chambers D1 and D2 leads to a systematic error of $\pm 1.5 \cdot 10^{-4}$ in $\Delta \cos \theta_{e}$. These errors are combined in quadrature to yield $\pm 2.5 \cdot 10^{-4}$.

In the first-order optics approximation particle trajectories are determined by means of transfer matrices, computed from the magnetic field values on the solenoid axis and the magnetic rigidity. In the region from the proportional chamber P1 to the driftchamber D2 the 
magnetic field is given by the sum of the 'upstream' fleld, produced by the two coils surrounding the target, and the 'downstream' field, produced by the coils in the solenoid bore. The two sets of coils were powered independently.

In track reconstruction we have used the axial field computed by the POISSON program. A comparison was made between the POISSON axial field and the measured axial field (the measurements were taken at different magnet currents than those used in the experiment). For the comparison the upstream POISSON field was scaled so that there was an agreement in the axial field at the target, and the downstream POISSON field was scaled to obtain agreement in the axial field at the last wire plane in driftchamber D2. After the normalization, the POISSON axial field agreed with the measured field to within $12 \%$. The measured field was always lower than the POISSON field, with the largast difference being near the field minimum in driftchamber D1 and in the upstream field tail near P1.

The optimal scaling factors for the upstream and downstream fields for track reconstruction were determined by minimizing the average $x^{2}$ for the curved positron tracks. When the scaling factors were determined in an identical manner for the data from the full Monte-Carlo simulation of the polarimeter, it was found that in the absence of scattering and chamber resolution effects $\Delta \cos \theta_{\mu}$ was $+2 \cdot 10^{-6}$ and $\Delta \cos \theta_{e}$ was $-2 \cdot 10^{-5}$. Hence, the accuracy of the first-order optics approximation in determining $\cos \theta_{\mu}$ and $\cos \theta_{e}$ is assumed to be less than $\pm 1 \cdot 10^{-4}$. The systematic errors in $\Delta \cos \theta_{\mu}$ and $\Delta \cos \theta_{e}$ are assigned due to the uncertainties in determining the 
scaling factors, and due to the difference between the POISSON and measured axial fields.

The scaling factors were determined to an accuracy of less than 5\%. To allow for the difference between the POISSON and measured axial flelds, the systematic errors in $\Delta \cos \theta_{\mu}$ and $\Delta \cos \theta_{e}$ are assumed conservatively to correspond to a $10 \%$ change in the scaling factors. For a $10 \%$ change in the scaling factor for the upstream field, $\Delta \cos \theta_{\mu}$ changed by $8 \cdot 10^{-5}$ and $\Delta \cos \theta_{e}$ by $5 \cdot 10^{-5}$. For a $10 \%$ change in the scaling factor for the downstream field, $\Delta \cos \theta_{e}$ changed by $1 \cdot 10^{-4}$. Hence a possible systematic error of $2.5 \cdot 10^{-4}$ is assigned to $\xi \mathrm{P}_{\mu} \delta / \rho$ due to an uncertainty in the accuracy of $\cos \theta_{\mu}$ and $\cos \theta_{e}$ reconstruction using the first-order optics approximation.

\section{VI.D. Extra Muons}

A small fraction of events in the final sample have decay positrons produced by an extra muon in the stopping target, rather than the muon causing the trigger. This fraction was smaller than .0013 as will be shown below. The extra muons have a lower polarization $P_{\mu}$ due to cloud muon contamination (see section III.B), and a smaller average $\cos \theta_{\mu}$. When cloud muons are included, the average muon beam polarization is reduced to $99 \%$. The mean $\cos \theta_{\mu}$ for the muon beam is 0.9966 compared to 0.9970 for muons which pass the $\cos \theta_{\mu}>0.39$ cut. Hence, a very small upward correction of $0.1 \cdot 10^{-4}$ could have bsen made to $\xi \mathrm{P}_{\mu} \delta / \rho$ due to a smaller mean $\cos \theta_{\mu}$ and lower $P_{\mu} f u_{i}$ extra muons. Since the correction was so tiny we have chosen 
to ignore it.

Let us now compute the probability for an event to have the decay positron produced by an extra muon in the stopping target, rather than the muon causing the trigger. The muon causing the trigger will be referred to as the primary muon.

An extra muon may arrive either before or after the primary muon. First, consider extra muons arriving before the primary muon. Recall that the trigger consisted of two parts: a muon part, requiring a signature of a particle stopping in the target, followed in delayed coincidence of $0.1-10 \mu \mathrm{s}$ by a positron part, requiring a particle registered by detectors downstream of the target. Let us denote by $p_{t}$ the probability that an arriving muon will satisfy the requirement for the muon part of the trigger in the absence of deadtime. The probability $\mathrm{p}_{t}$ is estimated to be greater than $90 \%$. Let $\lambda$ denote the $\mu^{+}$beam rate. It can be shown that to a very good approximation for $\lambda \tau_{\mu} \ll<$ the probability that there was another muon in the target when the primary muon had arrived is given by

$$
\left(1-p_{t}\right) \lambda \tau_{\mu}+p_{t} \lambda^{2} \tau_{\mu}^{2}
$$

where $\tau_{\mu}$ is muon lifetime. The second term in the expression above is due to muons arriving in the 10 us deadtime after each registered muon stop. For the average muon flux of $15 \mathrm{kHz}$, the probability that there was another muon in the target. when the primary muon had arrived is therefore at the most 0.0043 .

Consider now extra muons arriving after the primary muon. If an extra particle was detected upstream of the target after the primary muon 'had stopped, the event was cut in analysis if the 'extra-after' 
particle was detected before the decay positron. The efficlency for detection of extra muons is estimated to be close to $100 \%$, except for those arriving in the 300 ns notch after the primary muon. The notch, in which the extra particles were not detected, was necessary because of the after-pulsing in the proportional chambers P1 and P2, which resulted in false extra-after signals. For the average $15 \mathrm{kHz}$ muon flux the probability of an extra muon arriving after the primary muon in the $300 \mathrm{~ns}$ noteh is 0.0045 .

If the decay positron was produced by an extra muon, there is only a 15\% chance that such event will pass the cut requiring an agreement in track extrapolation at the stopping target. Therefore, the probability that the decay positron was produced by an extra muon (which arrived either before or after the primary muon) is at the most 0.0013 , as mentioned above. 
VIII. DISCUSSION AND CONCLUSIONS

VIII. A. Limit on $\xi P_{\mu} \delta / \rho$

We quote our result as a lower limit for $\xi \mathrm{P}_{\mu} \delta / \rho$ since we are unable to correct for all possible sources of muon depolarization in the stopping target. Because our goal was to set a lower limit, we did not apply the possible upward corrections to $\xi P_{\mu} \delta / \rho$ discussed in sections V.D, VII.A and VII.B. In the absence of these corrections the combined result from all stopping targets

$$
\xi P_{\mu} \delta / p=0.99863 \pm 0.00046 \text { (stat.) } \pm 0.00075 \text { (syst.) }
$$

is still consistent $(1.60)$ with the V-A prediction of 1 . With the unphysical region $\left(\xi \mathrm{P}_{\mu} \delta / \rho>1\right)$ excluded it implies the $90 \%$ confidence limit

$$
E P_{\mu} \delta / \rho>0.99747 \text {. }
$$

The result is in agreement with the $90 \%$ confidence limit

$$
\xi P_{\mu} \delta / p>0.9951
$$

obtained in the analysis of the spin-precessed data using the muon spin rotation ( $\mu S R$ ) technique. ${ }^{11,12}$ The combined result from the two methods of analysis implies the $90 \%$ confidence limit

$$
\xi P_{\mu} \delta / \rho>0.99753 \text {. }
$$

The two methods of analysis have different major sources of possible systematic error, and $i t$ is therefore appropriate to combine the two results as independent measurements. The limit from the two results will be used to draw conclusions below.

So $f a r$ it has been assumed that both left-handed and right-handed 
neutrinos $\nu_{\mu}$ and $\nu_{e}$ are massless or sufficiently light not to affect the kinematics $\left(m_{v}<1 \mathrm{MeV} / \mathrm{c}^{2}\right)$. We will continue to work in this 1 imit until section VIII.G, where larger masses will be considered.

VIII.B. Limits on Right-Handed Currents with Massless Neutrinos

In the model with 'manifest' left-right symmetry (see section I) and massless neutrinos

$$
\xi P_{\mu} \delta / \rho=1-2\left(2 \varepsilon^{2}+2 \varepsilon \zeta+\zeta^{2}\right)
$$

to lowest order in the mass-squared ratio $\varepsilon$ and the mixing angle $\zeta$ for the gauge bosons $W_{1}$ and $W_{2}$. The lower limit on $\xi P_{\mu} \delta / \rho$ therefore constrains both $\varepsilon$ and $\zeta$. The contour corresponding to the $90 \%$ confidence limit on $\varepsilon$ and $\zeta$ is plotted in Fig. I.1. Assuming $m\left(W_{1}\right)=81 \mathrm{GeV} / \mathrm{c}^{2}, 90 \%$ confidence l1mits for the following special cases are obtained: $m\left(W_{2}\right)>514 \mathrm{GeV} / c^{2}$ for $\zeta=0$ and $m\left(W_{2}\right)>432 \mathrm{GeV} / \mathrm{c}^{2}$ when $\zeta$ is unconstrained; $|\zeta|<0.035$ for $m\left(W_{2}\right)=\infty$ and $-0.050<\zeta<0.035$ when $m\left(W_{2}\right)$ is unconstrained.

Herczeg ${ }^{3}$ has obtained the expression $\mathrm{fc}_{\mathrm{c}}\left(1-\xi \mathrm{P}_{\mu} \delta / \rho\right)$ for a general left-right symmetric (LRS) theory, assuming that neutrinos are either sufficiently light that their effect on the spectrum can be ignored, or that they are too heavy to be produced in muon decay. In addition to $\varepsilon$ and $\zeta$, the general expression, which we do not reproduce here, contains the left-handed and $r i g h t-h a n d e d$ weak coupling constants, the left-handed and right-handed quark mixing angles, the CP-violating phase in $W_{1}$ and $W_{2}$ mixing, and the mixing angles and CP-violating phases for neutrinos. For a general LRS 
theory, constraints that are obtained from the limit on $\xi P_{\mu} \delta / \rho$ depend on the assumptions one makes about the values of these parameters.

VIII.C. Limits on $m\left(v_{\mu L}\right)$ and $v_{\mu L}$ Helicity in $\pi^{+}$Decay

The limit on $\xi P_{\mu} \delta / \rho$ implies limits on the mass of the left-handed muon neutrino and its helicity in pion decay. The weakest limits are obtained if it is assumed that right-handed currents are absent. In that case $\xi \delta / \rho=1$ and hence $P_{\mu}>0.9975$. The $90 \%$ confidence limit on the $v_{\mu L}$ helicity in $\pi^{+}$decay is therefore $h\left(v_{\mu L}\right)<-0.9975$. The corresponding limit on the $\nu_{\mu L}$ veiocity $(\beta=v / c>0.9975)$ in $\pi^{*}$ decay implies the $90 \%$ confidence limit $\mathrm{m}\left(\nu_{\mu L}\right)<2.1 \mathrm{MeV} / \mathrm{c}^{2}$. For comparison the world average value $21 \mathrm{~m}\left(v_{\mu L}\right)<0.5 \mathrm{MeV} / \mathrm{c}^{2}$ implies $P_{\mu}>0.99986$ in the absence of right-handed currents.

VIII.D. Restrictions on the Lorentz Structure of Leptonic Charged Weak Interactions

Mursula and Scheck ${ }^{2}$ have recently obtained limits on non- $(V-A)$ weak couplings using a helicity projection form of the flavor retention contact interaction Hamiltonian:

$$
\begin{aligned}
& H=\left(G_{0} / \sqrt{2}\right)\left\{h_{11}(s+p) e v_{e}(s+p)_{\nu_{\mu} \mu}+h_{12}(s+p)(s-p)+h_{21}(s-p)(s+p)\right. \\
& +h_{22}(s-p)(s-p)+g_{11}\left(v^{\alpha+a^{\alpha}}\right)\left(v_{\alpha}+a_{\alpha}\right)+g_{12}\left(v^{\alpha+a} \alpha\right)\left(v_{\alpha}-a_{\alpha}\right) \\
& +g_{21}\left(v^{\alpha} a^{\alpha}\right)\left(v_{\alpha}+a_{\alpha}\right)+g_{22}\left(v^{\alpha} a^{\alpha}\right)\left(v_{\alpha}-a_{\alpha}\right) \\
& \left.+f_{11}\left(t^{\alpha \beta}+t^{\prime \alpha \beta}\right)\left(t_{\alpha \beta}+t^{\prime}{ }_{\alpha \beta}\right)+f_{22}\left(t^{\alpha \beta-t^{\prime}}{ }^{\alpha \beta}\right)\left(t_{\alpha \beta}-t^{\prime}{ }_{\alpha \beta}\right)+h_{n} c .\right)
\end{aligned}
$$


where $s_{1 k}=\bar{\psi}_{i} 1 \psi_{k}, p_{i k}=\bar{\psi}_{i} \gamma_{5} \psi_{k}, v_{1 k}^{\alpha}=\bar{\psi}_{i} \gamma^{\alpha} \psi_{k}, a_{1 k}^{\alpha}=\bar{\psi}_{1} \gamma^{\alpha} \gamma_{s} \psi_{k}$, $t_{i k}^{\alpha \beta}=\bar{\psi}_{i}\left(0^{\alpha \beta} / \sqrt{2}\right) \psi_{k}, t \cdot \underset{i k}{\alpha \beta}=\bar{\psi}_{1}\left(o^{\alpha \beta} \gamma_{5} / \sqrt{2}\right) \psi_{k}$ and the particle indices are indicated in the $h_{11}$ term.

There are several advantages to using a Hamiltonian of this form. For a pure $(V-A)$ interaction only $g_{22}$ is non-zero $\left(g_{22}=1\right)$ while all other coupling constants vanish. Also, in the limit of massless particles the combinations of covariants in each term of equation (8.1) project onto states of definite helicity. As a consequence, the number of interference terms in any decay rate is minimal since only scalar-pseudoscalar and tensor terms interfere.

Several constraints are imposed on coupling constants in the models ${ }^{2}$ with 'factorization and universality', where it is assumed that: (i) the charged weak interactions are mediated by a single heavy boson with spin 0,1 , or 2 , (ii) V, A, T couplings are $\mathrm{e}^{-} \mu$ universal, but (iii) scalar and pseudoscalar coupling constants can be proportional to the charged lepton mass:

$$
\begin{aligned}
& h_{12}, h_{21} \text { real, positive semi-definite } \\
& h_{22}=h_{12} * \text { with }\left|h_{11}\right|^{2}=h_{12} h_{21} \\
& g_{11}, g_{22} \text { real, positive semi-definite } \\
& g_{21}=g_{12} * \text { with }\left|g_{12}\right|^{2}=g_{11} g_{22} \\
& f_{22}=f_{11} *
\end{aligned}
$$

In terms of the coupling constants in equation (8.1) the deviation of $\xi \delta / \rho$ from the $V-A$ value of 1 is given by

$$
1-\xi_{\delta} / \rho=\frac{8\left|g_{11}\right|^{2}+2\left|h_{21}\right|^{2}+2\left|h_{11}-2 f_{12}\right|^{2}}{4\left(\left|g_{11}\right|^{2}+\left|g_{22}\right|^{2}\right)+\left|h_{12}\right|^{2}+\left|h_{21}\right|^{2}+\left|h_{12}-2 f_{11}\right|^{2}+\left|h_{22}-2 f_{22}\right|^{2}}
$$


Hence the measurement of ${ }_{5} \mathrm{P}_{\mu} \delta / \rho$ sets limits on $g_{11}, h_{11}, h_{2_{1}}$, and $f_{12}$. The weakest limits are obtained if it is assumed that the couplings responsible for $\mu^{+}$decay are unrelated to $\pi^{+}$decay couplings. In this case the value of $P_{\mu}$ cannot be expressed in terms of the couplings in (8.1), and hence the limits are obtained from $\xi \delta / \rho \leq \xi \mathrm{P}_{\mu} \delta / \rho$. If only one coupling other than the $V-A$ coupling $g_{22}=1$ is non-zero the $90 \%$ conf 1 dence limit $\xi \delta / \rho>0.9975$ restricts $\left|g_{11}\right|$ or $\left|f_{12}\right|<0.035$ and $\left|h_{11}\right|$ or $\left|h_{21}\right|<0.070$. Stronger limits on the couplings can be obtained with assumptions of weak interaction universality. In the model with manifest left-right symmetry and no left-right mixing, for example, $P_{\mu}=1-2\left|g_{11}\right|^{2} /\left|g_{22}\right|^{2}$ to lowest order in $g_{11}$, and hence a $90 \%$ confidence limit on the $V+A$ coupling $g_{11}$ is $\left|g_{11}\right|<0.025$. The limits on $g_{11}, f_{11}, h_{11}$, and $h_{21}$ in turn provide constraints on other couplings $\left(e . g . f_{22}, h_{22}\right.$ ) in models with factorization and universality, which were discussed discussed in the paragraph above. These additional constraints are due to relations (8.2).

Although the limit on $\xi \mathrm{P}_{\mu} \delta / \rho$ constrains the pure $\mathrm{V}+\mathrm{A}$ coupling $g_{11}$, it does not constrain the couplings $g_{12}$ and $g_{21}$, corresponding to the $V-A$ coupling at one vertex (eve or $\left.\mu v_{\mu}\right)$, but $V+A$ coupling at the other vertex. The couplings $g_{12}$ and $g_{21}$ are constrained by the muon decay parameter $p$

$$
\rho-3 / 4=-(12 / A)\left\{\left|g_{12}\right|^{2}+\left|g_{21}\right|^{2}+2\left|f_{11}\right|^{2}+2\left|f_{22}\right|^{2}+\operatorname{Re}\left(h_{11} f_{11}{ }^{*}+h_{22} f_{22}{ }^{*}\right)\right\}
$$

where

$$
\begin{aligned}
A=4\left\{4 \left(\left|g_{22}\right|^{2}\right.\right. & \left.+\left|g_{11}\right|^{2}+\left|g_{12}\right|^{2}+\left|g_{21}\right|^{2}\right)+\left|h_{11}\right|^{2}+\left|h_{12}\right|^{2}+\left|h_{21}\right|^{2}+\left|h_{22}\right|^{2} \\
& \left.+12\left(\left|f_{11}\right|^{2}+\left|f_{22}\right|^{2}\right)\right\} .
\end{aligned}
$$


Assuming that only $\mathrm{B}_{12}$ and $\mathrm{B}_{21}$ couplings are non-zero in addition to the $V-A$ coupling $g_{22}=1$, the world average value ${ }^{2 l}$ of $\rho=0.7517 \pm 0.0026$ implies the $90 \%$ confidence limit

$$
\left\{\left|g_{12}\right|^{2}+\left|g_{21}\right|^{2}\right\}<0.0045
$$

when the unphysical region $[(\rho-3 / 4)>0]$ is excluded.

\section{VIII.E. Limits on Composite Leptons}

The possibility that leptons and quarks are composite at some mass scale $\Lambda$ has received considerable attention in recent years. Among the strongest experimental limits on $\Lambda$ currently quoted ${ }^{34}$ are those from the deviation of muon gyromagnetic ratio $\mathrm{g}$ from $2(>860$ $\mathrm{GeV})$, v-hadron scattering (>2.5 TeV), Bhabba scattering ( 1 to $3 \mathrm{TeV})$, and the reaction $\mathrm{e}^{+} \mathrm{e}^{-} \rightarrow \mu^{+} \mu^{-}(2-3.5 \mathrm{TeV})$.

The effects of compositeness may be analyzed in terms of new effective contact interactions. Following the analyses of Peskin, ${ }^{35}$ and Lane and Barany ${ }^{36}$ the most general $S U(2) \times U(1)$ invariant contact interaction contributing to $\mu \rightarrow$ eviv is

$$
\begin{aligned}
& L_{\text {cont }}=\left(g^{2} / \Lambda^{2}\right)\left[n_{1}\left(\bar{v}_{\mu L} \gamma^{k} \mu_{L}\right)\left(\bar{e}_{L} \gamma_{k} v_{e L}\right)+n_{2}\left(\bar{v}_{\mu R} \gamma^{k} \mu_{R}\right)\left(\bar{e}_{R} \gamma_{k} v_{e R}\right)\right. \\
& +\eta_{3}\left(\bar{\nu}_{\mu L} \gamma^{k} \nu_{e L}\right)\left(\bar{e}_{R} \gamma_{k} \mu_{R}\right)+\eta_{4}\left(\bar{e}_{L} \gamma^{k} \mu_{L}\right)\left(\bar{\nu}_{\mu R} \gamma_{k} v_{e R}\right) \\
& +\eta_{3}\left(\bar{v}_{\mu L} \mu_{R}\right)\left(\bar{e}_{L} v_{e R}\right)+\eta_{6}\left(\bar{v}_{\mu L} \nu_{e R}\right)\left(\bar{e}_{L} \mu_{R}\right) \\
& \left.+\eta_{7}\left(\bar{v}_{\mu R} \mu_{L}\right)\left(\bar{e}_{R} v_{e L}\right)+\eta_{B}\left(\bar{v}_{\mu R} v_{e L}\right)\left(\bar{e}_{R} \mu_{L}\right)\right]
\end{aligned}
$$

where $g$ is a coupling of hadronic strength; the $\eta_{i}$ are usually assumed to be of order unity and are normalized so that $\left|\mathrm{n}_{\mathrm{L}}\right|=1$ in the diagonal coupling 


$$
\left(g^{2} / 2 \Lambda^{2}\right)\left[n_{L}\left(\bar{e}_{L} \gamma^{k} e_{L}\right)\left(\bar{e}_{L} \gamma_{K} e_{L}\right)+\ldots\right] \cdot
$$

The first and second terms in equation (8.3) are purely left-handed and $\mathrm{right-handed} \mathrm{respectively,} \mathrm{and} \mathrm{hence} \mathrm{are}$ indistinguishable from the usual $\mathrm{V}-\mathrm{A}$ and $\mathrm{V}+\mathrm{A}$ interactions.

There are three special cases of interest:

1. If only left-handed (right-handed) leptons are composite then only the purely left-handed (right-handed) term survives, i.e. only $n_{1}\left(\eta_{2}\right) \neq 0$.

2. If both left-handed and right-handed leptons are composite but contain quite different sets of constituents then the purely left-handed and right-handed terms dominate, i.e. $n_{1}, n_{2}$ >>other $n_{i}$. 3. If there is no $v_{R}$, or $m\left(v_{R}\right)$ is large, onl $y n_{1}, n_{3} \neq C$. Assuming an effective interaction Lagrangian $L_{\text {eff }}=L_{V-A}+L_{\text {cont }}$ we obtain the endpoint decay rate:

$$
1-\xi \mathrm{P}_{\mu} \delta / \rho=2(620 \mathrm{GeV} / \Lambda)^{4}\left(g^{2} / 4 \pi\right)^{2}\left(n_{2}^{2}+\eta_{3}^{2}+\eta_{5}^{2} / 4\right) \text {. }
$$

The limit $\xi \mathrm{P}_{\mu} \delta / \rho>0.9975$ then implies

$$
\Lambda^{2}>(3310 \mathrm{GeV})^{2}\left(\mathrm{~g}^{2} / 4 \pi\right)\left(\eta_{2}^{2}+\eta_{3}^{2}+\eta_{5}^{2} / 4\right)^{\frac{1}{2}}
$$

with $90 \%$ confidence. (If the not unreasonable assumptions $g^{2 / 4 \pi}=2.1$ and $n_{1}>0.2$ are made, the limit $\Lambda>2600 \mathrm{GeV}$ would be obtained.)

For the special cases discussed earlier the limit becomes

1. Only left-handed leptons composite: no limit. Only right-handed leptons composite: $\quad \Lambda^{2}>(3310 \mathrm{GeV})^{2}\left(g^{2} / 4 \pi\right) n_{2}$

2. Left- and right-handed leptons have different sets of constituents: $\Lambda^{2}>(3310 \mathrm{GeV})^{2}\left(g^{2} / 4 \pi\right) n_{2}$ 3. No $v_{R}$, or $M\left(v_{R}\right)$ large: $\Lambda^{2}>(3310 \mathrm{GeV})^{2}\left(g^{2} / 4 \pi\right) n_{3}$ 


\section{VIII.F. Limits on Familons}

It has been suggested by Wilczek ${ }^{7}$ that the absence of large CP-invariance violations in strong interaction could be explained by a group of genuine flavor symmetries of the Lagrangian. Spontaneous breakdown of family symmetries leads to characteristic neutral massless Nambu-Goldstone bosons, which he called 'familons'. The coupling of familons at low energies is described by effective Lagrangians of the type

$$
\Delta L=F^{-1} j_{\lambda} \partial \lambda_{f}
$$

where $F$ is the energy scale at which the flavor symmetry is spontaneously broken and $j_{\lambda}$ is the appropriate current. Arguments based on cosmology suggest that $10^{9}<\mathrm{F} \leq 10^{12} \mathrm{GeV}$.

Familons may be detected by observing rare decay modes, in particular muon decay $\mu^{+}+\mathrm{e}^{+}+\mathrm{f}$ with the branching ratio given by

$$
b_{\text {ef }}=\frac{\Gamma(\mu \rightarrow e f)}{\Gamma(\mu \rightarrow e v \bar{\nu})}=\frac{2.5 \cdot 10^{14} \mathrm{GeV}^{2}}{F_{\mu e}^{2}} .
$$

Since the decay $\mu \rightarrow e f$ is isotropic and familon is massless, the existence of familons would be indicated by a spike at the endpoint in the spin-held data (see Fig. V.1). The absence of such a spike allows us to obtain a limit on the branching ratio bef (8.4) and accordingly on the energy scale $F_{\mu e}$ at which the flavor symmetry is broken.

The fitting procedure for the computation of the branching ratio $b_{\text {ef }}$ was essentially identical to that described in section V.A. Let us just mention that the decay rate $\mu \rightarrow e f$ was added to the fitting 
function (5.3) for the spin-held data, and that this rate could be neglected in the fitting function for the spin-precessed data. The mean branching ratio, averaged over the $f i v e \cos \theta_{e}$ bins and all stopping targets was found to be $(0.3 \pm 1.1) \cdot 10^{-6}$. The systematic effects discussed in section VII contribute a possible systematic error of $\pm 0.9 \cdot 10^{-6}$. The largest contribution $\left( \pm 0.8 \cdot 10^{-6}\right)$ is due to a possible difference in $x=1$ calibration betweer the spin-held and spin-precessed data sets. If the unphysical region (bef $<0$ ) is i gnored $90 \%$ confidence limits

$$
b_{e f}<2.6 \cdot 10^{-6}
$$

and

$$
F_{\mu \mathrm{e}}>9.9 \cdot 10^{9} \mathrm{GeV}
$$

are obtained.

VIII.G. Limits on $m\left(W_{2}\right)$ with $m\left(v_{\mu R}\right) \neq 0$

It was mentioned in section I that if neutrinos are Majorana particles the left-handed and $r i g h t-h a n d e d$ neutrinos may have different masses. So far only massless or very light neutrinos have been considered. In the most general case the limit on $m\left(W_{2}\right)$ that can be extracted from the data depends on the masses of the right-handed neuirinos, on neutrino mixing angles, and on the mixing angle $\zeta$ for $W_{1}$ and $W_{2}$. Here we present approximate limits on $m\left(W_{2}\right)$ for a particular case of massive Majorana neutrinos: we will assume a massive $v_{\mu R}$ with $m\left(\nu_{\mu R}\right) \leqslant 16.5 \mathrm{MeV} / \mathrm{c}^{2}$, and a very $\mathrm{light} \nu_{\mathrm{eR}}$, $m\left(\nu_{e R}\right)<1 \mathrm{MeV} / \mathrm{c}^{2}$. Manifest left-right symmetry will be assumed just 
as before. For simplicity we will also assume that there is no mixing between $w_{1}$ and $w_{2}$, i.e. $\zeta=0$ and $w_{2} w_{R}$, and that there is no mixing between neutrinos. The analysis method used in the computation of the limits is described in Appendix C. The limits on $\mathrm{m}\left(\mathrm{W}_{2}\right)$ are only approximate because of the lack of radiative corrections for the spectrum with massive neutrinos (they have not been computed), and also because of several simplifications in the treatment of systematic errors. The limits on $m\left(W_{2}\right)$ are expected to be accurate to $-2 \%$.

The approximate limits on $m\left(W_{2}\right)$ versus $m\left(v_{\mu R}\right)$, computed assuming $m\left(W_{1}\right)=81 \mathrm{GeV} / \mathrm{c}^{2}$, are shown in Fig. VIII.1. The allowed region is above the contour. It can be seen from Fig. VIII. 1 that the limits on $m\left(W_{2}\right)$ are essentially the same for $m\left(v_{\mu R}\right) \leq 1 \mathrm{MeV} / \mathrm{c}^{2}$, but begin to drop rapidly below that value for $m\left(\nu_{\mu R}\right)>6 \mathrm{MeV} / \mathrm{c}^{2}$. The valley at $m\left(v_{\mu R}\right)=9 \mathrm{MeV} / \mathrm{c}^{2}$ corresponds to a 2.50 deviation of $m\left(W_{2}\right)$ from $\infty$. For $m\left(\nu_{\mu R}\right) \geq 17 \mathrm{MeV} / \mathrm{c}^{2}$ the limits on $m\left(W_{2}\right)$ decrease dramatically since the endpoint of the $V+A$ spectrum spectrum with a massive $v_{\mu R}$ approaches the minimum $x=0.97$ used in the $f$ it of the spin-held data. The small discrepancy between the limit for $m\left(v_{\mu R}\right)=0$ in Fig. VIII. 1 and the value quoted earlier in section VIII.B is partly due to a different treatment of the systematic errors, and partly due to a small difference $(0.0002)$ in the results from fitting of all the data in one $\cos \theta_{e}$ bin and from fitting the data separately for each target in five $\cos \theta_{e}$ bins. 


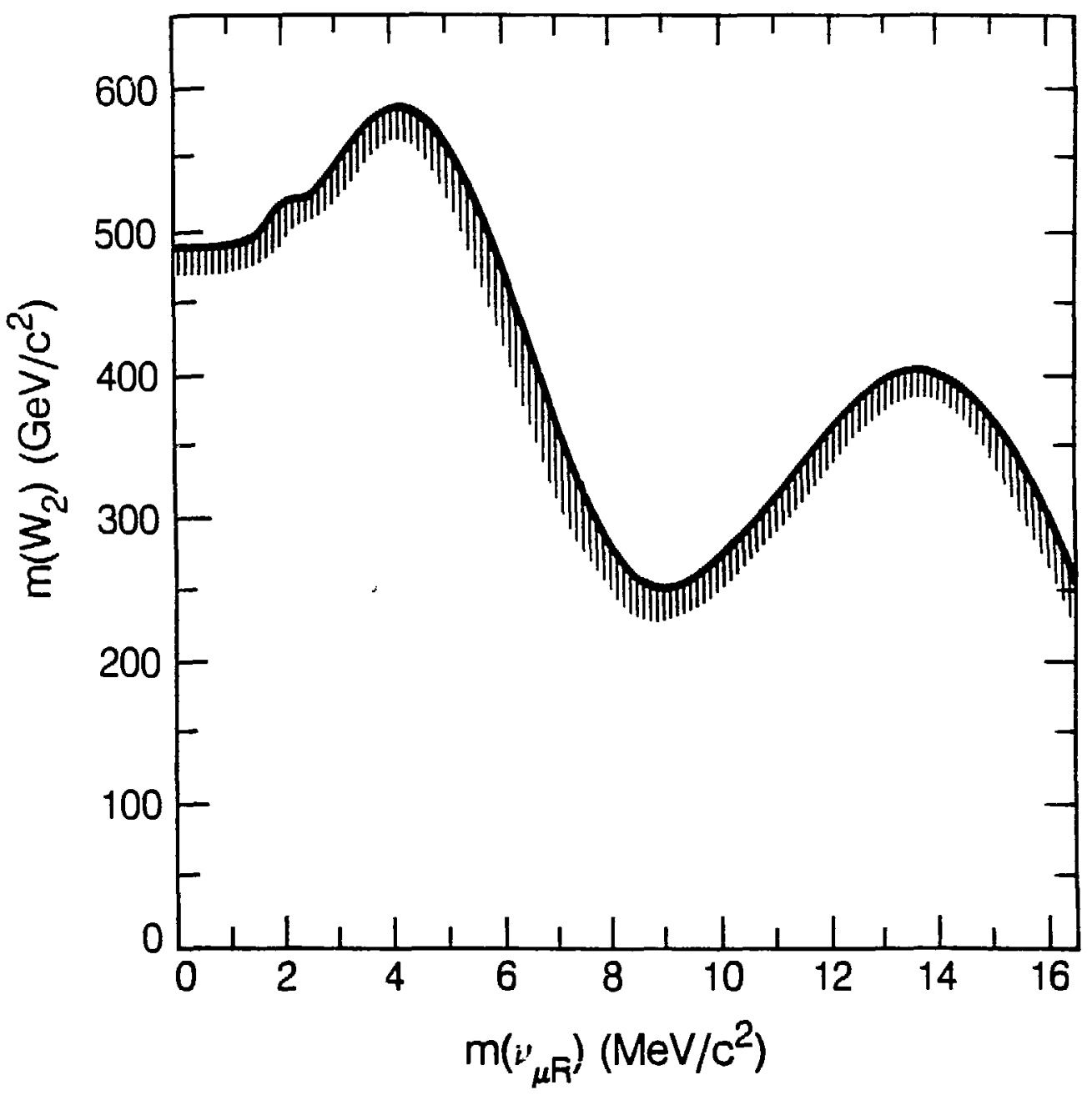

YBL 8512-11761

EIG. VIII.1. Approximate limits on $m\left(W_{2}\right)$ versus $m\left(v_{\mu R}\right)$ assuming $m\left(v_{e R}\right)<1 \mathrm{Mev} / \mathrm{c}^{2}$, nc mixing for neutrinos and no mixing for the gauge bosons $W_{1}$ and $W_{2}$. The allowed region 1 ies above the contour. The computation of the limits is described in the text. 
VIII.H. Concluding Remarks

To summarize, we have made a precise measurement of the endpoint $\mathrm{e}^{+}$decay rate opposite to the $\mu^{+}$spin. The main conclusion is that the result of the measurement is consistent with the V-A model of weak interactions. We have used the result to deduce limits on the mass of the predominantly $r i g h t$-handed boson $W_{2}$ and the left-right mixing angle $\zeta$ in the left-right symmetric models; the $\nu_{\mu L}$ mass and helicity in $\pi^{+}$decay; non-(V-A) couplings in helicity projection form; the mass scale of composite leptons; and the branching ratio for $\mu+e+f$, where $f$ (familon) is the neutral massless Nambu-Goldstone boson associated with flavor symmetry breaking.

Let us briefly consider the reduction in the error on $\xi P_{\mu} \delta / \rho$ that would be necessary to improve the linit on $m\left(W_{2}\right)$ by a factor of -2 . Assuming a central value of $\xi P_{\mu} \delta / \rho=1$, the $90 \%$ confidence 1 imit $m\left(W_{2}\right)>800 \mathrm{GeV} / \mathrm{c}^{2}$ (with the left-right mixing angle $\zeta$ unconstrained) would require a combined statistical and systematic 10 error of $\pm 1 \cdot 1 \cdot 10^{-4}$. In view of several sources of possible systematic errors $>0^{-4}$, which were discussed in sections VI.A and VII, such accuracy would be very difficult to achieve with our experimental method.

In the absence of novel experimental techniques, the discovery of $W_{2}$, assuming that its mass is in the $\mathrm{TeV}$ region, will have to await the advent of high energy supercolliders. Elchten et $a{ }^{3}{ }^{3}$ have considered the prospects for a discovery of $W_{2}$, or of an associated neutral gauge boson $Z_{2}$, at $p p$ or $\bar{p}$ machines. As a discovery criterion they have adopted the requirement that 1000 gauge bosons 
are produced in the rapidity interval $|y|<1.5$. It was assumed that $W_{2}$ and $Z_{2}$ have similar branching ratios for leptonic decay and the same gauge couplings as $W_{1}$ and $Z_{1}$. For a $\bar{p}$ collider with a center of mass energy of $40 \mathrm{TeV}$, for example, the maximum $\mathrm{W}_{2}$ masses of 2.4 , 4.7 and $8.0 \mathrm{TeV} / \mathrm{c}^{2}$ were obtained for integrated - iminosities of $10^{38}$, $10^{39}$ and $10^{40} \mathrm{~cm}^{-2}$. For the same luminosities the maximum $\mathrm{Z}_{2}$ masses are $1.9,3.8$ and $7.1 \mathrm{TeV} / \mathrm{c}^{2}$. The conclusion for the present moment, however, is that all experimental results are consistent with a standard SU(2) L $\times U(1)$ electroweak model. 


\section{APPENDIX A: STOPPING TARGETS}

The muons were stopped in high-purity ( $\geq 99.99 \%)$ metal foils or in liquid He. Since folls of optimum thickness were unavallable, the stopping targets were made either of two back-to-back foils, or of a single foil preceded and followed by $1 \mathrm{mil}$ aluminum foils.

The stopping target thicknesses are tabulated in Table A.1. The compositions of targets having $1 \mathrm{mil}$ Al foils are listed in upstream to downstream order. The mean amount of target material encountered by decay positrons is listed as 'residual thickness'. The residual thickness is also tabulated in terms of calculated $\mu^{+}$rms range straggling lengths. The effect of the $1 \% \Delta \mathrm{p} / \mathrm{p}$ momentum bite has been included. Comparison of the calculated $r$ anges with an experimental range curve taken in kun 2 (i.e. the second data-taking run) indicates that the error on the number of the residual straggling lengths is unlikely to exceed \pm 0.5 .

The $\mathrm{Ag}$ and He targets were used only in Run 1. The residual thicknesses and straggling lengths for the other targets apply only to Run 2. For Runs 1 and 3 the residual thicknesses for $A l, A u$ and Cu varied by small amounts from the Run 2 values due to use of magic gas (30\% isobutane, $69.7 \%$ argon, $0.3 \%$ freon) in proportional chambers for Run 2 and the presence of an additional proportional chamber upstream of the target in Run 3. Let us mention here another minor difference between Runs 1,2, and 3. During Run 1 the solenoid bore was filled with methane-8\% methylal gas; during Run 2 a He bag was placed in the region between the driftchambers D2 and D3 (see 
Fig. III.1); during Run 3 the He bag was removed and the solenoid bore was exposed to air. 
TABLE A.1. The composition and thickness of the muon stopping targets. Superscript ' *' denotes thicker targets.

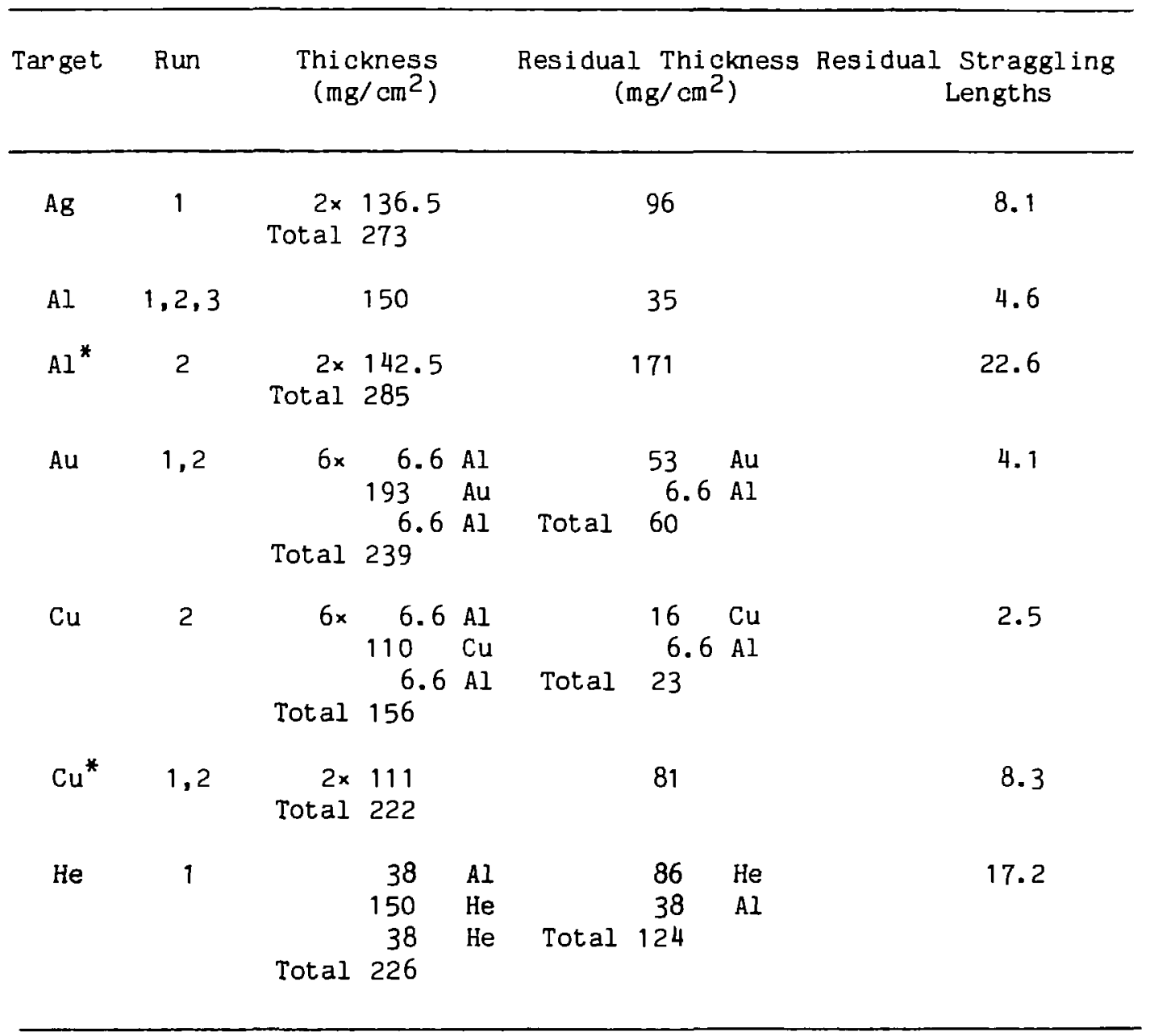


APPENDIX B: TABLE OF DATA FITTING RESULTS

The results for $\xi P_{\mu} \delta / \rho$ are tabulated in Table $B .1$ for each target in the three data-taking runs, denoted by Run 1 , etc.. Only statistical errors are shown. The systematic errors are discussed in sections VI and VII. The results incorporate the four corrections discussed in section VI. Table B. 1 also lists the values of the target-dependent multiple Coulomb scattering correction to $\cos \theta_{\mathrm{av}}$, discussed in section VI.A. Adding a correction to $\cos \theta_{a v}$ is equivalent to reducing $\xi \mathrm{P}_{\mu} \delta / \rho$ by the same amount. Target thicknesses have been compiled in Appendix A. The thicker targets are denoted by a supersoript * 
TABLE B.1. Results for $\xi \mathrm{P}_{\mu} \delta / \rho$ for each target in the three data-taking runs denoted by Run 1, etc.. Only statistical errors are shown. The last column lists the target-dependent Coulomb scattering correction discussed in section VI.A.

\begin{tabular}{|c|c|c|c|c|}
\hline Run & Tar get & \multicolumn{2}{|c|}{$\xi P_{\mu} \delta / \rho$} & Scattering \\
\hline 1 & $\mathrm{Ag}$ & 0.9983 & $\begin{array}{l}+0.0022 \\
-0.0023\end{array}$ & -0.0007 \\
\hline 1 & AI & 0.9972 & $\begin{array}{l}+0.0019 \\
-0.0020\end{array}$ & -0.0001 \\
\hline 1 & $\mathrm{Au}$ & 0.9956 & $\begin{array}{l}+0.0027 \\
-0.0028\end{array}$ & -0.0004 \\
\hline 1 & $\mathrm{Cu}^{*}$ & 1.0014 & $\begin{array}{l}+0.0021 \\
-0.0021\end{array}$ & -0.0004 \\
\hline 1 & $\mathrm{He}$ & 0.9979 & $\begin{array}{l}+0.0022 \\
-0.0023\end{array}$ & -0.0001 \\
\hline 2 & AI & 0.9979 & $\begin{array}{l}+0.0011 \\
-0.0011\end{array}$ & -0.0001 \\
\hline 2 & $\mathrm{AI}^{*}$ & 0.9995 & $\begin{array}{l}+0.0019 \\
-0.0019\end{array}$ & -0.0004 \\
\hline
\end{tabular}

Table B. 1 


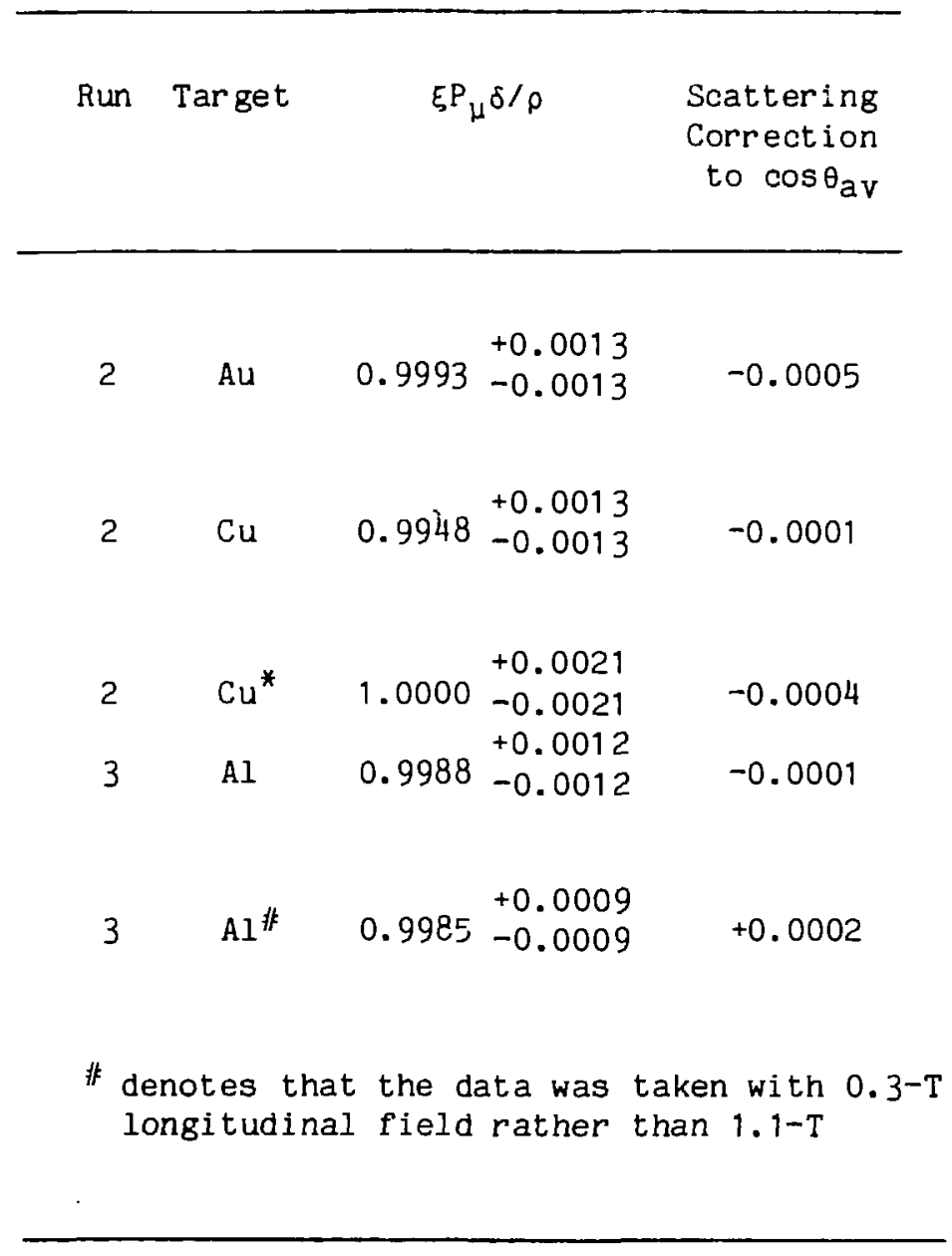

Table B. 1 (cont.) 
APPENDIX C: COMPUTATION OF LIMITS ON $m\left(W_{2}\right)$ with $m\left(v_{\mu R}\right) \neq 0$

We descri'e the analysis method used in the computation of limits on $m\left(W_{2}\right)$ as a function of $m\left(\nu_{\mu R}\right)$ for $m\left(\nu_{\mu R}\right) \leq 16.5 \mathrm{MeV} / \mathrm{c}^{2}$. We assume that $v_{e R}$ is very light, $m\left(v_{e R}\right)<1 \mathrm{MeV} / \mathrm{c}^{2}$, that there is no mixing between $W_{1}$ and $W_{2}$, i.e. $\zeta=0$ and $W_{2} \equiv W_{R}$, and that there is no mixing between neutrinos.

The muon decay rate for massive neutrinos has been calculated by Shrock. ${ }^{39}$ If radiative corrections, positron mass, and $\nu_{e R}$ mass are neglected, the $\mathrm{V}+\mathrm{A}$ differential decay rate is given by

$\frac{d^{2} \Gamma}{d x d(\cos \theta)} \sim\left(1-v^{2} / k^{2}\right)^{2} x^{2}\left\{(3-2 x)+(3-x) \nu^{2} / k^{2}-\cos \theta\left[1-2 x-(1+x) \nu^{2} / k^{2}\right]\right\}$

where $\nu=m\left(\nu_{\mu R}\right), k^{2}=m_{\mu}^{2}-2 m_{\mu} E_{e}$, and $x=E_{e} / E_{0}$. Here $E_{0}$ is the maximum positron energy for the spectrum with massless neutrinos (i.e. $E_{0}$ is equal to $E_{\max }$ in section $\left.I I\right)$. The maximum positron energy for a spectrum with a massive $m\left(\nu_{\mu R}\right)$ is $E_{\max }=\left(m_{\mu}{ }^{2}-v^{2}\right) /\left(2 m_{\mu}\right)$.

Radiative corrections to the muon decay spectrum have been calculated only for the case of massless neutrinos. Hence, in fitting with massive neutrinos we used the $V+A$ spectrum without radiative corrections, but made an adjustment (described below) to the fitted value for the $V+A$ decay rate in order to compensate for the missing corrections. The limits on $\mathrm{m}\left(\mathrm{w}_{2}\right)$ that we have computed are therefore only approximate because of the lack of radiative corrections, and also because of several simplifications in the treatment of systematic errors. The limits on $m\left(W_{2}\right)$ are expected to be accurate to $\sim 2 \%$. 
The $V+A$ decay rate without the radiative corrections is plotted in Fig. C. 1 for $\cos \theta=1$ and $m\left(\nu_{\mu R}\right)=0,5,10,15$ and $20 \mathrm{MeV} / \mathrm{c}^{2}$ (solid curves). For comparison, the radiatively corrected $\mathrm{V}+\mathrm{A}$ rate for $m\left(\nu_{\mu R}\right)=0$ and $\cos \theta=1$ is shown by the dashed curve. The ratio of the radiatively corrected $V+A$ rate to the uncorrected rate for $m\left(\nu_{\mu R}\right)=0$ is nearly independent of $\cos \theta$ for $\cos \theta$ near 1 . It decreases from 0.95 at $x=0.97$ to 0.93 at $x=0.99$ approximately 1 inearly; above $x=0.99$ it decreases much more rapidly and approaches 0 as $x \rightarrow 1$.

If neutrinos are massive Majorana particles there are four distinct final states in muon decay (it is assumed that there is no neutrino mixing). If $v_{\mu R}$ is massive but $v_{e R}$ is very $l i g h t$, then the measured spectrum corresponding to the spin-held data will be proportional to the sum

$$
S_{V-A}\left(x, P_{\mu} \cos \theta_{a v}\right)+\varepsilon^{2} S_{V+A, v}\left(x, P_{\mu} \cos \theta_{a v}\right)
$$

where $S_{V-A}\left(x, P_{\mu} \cos \theta_{a v}\right)$ denotes the $V-A$ spectrum for muon polarization equal to $P_{\mu}$ and $\cos \theta=\cos \theta_{a v}, \varepsilon$ is the mass-squared ratio for the gauge bosons $W_{1}$ and $W_{2}$, and $S_{V+A, \nu}\left(x, P_{\mu} \cos \theta_{a v}\right)$ is the $V+A$ spectrum for a massive $\nu_{\mu R}$ with the same normalization as $S_{V-A}\left(x, P_{\mu} \cos \theta_{a V}\right)$. Recall that in the case of massless neutrinos the fitting function for the spin-held data (V.3) was based on the equation (2.2). The sum (C1) can also be written in a form similar to the equation (2.2)

$$
\begin{gathered}
{\left[1-r\left(\theta_{a v}\right)\right] S_{V-A}(x, 1)+r\left(\theta_{a V}\right) S_{V-A}(x, 0)} \\
+\varepsilon^{2}\left\{\left[1-r\left(\theta_{a v}\right)\right] S_{V+A, v}(x, 1)+r\left(\theta_{a V}\right) S_{V+A, v}(x, 0)\right\}
\end{gathered}
$$

where

$$
r\left(\theta_{a v}\right)=1-P_{\mu} \cos \theta_{a v}
$$




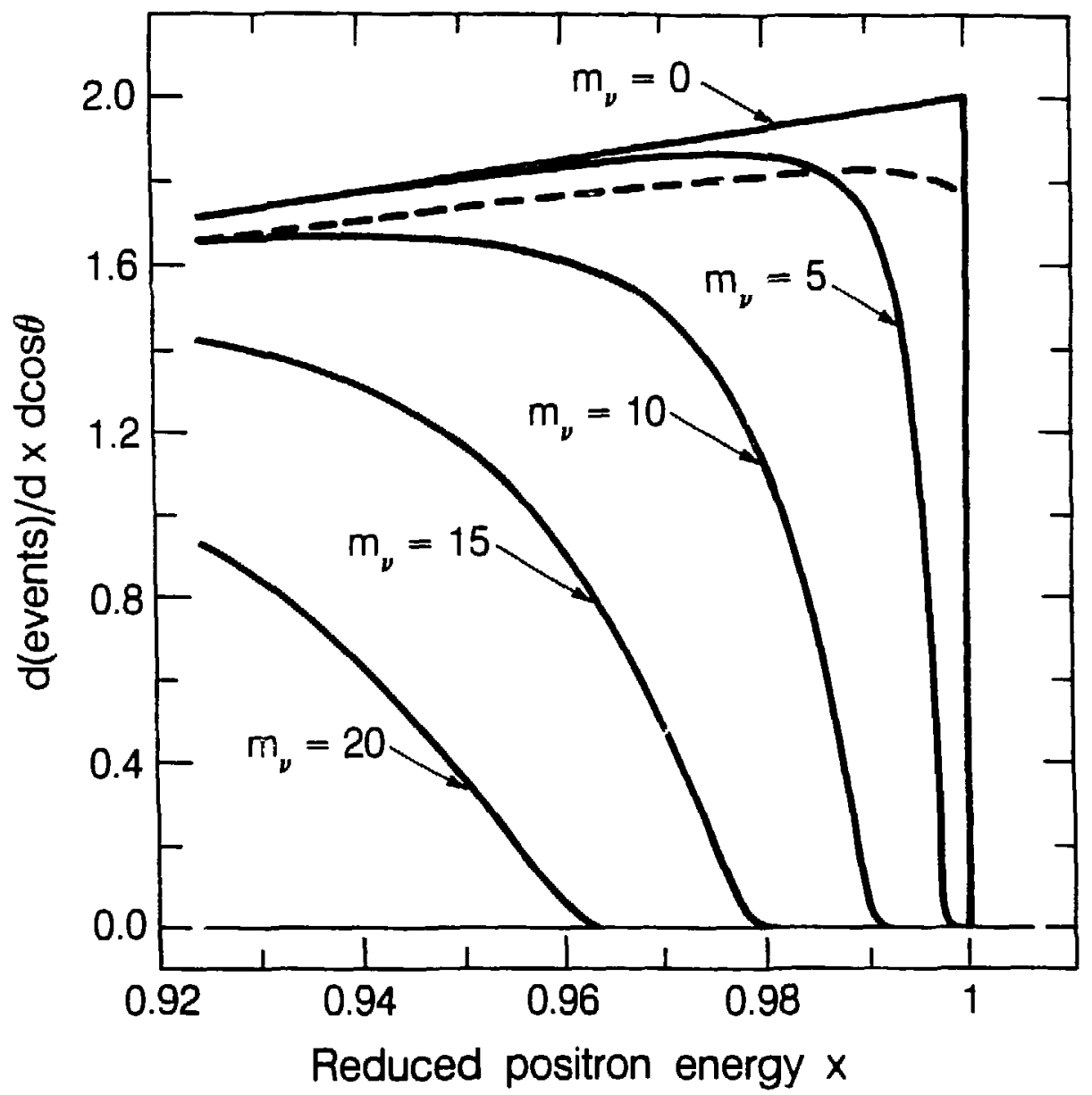

XBL 8512-11760

FIG. C.1. The V+A muon decay rate without radiative corrections for $m\left(v_{\mu}\right)=0,5,10,15$, and $20 \mathrm{MeV} / \mathrm{c}^{2}$ assuming massless $v_{e}$ (solid curves). The dashed curve is the $\mathrm{V}+\mathrm{A}$ radiatively corrected decay rate for $m\left(v_{\mu}\right)=m\left(v_{e}\right)=c$. The decay rates are plotted for the reduced positron energy $x>0.92$. They are normalized so that the integral of the radiatively corrected rate is 1 . 
The fitting procedure for the case of massive neutrinos was therefore very similar to that for massless neutrinos described in section V.A and we will not describe it in detail. The primary difference was that in the fitting function for the spin-held data there was one more free parameter $\varepsilon$, which was constrained to be greater or equal to zero. For the fitting function for the spin-precessed data, it was determined that the contribution of $S_{V+A, v}(x, 0)$ could be ignored because it changed the limit on $\mathrm{m}\left(\mathrm{W}_{2}\right)$ by less than $0.5 \%$.

There was, however, an additional constraint on $\varepsilon$ imposed by the fitted value of the relative rate at the endpoint $r\left(\theta_{a v}\right)$ (i.e. of $\left.1-P_{\mu} \cos \theta_{a v}\right)$ since for a sufficiently light $\nu_{\mu R}$ the polarization of the muon beam delivered to the polarimeter would have been reduced by the effects of the same right-handed currents. The reduction in muon beam polarization due to possible right-handed currents is given by

$$
\Delta P_{\mu}=2 \varepsilon^{2} B_{\nu} F\left(m_{\nu}\right)
$$

where $\beta_{\nu}$ is the velocity of $\nu_{\mu R}$ in $\pi^{+}$decay at rest and $F\left(m_{\nu}\right)$ is the fraction of muons in the beam which were produced in $\pi^{+}$decays mediated by $W_{2}$. The fitted value of $r\left(\theta_{a v}\right)$ was therefore constrained to be

$$
r\left(\theta_{\mathrm{av}}\right) \geq 1-\left(1-\Delta \mathrm{P}_{\mu}\right) \cos \theta_{\mathrm{av}}
$$

with $\Delta \mathrm{P}_{\mu}$ given by $(\mathrm{C} 2)$.

The fraction $F\left(m_{\nu}\right)$, defined just above, decreased as $m\left(\nu_{\mu R}\right)$ increased and as the momentum of the muons, produced in $\pi^{+}$decays mediated by $w_{2}$, apfroached the lower boundary of the beamline momentum bite. For simplicity this fraction was computed assuming a uniform distribution in the $1 \%$ beam momentum bite $\Delta p / p$. The mean 
beam momentum was taken to be $29.45 \mathrm{MeV} / \mathrm{c} . \mathrm{F}\left(\mathrm{m}_{v}\right)$ vanished for $\mathrm{m}\left(v_{\mu \mathrm{R}}\right)>5 \mathrm{MeV} / \mathrm{c}^{2}$

The limits on $E$ [which in turn determine the limits on $m\left(W_{2}\right)$ ] were computed for $m\left(\nu_{\mu R}\right)$ varying from 0 to $16.5 \mathrm{MeV} / \mathrm{c}^{2}$ in steps of $0.5 \mathrm{MeV} / \mathrm{c}^{2}$. For $\mathrm{m}\left(\nu_{\mu \mathrm{R}}\right) \geq 17 \mathrm{MeV} / \mathrm{c}^{2}$ the 1 imits on $\epsilon$ increased dramatically since the endpoint of the $s$ pectrum $S_{V+A, v}\left(x, P_{\mu} \cos \theta_{a V}\right)$ approached the minimum $x=0.97$ used in the fit of the spin-held data. In order to reduce the computational effort only one $f i t$ was made for each value of $m\left(v_{\mu R}\right)$. The $f i t$ was made to the data from all targets in one $\cos \theta_{e}$ bin $\left(\cos \theta_{e} \geq 0.975\right)$. Furthermore, since the systematic error in $\varepsilon$ was different for each value of $m\left(v_{\mu R}\right)$, only the largest systematic errors were considered. For $m\left(\nu_{\mu R}\right)>5 \mathrm{MeV} / \mathrm{c}^{2}$, when there was no constraint on $\varepsilon$ from $r\left(\theta_{a v}\right)\left[r\left(\theta_{a v}\right)=1-P_{\mu} \cos \theta_{a v}\right]$, the systematic error in $\cos \theta_{a y}$ did not propagate into an error in $E$. Hence, the largest systematic errors in $\varepsilon$ for $m\left(\nu_{\mu R}\right)>5 \mathrm{MeV} / \mathrm{c}^{2}$ were due to possible differences in $x=1$ calibration and $x$ acceptance between the spin-held and spin-precessed data sets (see sections VII.A and VII.B). For simplicity, only these two errors were considered also for $m\left(\nu_{\mu R}\right)$ less than $5 \mathrm{MeV} / \mathrm{c}^{2}$.

In order to account for these systematic errors, the limis on $E$ were computed for the four different cases corresponding to $\Delta x_{1}= \pm 0.45 \cdot 10^{-4}$ and $D_{1}= \pm 0.2$, where $\Delta x_{1}$ and $D_{1}$ are defined in sections VII.A and VII.B respectively. of the four limits the most conservative limit on $\varepsilon$ was chosen corresponding to the highest value. For each fit the upper limit on $\varepsilon$ was taken to be the sum of the fitted value, constrained to be greater or equal to zero, plus a 
positive error corresponding to an increase in $x^{2}$ of 1.638 .

$\left[1.638=1.28^{2}\right.$; the probability of an answer lying more than 1.280 above a gaussian with a standard deviation o 19 10\%.]

As mentioned above, the limits on $E$ were computed using the spectrum $S_{V+A, v}\left(x, P_{\mu} \cos \theta_{a v}\right)$ without the radiative corrections. For $m\left(\nu_{\mu R}\right)=0$ the fitted value for $\varepsilon$ was compared to the value found when the radiative corrections were included. Agreement in the two values could be obtained if the former were multiplied by 1.04. Since the effect of the radiative corrections is greatest near the endpoint, one would expect that the adjustment in the fitted value for $\varepsilon$ should be smaller for $m\left(\nu_{\mu R}\right)>0$, because the spectrum for $m\left(\nu_{\mu R}\right)>0$ rises more slowly near the endpoint (see Fig. C.1). Conservatively, however, the limits on $\varepsilon$ were multiplied by 1.04 for all values of $m\left(\nu_{\mu R}\right)$. The approximate limits on $m\left(W_{2}\right)$ versus $m\left(\nu_{\mu R}\right)$, computed from the limits on $\varepsilon$ assuming $m\left(W_{1}\right)=81 \mathrm{GeV} / \mathrm{c}^{2}$, are shown in Fig. VIII. 1 in section VIII.F. These limits were commented upon in the last paragraph of that section. 


\section{REFERENCES}

1 S. Glashow, Nucl. Phys. 22, 529 (1961); S. Weinberg, Phys. Rev. Lett. 19, 1264 (1967); A. Salam, in Elementary Particle Theory, ed. N. Svastholm (Stockholm, 1968)

2 K. Mursula, M. Roos, and F. Scheck, Nucl. Phys. B219, 321 (1983);

K. Mursula and F. Scheck, Nucl. Phys. B253, 189 (1985).

3 J.C. Pati and A. Salam, Phys. Rev. D10, 275 (1974);

R.N. Mohapatra and J.C. Pat1, Phys. Rev. D11, 566, 2558 (1974);

G. Senjanovic and R.N. Mohapatra, Phys. Rev. D12, 1502 (1975).

4 G. Senjanovic, Nucl. Phys. B135, 334 (1979).

5 M. Gell-Mann, P. Ramond, and R. Slansky (unpublished);

R. N. Mohapatra and G. Senjanovic, Phys. Rev. D23, 165 (1981).

6 G. Beall, M. Bander, and A. Son1, Phys. Rev. Lett. 4 8, 848 (1982);

R. N. Mohapatra, G. Senjanovic, and M. D. Tran, Phys. Rev. D28, 546 (1983); F.J. Gilman and M.H. Reno, Phys. Lett. 127B, 426 (1983).

7. J. Donahue and B. Holstein, Phys. Lett. 113B, 382 (1982). See also I.I. Bigi and J.M. Frere, Phys. Lett. 110B, 255 (1982).

- F.I. Olness and M.E. Ebel, Phys. Rev. D30, 1034 (1984).

$9 \quad \mathrm{i}$ : Wolfenstein, Phys. Rev. D29, 2130 (1984)

10 M.A.B. Beg, R.V. Budny, R. Mohapatra, and A. Sirlin, Phys. Rev. Lett. 48,1252 (1977); B.R. Holstein and S.B. Treiman, Phys. Rev. D16, 2369 (1977).

11 D.P. Stoker, et al., Phys. Rev. Lett. 54, 1887 (1985).

12 D.P. Stoker, Ph. D. Thesis, LBL report LBL-20324 (1985). 
13 The primary input to the world average is V.V. Akhmanov, et al., Yad. Fiz. 6, $316(1967)$

14 The primary input to the world average is J. Peoples, Nevis Cyclotron Report No. 147 (1966) (unpublished).

is J. Van Klinken, Nucl. Phys. 75, 145 (1966)

16 J. Van Klinken, et al., Phys. Rev. Lett. 50, 94 (1983)

1) D. Schreiber and F.T. Calaprice, private communication;

D. Schreiber, Ph.D. Thesis, Princeton University, 1983 (unpublished). We calculated the contour plotted in Fig. I.1 using $A(0)=.0363 \pm .0008$; ft ratio $=1.797 \pm .002$. See also F.T. Calaprice, S.J. Freedman, W.C. Mead, and H.C. Vantine, Phys. Rev. Lett. 35, 1566 (1975).

1o H. Abramowicz, et al., Z. Phys. C12, 225 (1982).

19 T.D. Lee and C.N. Yang, Phys. Rev. 108, 1611 (1957).

20 F. Scheck, Phys. Lett. $44 \mathrm{C}, 187$ (1978).

21 C.G. Wohl, et al. (Particle Data Group), Rev. Mod. Phys. 56, S1 (1984). Primary input to $p$ is Ref. 14; primary input to $n$ is F. Corriveau et al., Phys. Lett. 129B, 260 (1983) and S.E. Derenzo, Phys. Rev. 181, 1854 (1969); primary input to $\xi P_{\mu}$ is Ref. 13; primary input to $\delta$ is D. Fryberger, Phys. Rev. 166, 1379 (1968).

22 Radiative corrections in muon decay are reviewed by A.M. Sachs and A. Sirlin, in Muon Physics, Vol. II, V. Hughes and C.S. Wu, eds. (Academic Press, New York, 1975). 23 M.A.B. Beg and A. Sirlin, Ann. Rev. Nucl. Sci. 24, 579 (1974). 
24 V. Fluorescu and 0. Kamei, Nuovo Cimento 61A, 967 (1968).

25 A.E. Pifer, T. Bowen, and K.R. Kendall, Nucl. Inst. Meth. 135, $39(1976)$.

26 C.J. Oram, J.B. Warren, G.M. Marshall, and J. Doornbos, Nucl. Inst. Meth. 179, 95 (1981).

27 The deceleration and thermalization of muons in matter has been reviewed by J.H. Brewer, K.M. Crowe, F.N. Gygax, and A. Schenck, in Muon Physics, Vol. III, Y. Hughes and C.S. Wu, eds. (Academic Press, New Ycrk, 1975).

28 G.W. Ford and C.J. Mullin, Phys. Rev. 108, 477 (1957).

29 V. Hughes, Phys. Rev. 108, 1106 (1957).

so R. Sagane, W. Dudziak, and J. Vedder, Phys. Rev. 95, 863 (1954).

31 K. Halbach, private communication. The mathematical formal ism of first-order optics approximation for cylindrically symmetric field is outlined In Appendix A of Ref. 12 .

32 B.P. Nigam, M.K. Sundaresan, and T.A. Wu, Phys. Rev. 115, 491 (1959); J.B. Marion and B.A. Zimmerman, Nucl. Instr. Meth. 51, 93 (1967).

33 P. Herczeg, LANL preprint LA-UR-85-2761.

34 M. Abolins et al., in Proc. 1982 DPF Summer Study on Elem. Part. Phys. and Future Facilities, p. 274, R. Donaldson, R. Gustafson, and F. Palge, eds. (Snowmass, 1982); S. Yamada, in Proc. XXII Int. Conf. on High Energy Physics, p. 72, A. Meyer and E. Wieczorek, eds. (Lelpzig, 1984); M. Althoff, et al., Z. Phys. C22, 13 (1984). 35 M.E. Peskin, private communication. 
36 K.D. Lane and E. Barany, private communication.

37 F. Wilczek, Phys. Rev. Lett. 49, 1549 (1982).

з в E. Eichten, I. Hinchliffe, K. Lane, and C. Quigg, Rev. Mod. Phys. 56, 579 (1984).

39 R.E. Shrock, Phys. Rev. D24, 1275 (1981). 
This report was done with support from the Department of Ener 5 . Any conclusions or opinions expresed in this report represent solety thoue of the author(b) and not acossiarily those of The Regents of the Univeraity of California, the Lawrence Bertazley Laboratory or the Department of Enerzy.

Reference to a company or product mame does not imply epproval or recommendation of the product by the University of Califomin or the U.S. Department of Energy to the exclusion of others that may be suitable. 